\title{
A nonhydrostatic, isopycnal-coordinate ocean model for internal waves
}

\author{
Sean Vitousek ${ }^{\mathrm{a}, *}$, Oliver B. Fringer ${ }^{\mathrm{a}}$ \\ ${ }^{a}$ Environmental Fluid Mechanics Laboratory, Stanford University, Stanford, CA, 94305
}

Abstract

We present a nonhydrostatic ocean model with an isopycnal (densityfollowing) vertical coordinate system. The primary motivation for the model is the proper treatment of nonhydrostatic dispersion and the formation of nonlinear internal solitary waves. The nonhydrostatic, isopycnal-coordinate formulation may be preferable to nonhydrostatic formulations in $z$ - and $\sigma$ coordinates because it improves computational efficiency by reducing the number of vertical grid points and eliminates spurious diapycnal mixing and solitary-wave amplitude loss due to numerical diffusion of scalars. The model equations invoke a mild isopycnal-slope approximation to remove small metric terms associated with diffusion and nonhydrostatic pressure from the momentum equations and to reduce the pressure Poisson equation to a symmetric linear system. Avoiding this approximation requires a costlier inversion of a non-symmetric linear system. We demonstrate that the model is capable of simulating nonlinear internal solitary waves for simplified and

\footnotetext{
${ }^{*}$ Corresponding author

Email addresses: seanv@stanford.edu (Sean Vitousek), fringer@stanford.edu (Oliver B. Fringer)

Preprint submitted to Ocean Modelling

August 22, 2014
}

(C) 2014. This manuscript version is made available under the Elsevier user license http://www.elsevier.com/open-access/userlicense/1.0/ 
physically-realistic ocean-scale problems with a reduced number of layers. Keywords: nonhydrostatic model, isopycnal coordinates, multi-layer model, internal waves, solitary waves, ocean modeling

\section{1. Introduction}

\section{1.1. Literature review}

3 Simulating internal waves is one of the most computationally challenging 4 tasks in ocean modeling. Large-scale processes such as internal tides can be 5 modeled reasonably well with computationally-inexpensive hydrostatic mod6 els (Kantha and Clayson, 2000). Simulations of nonlinear internal solitary 7 waves, on the other hand, require computationally-expensive nonhydrostatic 8 models to represent dispersive behavior. Nonhydrostatic models can incur 9 an order of magnitude increase in computational time relative to hydro10 static models due to the elliptic solver for the nonhydrostatic or dynamic 11 pressure (Fringer et al., 2006). Furthermore, simulations of nonlinear internal solitary waves require high horizontal grid resolution to ensure that numerically-induced dispersion ${ }^{1}$ is small relative to physical dispersion (Vitousek and Fringer, 2011).

The vertical coordinate system is often reported as the most important aspect in the design of an ocean model (Griffies et al., 2000; Chassignet et al., 2000; Willebrand et al., 2001; Chassignet, 2011). The three vertical coordinate systems typically used in ocean models are: (1) Height or

\footnotetext{
${ }^{1}$ from truncation error of the discretized model equations
} 
$z$-coordinates, (2) Terrain-following or $\sigma$-coordinates, and (3) Isopycnal or $\rho$-coordinates. Each approach has numerous advantages and disadvantages as outlined in Griffies et al. (2000). Existing nonhydrostatic models employ $z$ - or $\sigma$-coordinates [e.g. (Mahadevan et al., 1996a,b), MITgcm (Marshall et al., 1997b,a), SUNTANS (Fringer et al., 2006) for $z$-coordinates, POM (Kanarska and Maderich, 2003), BOM (Heggelund et al., 2004), ROMS (Kanarska et al., 2007), FVCOM (Lai et al., 2010a) for $\sigma$-coordinates and ICOM (Ford et al., 2004a,b) for vertically unstructured coordinates]. $z$ - and $\sigma$ coordinate models are capable of representing overturning motions and eddies (e.g. Kelvin-Helmholtz, Rayleigh-Taylor, and other instabilities) that are associated with many small-scale nonhydrostatic processes. Isopycnalcoordinate models, on the other hand, cannot represent overturning motions or unstable stratification. This deficiency leads to the notion that isopycnal coordinates are not suitable for modeling nonhydrostatic processes (Adcroft and Hallberg, 2006). Consequently, existing isopycnal models such as MICOM/HYCOM (Bleck et al., 1992; Bleck, 2002), HIM (Hallberg, 1995, 1997; Hallberg and Rhines, 1996), POSEIDON (Schopf and Loughe, 1995), POSUM (Higdon and de Szoeke, 1997; de Szoeke, 2000) so far exclusively employ the hydrostatic approximation. While clearly a deficiency of isopycnal models, the inability to represent unstable stratification is an issue for hydrostatic and nonhydrostatic isopycnal formulations alike. In this paper, we do not propose a means for isopycnal-coordinate models to represent unstable stratification - this task is clearly suited to $z$ - and $\sigma$-coordinate models. 
Instead, the primary motivation behind the model presented in this paper is the proper treatment of dispersion and the formation of nonlinear internal solitary waves in the context of an isopycnal-coordinate model. Internal solitary waves are clearly nonhydrostatic and not associated with overturning motions. Although overturning structures may exist in the vicinity of internal wave generation sites that might preclude the use of an isopycnal-coordinate model, a model is not required to resolve these structures to obtain a good prediction of the internal wave generation. For example, Klymak and Legg (2010) and Klymak et al. (2010) developed a simple scheme that faithfully captures dissipation and mixing related to internal wave generation at a ridge and show that their hydrostatic model was almost identical to the nonhydrostatic model in predicting the generation dynamics. This suggests that, while small-scale, nonhydrostatic processes related to internal wave generation are indeed complex, they can be parameterized appropriately in large-scale $z$-, $\sigma-$, and isopycnal-coordinate models that do not resolve them. Hence, a nonhydrostatic, isopycnal-coordinate formulation may be suitable for modeling internal or interfacial waves.

In the context of internal wave modeling, isopycnal-coordinates may provide some advantages over $z$ - and $\sigma$-coordinates. Isopycnal or density-following coordinates provide natural representations of (stably) stratified fluids. This reduces the number of vertical grid points from $\mathcal{O}(100)$ in $z$ - and $\sigma$-coordinate models to $\mathcal{O}(1)$ in isopycnal coordinates (Bleck and Boudra, 1981). Ideally, in locations where the internal wave structure is predominantly mode-1, an 
isopycnal model with only two layers may suffice (Simmons et al., 2011). The primary disadvantage of modeling nonhydrostatic pressure is that it requires solution of a three-dimensional elliptic (Poisson) equation for the nonhydrostatic pressure which significantly increases the computational cost relative to the hydrostatic model. Solving this elliptic equation requires optimally $\mathcal{O}(N)$ operations (Briggs et al., 2000) where $N$ is the number of grid cells. Isopycnal coordinates can improve the efficiency of nonhydrostatic methods by reducing the required number of vertical grid points by an order of magnitude relative to $z$ - and $\sigma$-coordinate models. Thus, reducing the number of vertical layers and thus the overall number of grid cells by an order of magnitude can result in at least one order of magnitude reduction in computational cost.

Another advantage of isopycnal-coordinates is the reduction or elimination of spurious diapycnal mixing. Transport in the ocean predominantly occurs along rather than across isopycnal surfaces (Iselin, 1939; Montgomery, 1940). In many applications, spurious diapycnal mixing, arising from numericallydiffusive truncation error in scalar transport schemes in $z$ - and $\sigma$-coordinate models (Fringer et al., 2005), can be larger than physical diapycnal mixing (Griffies et al., 2000). Isopycnal coordinates, on the other hand, are not susceptible to spurious diapycnal mixing because the governing equations are constructed to directly control the amount of diapycnal transport - if any (Bleck and Boudra, 1981; Griffies et al., 2000). Hence, the problem of energy loss due to spurious diapycnal mixing that occurs in numerical models dur- 
ing the formation and propagation of internal solitary waves (Hodges et al., 2006) may be reduced or eliminated with isopycnal coordinates.

\subsection{Outline of the proposed model}

Existing approaches for simulating nonhydrostatic internal waves include $z$ - and $\sigma$-coordinate models applied at high resolution in 3-D (Fringer et al., 2006; Vlasenko and Stashchuk, 2007; Vlasenko et al., 2009, 2010; Lai et al., 2010b; Zhang et al., 2011; Guo et al., 2011) or 2-D slices (Scotti et al., 2007, 2008; Buijsman et al., 2010) or asymptotic/Boussinesq-type approaches using 2-layer (Brandt et al., 1997; Choi and Camassa, 1999; Lynett and Liu, 2002; de la Fuente et al., 2008; Steinmoeller et al., 2012) or multi-layer models (Liu and Wang, 2012). Asymptotic or Boussinesq-type approaches do not require a pressure projection method. Instead, they include higher-order derivatives to account for the nonlinear and dispersive behavior. Boussinesq-type models have a limited range of applicability that is often the weakly nonlinear, weakly nonhydrostatic regime. To extend this range of applicability, more terms may be included or advanced formulations may be introduced. However, this can lead to an unwieldy set of governing equations containing high-order, mixed time-and-space derivatives.

The formulation presented here is intended to be flexible (using an arbitrary number of layers) and straightforward (resembling existing ocean models). The numerical method uses a pressure projection method which results in an elliptic equation for the dynamic pressure (as in $z$-and $\sigma$-coordinate 
models). The elliptic equation in isopycnal coordinates results in a nonsymmetric system of linear equations. However, by invoking a mild-slope approximation, the system becomes symmetric and remarkably similar to the elliptic equation in $z$-coordinates.

Another significant difference between existing isopycnal models and the model presented here (besides the treatment of nonhydrostatic pressure) is the time-stepping procedure. Most isopycnal models use mode-splitting to treat fast free-surface gravity waves (Bleck and Smith, 1990; Higdon and Bennett, 1996; Higdon and de Szoeke, 1997; Hallberg, 1997). The current model uses an implicit time-stepping procedure for the free surface following Casulli (1999) which is common in nonhydrostatic models in $z$ - and $\sigma$-coordinates (a list of nonhydrostatic models using implicit time-stepping procedures is given in Vitousek and Fringer (2013)). Casulli (1997) developed a hydrostatic, isopycnal model with an implicit time-stepping procedure for the free surface and layer heights. In his approach, the gradient of the Montgomery potential $(M)$, which represents the hydrostatic pressure in isopycnal coordinates, is discretized implicitly. Thus, computing the free-surface and interface heights requires the inversion of a large (3-D) system of equations which is comparable in cost to the solution of the (3-D) elliptic equation for the nonhydrostatic pressure. The current model is similar to the approach of Casulli (1997). However, we split the Montgomery potential into barotropic 
131

$\left(M^{(\mathrm{bt})}\right)$ and baroclinic $\left(M^{(\mathrm{bc})}\right)$ parts according to

$$
M=\rho_{0}^{-1}\left(p_{h}+\rho g z\right)=\underbrace{\rho_{0}^{-1}\left(p_{s}+\rho_{0} g \eta\right)}_{=M^{(\mathrm{bt})}}+M^{(\mathrm{bc})},
$$

where $\rho$ is the density ( $\rho_{0}$ is the reference density), $p_{h}$ is the hydrostatic pressure, $p_{s}$ is the surface (atmospheric) pressure, $\eta$ is the free-surface height, $z$ is the interface location, and the term $\rho g z$ originates from the transformation to isopycnal coordinates. Because the barotropic and baroclinic portions of the Montgomery potential represent fast free-surface and slow internal-gravity waves, they are discretized implicitly and explicitly, respectively. This discretization requires inversion of a 2 -D system in the horizontal to compute the free-surface height as is the case with implicit free-surface models in $z$ - and $\sigma$-coordinates. The computational cost of the 2-D inversion for the free-surface height is minimal compared to the 3-D inversion for the nonhydrostatic pressure.

\subsection{Use of Lagrangian coordinates for the nonhydrostatic equations}

Adcroft and Hallberg (2006) conclude that the nonhydrostatic projection method and use of Lagrangian vertical coordinates are mutually exclusive. Their argument is based on how the Lagrangian algorithm prescribes the material derivative of the (general) vertical coordinate, $\dot{r}$, in the continuity equation while in the nonhydrostatic projection method this term should instead come from the vertical momentum equation. This leads to a conundrum in which one cannot simultaneously supply and diagnose a quantity 
in an equation (Adcroft and Hallberg, 2006). The present study does not seem limited by this issue. When using pure isopycnal coordinates, $r \equiv \rho$ and $\dot{r} \equiv \dot{\rho} \equiv \frac{D \rho}{D t} \equiv 0$ (since density is treated as a materially conserved quantity). Consequently, $\dot{r} \equiv 0$ is prescribed in the continuity equation and this leads to an evolution equation for the isopycnal layer heights that is consistent with the material conservation of density. After the layer heights are evolved, the projection method uses the vertical velocity from the vertical momentum equation in the divergence-free from of the continuity equation. We note that the two forms of the continuity equation, namely the layerheight equation and the divergence-free continuity equation, are, in fact, the same equation and the condition $\dot{r} \equiv 0$ gives rise to the layer-height equation. After the nonhydrostatic projection step, a final vertical velocity is computed using the divergence-free form of the continuity equation. Our interpretation of the Adcroft and Hallberg (2006) conundrum is that it implies that the vertical velocity computed with the projection step cannot be consistent with that which is implied by the material conservation of density in the layer-height equation. This does not appear to be an issue in our approach nor in any of the commonly used nonhydrostatic methods, likely because maintaining absolute consistency is unnecessary, but instead must be maintained to some order of accuracy. Specifically, when the free surface is evolved in typical nonhydrostatic models, this implies a vertical velocity from a discrete kinematic condition which may not necessarily be the same as that which is computed with the projection method. As discussed in Section 
3.3, the popular approach of evolving the free-surface using the hydrostatic provisional velocities rather than the final nonhydrostatic velocities still results in a method that is second-order accurate (Kang and Fringer, 2005; Vitousek and Fringer, 2013). This suggests that the effective vertical velocity arising from applying the kinematic condition to the continuity equation is relatively unimportant. Further support for the method developed here comes from the fact that the numerical algorithm (presented in Section 3) simplifies to popular methods used in nonhydrostatic $z$-coordinate models with a free surface (e.g. Fringer et al. (2006)) in the case that only one vertical layer is used. Recently, Klingbeil and Burchard (2013) extended a generalized vertical-coordinate model (GETM) to include the nonhydrostatic pressure without using the projection method, which improves performance owing to the lack of an elliptic equation for the nonhydrostatic pressure. Like our method, the approach of Klingbeil and Burchard (2013) does not suffer from the conundrum of Adcroft and Hallberg (2006). Therefore, we do not suggest that our method or that of Klingbeil and Burchard (2013) present solutions to the conundrum, but instead that its potential limitations can be circumvented.

\subsection{Synopsis}

The remainder of this paper is divided into five sections. Section 2 presents the derivation of the governing equations in continuous isopycnal coordinates. Section 3 presents the numerical method used to solve the gov- 
erning equations. Section 4 discusses stability and convergence of the model. Section 5 presents model validation with several idealized test cases. Finally, Section 6 presents the conclusions. Lengthy derivations relevant to the model formulation are presented in appendices.

\section{Governing equations}

\subsection{Equations in Cartesian coordinates}

The governing equations are the Reynolds-Averaged Navier-Stokes (RANS) equations with the Boussinesq approximation in a rotating reference frame. In Cartesian coordinates, the equations are given by

$\frac{\partial u}{\partial t}+\nabla \cdot(\mathbf{u} u)-f v+\tilde{f} w=-\frac{1}{\rho_{0}} \frac{\partial p_{h}}{\partial x}-\frac{\partial q}{\partial x}+\nabla_{H} \cdot\left(\nu_{H} \nabla_{H} u\right)+\frac{\partial}{\partial z}\left(\nu_{v} \frac{\partial u}{\partial z}\right)$,

$\frac{\partial v}{\partial t}+\nabla \cdot(\mathbf{u} v)+f u \quad=-\frac{1}{\rho_{0}} \frac{\partial p_{h}}{\partial y}-\frac{\partial q}{\partial y}+\nabla_{H} \cdot\left(\nu_{H} \nabla_{H} v\right)+\frac{\partial}{\partial z}\left(\nu_{v} \frac{\partial v}{\partial z}\right)$

$\frac{\partial w}{\partial t}+\nabla \cdot(\mathbf{u} w)-\tilde{f} u \quad=\quad-\frac{\partial q}{\partial z}+\nabla_{H} \cdot\left(\nu_{H} \nabla_{H} w\right)+\frac{\partial}{\partial z}\left(\nu_{v} \frac{\partial w}{\partial z}\right)$,

where $\mathbf{u}=[u, v, w]^{T}$ is the velocity vector, $f=2 \Omega \sin (\phi)$ is the traditional Coriolis parameter and $\tilde{f}=2 \Omega \cos (\phi)$ is the nontraditional Coriolis parameter (see e.g. White and Bromley (1995); Marshall et al. (1997b); Gerkema 
et al. (2008)), where $\phi$ is the latitude and $\Omega$ is the Earth's angular velocity, $p_{h}(z)=p_{s}+\rho_{0} g(\eta-z)+g \int_{z}^{\eta}\left(\rho-\rho_{0}\right) d z$ is the hydrostatic pressure (derived from integrating the hydrostatic balance $\frac{\partial p_{h}}{\partial z}=-\rho g$ ), where $p_{s}$ is the atmospheric pressure at the surface, $g$ is gravity, $\eta$ is the free-surface height, $\rho$ is the density and $\rho_{0}$ is the (constant) reference density, and $\nu_{H}$ and $\nu_{v}$ are the horizontal and vertical eddy-viscosities, respectively. The difference between the nonhydrostatic equation set and the hydrostatic equation set is the treatment of the full vertical momentum balance (2c) and the presence of the nonhydrostatic pressure terms involving $q$ on the right-hand side (RHS) of the momentum equations (2). The kinematic nonhydrostatic pressure is given by $q=p_{\mathrm{nh}} / \rho_{0}$ in the notation following Casulli (1999), where $p_{\mathrm{nh}}$ is the nonhydrostatic or dynamic pressure due to the vertical momentum or acceleration of the fluid.

Equations (2) are subject to the incompressibility constraint

$$
\nabla \cdot \mathbf{u}=0
$$

and conservation of density

$$
\frac{D \rho}{D t}=\frac{\partial \rho}{\partial t}+\nabla \cdot(\mathbf{u} \rho)=0
$$

where we ignore diffusion of density although it can be added following the methods in standard isopycnal models (Hallberg, 2000). Diffusion of density leads to $\dot{\rho}=\frac{D \rho}{D t} \neq 0$ and to the conundrum of Adcroft and Hallberg (2006) 
related to the treatment of nonhydrostatic pressure. We present possible solutions to this issue in Section 3.3.

\subsection{Mild-slope equations in isopycnal coordinates}

The momentum equations in isopycnal coordinates result from applying the coordinate transformation

$$
\left[\begin{array}{c}
\frac{\partial}{\partial x} \\
\frac{\partial}{\partial y} \\
\frac{\partial}{\partial z} \\
\frac{\partial}{\partial t}
\end{array}\right]=\left[\begin{array}{cccc}
1 & 0 & -\frac{z_{x^{*}}}{z_{\rho}} & 0 \\
0 & 1 & -\frac{z_{y^{*}}}{z_{\rho}} & 0 \\
0 & 0 & \frac{1}{z_{\rho}} & 0 \\
0 & 0 & -\frac{z_{t^{*}}}{z_{\rho}} & 1
\end{array}\right]\left[\begin{array}{c}
\frac{\partial}{\partial x^{*}} \\
\frac{\partial}{\partial y^{*}} \\
\frac{\partial}{\partial \rho} \\
\frac{\partial}{\partial t^{*}}
\end{array}\right],
$$

(see e.g. Müller (2006)) to the momentum equations in Cartesian coordinates (2). Next, as demonstrated in Appendix A, the resulting equations are subjected to an mild isopycnal-slope approximation to remove small metric terms associated with diffusion and the nonhydrostatic pressure from the momentum equations. Neglecting the small metric terms associated with diffusion based on a mild isopycnal-slope approximation is perhaps unnecessary. Solomon (1971) and Redi (1982) argued that the representation of diffusion in an isopycnal coordinate system (i.e. using the diffusion operators in the equations below without the transformation metric terms) is preferable to the representation in a Cartesian coordinate system in order to faithfully capture the tendency for along-isopycnal mixing of scalars and momentum 


$$
\begin{aligned}
\frac{\partial u}{\partial t}+\mathbf{u}_{H} \cdot \nabla_{H} u-f v+\tilde{f} w & =-\frac{\partial M}{\partial x}-\frac{\partial q}{\partial x}+\nabla_{H} \cdot\left(\nu_{H} \nabla_{H} u\right)+\frac{1}{z_{\rho}} \frac{\partial}{\partial \rho}\left(\nu_{v} \frac{1}{z_{\rho}} \frac{\partial u}{\partial \rho}\right) \\
\frac{\partial v}{\partial t}+\mathbf{u}_{H} \cdot \nabla_{H} v+f u & =-\frac{\partial M}{\partial y}-\frac{\partial q}{\partial y}+\nabla_{H} \cdot\left(\nu_{H} \nabla_{H} v\right)+\frac{1}{z_{\rho}} \frac{\partial}{\partial \rho}\left(\nu_{v} \frac{1}{z_{\rho}} \frac{\partial v}{\partial \rho}\right) \\
\frac{\partial w}{\partial t}+\mathbf{u}_{H} \cdot \nabla_{H} w-\tilde{f} u & =-\frac{1}{z_{\rho}} \frac{\partial q}{\partial \rho}+\nabla_{H} \cdot\left(\nu_{H} \nabla_{H} w\right)+\frac{1}{z_{\rho}} \frac{\partial}{\partial \rho}\left(\nu_{v} \frac{1}{z_{\rho}} \frac{\partial w}{\partial \rho}\right)
\end{aligned}
$$

where the star $\left(^{*}\right)$ notation has been dropped for convenience and $M$ is the Montgomery Potential given in Equation (1). The main difference between the equations in Cartesian coordinates (2) and isopycnal coordinates (6), besides the appearance of the Montgomery potential, is the absence of vertical advection of momentum terms in Equations (6).

The continuity equation (3) can be written in two different ways. In existing isopycnal models, the continuity equation is often written as

$$
\frac{\partial h}{\partial t}=-\nabla_{H} \cdot\left(\mathbf{u}_{H} h\right)
$$

251 where $h=-\Delta \rho \frac{\partial z}{\partial \rho}=-\Delta \rho z_{\rho}$ is the thickness of an isopycnal layer. Alterna- 
tively, the divergence-free form of the continuity equation is written as

$$
\frac{\partial}{\partial x}\left(z_{\rho} u\right)+\frac{\partial}{\partial y}\left(z_{\rho} v\right)+\frac{\partial w}{\partial \rho}=0
$$

We note that Equation (7) is used to evolve the thickness of isopycnal layers and Equation (8) is used to calculate the nonhydrostatic pressure via an elliptic equation that enforces continuity. This procedure is similar to methods that are typically employed in nonhydrostatic, $z$ - and $\sigma$-coordinate models with a free surface and is discussed in Section 3. The continuity equation (8) is also subject to the mild-slope approximation as discussed in Appendix A. By invoking this approximation, the discrete elliptic equation for the nonhydrostatic pressure reduces to a symmetric, 5-diagonal linear system in 2-D (7-diagonal in 3-D) as demonstrated in Appendix E. Berntsen and Furnes (2005) invoked a similar approximation by neglecting metric terms appearing from the transformation to $\sigma$-coordinates. Their approximation also results in a 5-diagonal linear system of equations in 2-D for the nonhydrostatic pressure and produces simulation results that are nearly identical to the full (unapproximated) elliptic equation (Keilegavlen and Berntsen, 2009).

\section{Numerical Method}

The numerical method used to solve the governing equations in isopycnal coordinates must be capable of handling three specific numerical challenges: [1] treatment of stiffness caused by fast free-surface gravity waves, [2] wetting 
and drying of isopycnal layers, [3] solution of the nonhydrostatic pressure. As discussed in Section 3.1, to treat fast free-surface gravity waves, we split the barotropic and baroclinic parts of the Montgomery potential and discretize them implicitly and explicitly, respectively. Drying of isopycnal layers is handled by using upwind or MPDATA schemes to evolve the layer heights as discussed in Section 3.2. Finally, the solution procedure for the nonhydrostatic pressure in isopycnal coordinates, as discussed in Section 3.3, is similar to procedures in z-coordinates (such as Casulli (1999); Fringer et al. (2006)). For clarity, we present the numerical formulation in $2-\mathrm{D}$ (in an $x$ - $z$ plane). The method is easily extended to $3-\mathrm{D}$.

\subsection{Discretized momentum equations}

The momentum equations (6) are discretized using a second-order accurate finite-difference method on a staggered C-grid in which the velocities are defined at cell faces and quantities such as the layer heights and nonhydrostatic pressure are defined at cell centers. The model setup and location of the variables of interest are shown on Figure 1. In order to discretize the governing equations in continuous isopycnal coordinates (6) and obtain the model equations with a discrete number of layers, we have used a secondorder accurate finite-difference approximation given by

$$
\frac{1}{z_{\rho}} \frac{\partial \phi}{\partial \rho} \approx \frac{\phi_{k-1 / 2}-\phi_{k+1 / 2}}{h_{k}}
$$




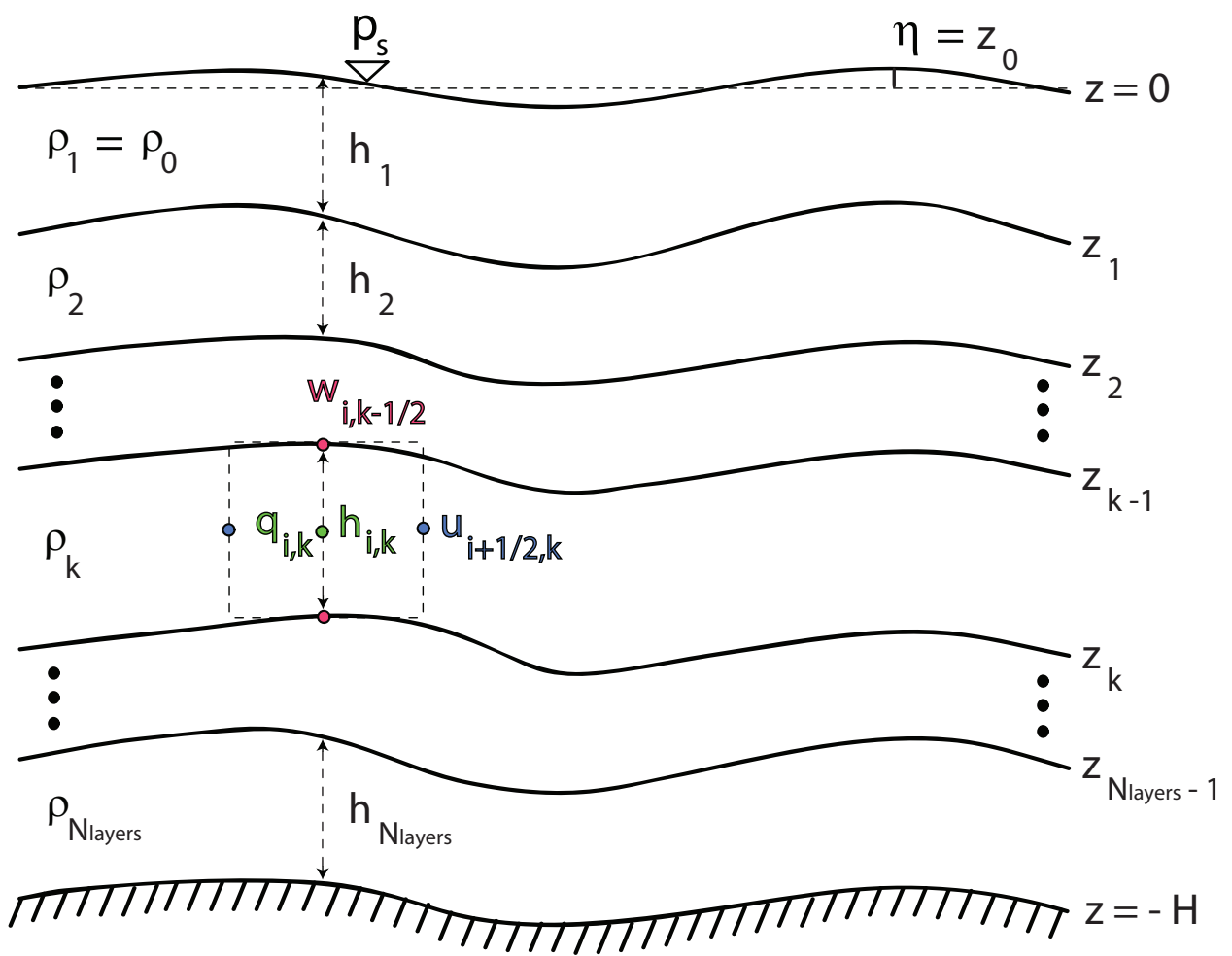

Figure 1: The isopycnal model setup.

The layer index, $k$, increases downward as is conventional in isopycnal models (Hallberg, 1997; Bleck, 2002). Use of Equation (9) for the vertical derivatives and an evolution equation for the layer height $h_{k}$ makes the numerical method a LVD algorithm (Adcroft and Hallberg, 2006; Hallberg and Adcroft, 2009) and also allows simulation of fluids with constant density.

Using the spatial discretization above and following existing nonhydrostatic operator-splitting procedures, the discrete momentum equations become 


$$
\begin{aligned}
\frac{u_{i+\frac{1}{2}, k}^{*}-u_{i+\frac{1}{2}, k}^{n}}{\Delta t} & =-g \frac{\eta_{i+1}^{(n+1)_{\operatorname{Im}}}-\eta_{i}^{(n+1)_{\operatorname{Im}}}}{\Delta x} \\
& +F_{i+\frac{1}{2}, k}^{(n)_{\operatorname{Ex}}}+\frac{\left(\tau^{x}\right)_{i+\frac{1}{2}, k-\frac{1}{2}}^{\left(n+\theta^{*}\right)}-\left(\tau^{x}\right)_{i+\frac{1}{2}, k+\frac{1}{2}}^{\left(n+\theta^{*}\right)}}{h_{i+\frac{1}{2}, k}^{n}}-\alpha \frac{q_{i+1, k}^{n-\frac{1}{2}}-q_{i, k}^{n-\frac{1}{2}}}{\Delta x} \\
\frac{w_{i, k+\frac{1}{2}}^{*}-w_{i, k+\frac{1}{2}}^{n}}{\Delta t} & =G_{i, k+\frac{1}{2}}^{(n)_{\operatorname{Ex}}}+\frac{\left(\tau^{z}\right)_{i, k}^{\left(n+\theta^{*}\right)}-\left(\tau^{z}\right)_{i, k+1}^{\left(n+\theta^{*}\right)}}{h_{i, k+\frac{1}{2}}^{n}}-\alpha \frac{q_{i, k}^{n-\frac{1}{2}}-q_{i, k+1}^{n-\frac{1}{2}}}{h_{i, k+\frac{1}{2}}^{n}}
\end{aligned}
$$

representing the hydrostatic prediction step, and

$$
\begin{aligned}
& \frac{u_{i+\frac{1}{2}, k}^{n+1}-u_{i+\frac{1}{2}, k}^{*}}{\Delta t}=-\frac{\left(q_{c}\right)_{i+1, k}^{n+\frac{1}{2}}-\left(q_{c}\right)_{i, k}^{n+\frac{1}{2}}}{\Delta x} \\
& \frac{w_{i, k+\frac{1}{2}}^{n+1}-w_{i, k+\frac{1}{2}}^{*}}{\Delta t}=-\frac{\left(q_{c}\right)_{i, k}^{n+\frac{1}{2}}-\left(q_{c}\right)_{i, k+1}^{n+\frac{1}{2}}}{h_{i, k+\frac{1}{2}}^{n}}
\end{aligned}
$$

representing the nonhydrostatic correction step. The variables $u^{*}$ and $w^{*}$, representing the hydrostatic provisional velocities, are calculated by including all terms in the governing equations except the nonhydrostatic correction pressure, $q_{c}$. In Equations (10), the superscripts enclosed in parenthesis represent 


$$
\begin{aligned}
& (\cdot)_{i^{\prime}, k^{\prime}}^{(n)_{\mathrm{Ex}}}=\quad \frac{1}{2}(3+b)(\cdot)_{i^{\prime}, k^{\prime}}^{n}-\frac{1}{2}(1+2 b)(\cdot)_{i^{\prime}, k^{\prime}}^{n-1}+\frac{b}{2}(\cdot)_{i^{\prime}, k^{\prime}}^{n-2} \\
& (\cdot)_{i^{\prime}, k^{\prime}}^{(n+1)} \operatorname{Im}=\frac{1}{2}(1+c)(\cdot)_{i^{\prime}, k^{\prime}}^{n+1}+\frac{1}{2}(1-2 c)(\cdot)_{i^{\prime}, k^{\prime}}^{n}+\quad \frac{c}{2}(\cdot)_{i^{\prime}, k^{\prime}}^{n-1} \\
& (\cdot)_{i^{\prime}, k^{\prime}}^{\left(n+\theta^{*}\right)}=\quad \theta(\cdot)_{i^{\prime}, k^{\prime}}^{*}+(1-\theta)(\cdot)_{i^{\prime}, k^{\prime}}^{n}
\end{aligned}
$$

where superscript $n$ represents the time step in the multistep scheme and subscripts $i^{\prime}$ and $k^{\prime}$ represent dummy indices which can become any location on the staggered grid. Here, the terms discretized with $(\cdot)_{i^{\prime}, k^{\prime}}^{(n)_{\mathrm{Ex}}}$ and $(\cdot)_{i^{\prime}, k^{\prime}}^{(n+1)_{\operatorname{Im}}}$ in Equations (12) and (13), respectively, represent the explicit and implicit time stepping procedures described in Durran and Blossey (2012). The explicit (12) and implicit (13) terms in the momentum equations are weighted using parameters $b$ and $c$, respectively. For the explicit portion of the method, $b=0$ corresponds to the second-order Adams-Bashforth (AB2) method, $b=5 / 6$ is the AB3 method and $b=1 / 2$ is the AX2* method (Durran and Blossey, 2012). On the other hand, for the implicit portion of the method, $c=0$ corresponds to the trapezoidal or Crank-Nicoloson (CN) method (Crank and Nicolson, 1947), $c=1 / 2$ is the AM2* method and $c=3 / 2$ is the AI2* method (Durran and Blossey, 2012). The discretization of $(\cdot)_{i^{\prime}, k^{\prime}}^{\left(n+\theta^{*}\right)}$ given in Equation (14) represents the popular semi-implicit time stepping procedure used in Casulli and Cattani (1994). Here, $\theta$ represents the implicitness parameter, where $\theta=0$ is the forward Euler method, $\theta=1 / 2$ is the $\mathrm{CN}$ method, and $\theta=1$ is the backward Euler method. Finally, the split- 
ting of the governing equations into Equations (10) and (11) represents the nonhydrostatic projection method of Armfield and Street (2000); Armfield and Street (2003) where the nonhydrostatic pressure variables are defined at the half time-steps, $n \pm \frac{1}{2}$. For the nonhydrostatic portion of the method, the parameter $\alpha=0$ or 1 designates the use of the pressure projection (1st-order accurate) or pressure correction (2nd-order accurate) method, respectively (Armfield and Street, 2002; Kang and Fringer, 2005; Vitousek and Fringer, 2013).

The advection, diffusion, Coriolis, and surface pressure ${ }^{2}$ are discretized with the explicit method given in Equation (12). In Equations (10), $F_{i+\frac{1}{2}, k}^{n}$ and $G_{i, k+\frac{1}{2}}^{n}$ represent the terms discretized using the generalized, explicit AB method (12) and are given by

$$
\begin{aligned}
F_{i+\frac{1}{2}, k}^{n}= & -C_{H}\left(u_{i+\frac{1}{2}, k}^{n}\right)+D_{H}\left(u_{i+\frac{1}{2}, k}^{n}\right)+f v_{i+\frac{1}{2}, k}^{n}-\tilde{f} w_{i+\frac{1}{2}, k} \\
& -\frac{1}{\rho_{0}} \frac{\left(p_{s}\right)_{i+1}^{n}-\left(p_{s}\right)_{i}^{n}}{\Delta x}-\frac{\left(M^{(b c)}\right)_{i+1, k}^{n}-\left(M^{(b c)}\right)_{i, k}^{n}}{\Delta x}, \\
G_{i, k+\frac{1}{2}}^{n}= & -C_{H}\left(w_{i, k+\frac{1}{2}}^{n}\right)+D_{H}\left(w_{i, k+\frac{1}{2}}^{n}\right)+\tilde{f} u_{i, k+\frac{1}{2}}^{n},
\end{aligned}
$$

\footnotetext{
${ }^{2}$ Although the surface pressure is, technically, part of the barotropic pressure or barotropic Montgomery potential, explicit treatment of this term is common and sufficient because it does not lead to problem stiffness. In contrast, the $g \eta$ term in the barotropic Montgomery potential leads to stiffness associated with fast free-surface gravity waves and thus requires implicit discretization.
} 
where $M^{(b c)}$ is the baroclinic Montgomery potential which is given by

$$
\begin{aligned}
& M_{1}^{(b c)}=0 \\
& M_{k+1}^{(b c)}=M_{k}^{(b c)}+\frac{\left(\rho_{k+1}-\rho_{k}\right) g z_{k}}{\rho_{0}} .
\end{aligned}
$$

Equation (17) is a downward recursive relationship that allows the baroclinic Montgomery potential to be calculated from the layers above. In Equation (17), the interface locations are calculated from an upward recursive relationship given by

$$
\begin{aligned}
z_{N_{\text {layers }}} & =-H, \\
z_{k-1} & =z_{k}+h_{k},
\end{aligned}
$$

where $H$ is the depth to the seabed. $C_{H}()$ and $D_{H}()$ represent the horizontal advection and diffusion operators, respectively. Horizontal advection can be discretized with several different schemes - the simplest option being the central differencing scheme given by $C_{H}\left(u_{i+\frac{1}{2}, k}^{n}\right)=u_{i+\frac{1}{2}, k}^{n} \frac{u_{i+\frac{3}{2}, k}^{n}-u_{i-\frac{1}{2}, k}^{n}}{2 \Delta x}$. For horizontal diffusion the operator is given by $D_{H}\left(u_{i+\frac{1}{2}, k}^{n}\right)=\nu_{H} \frac{u_{i+\frac{3}{2}, k}^{n}-2 u_{i+\frac{1}{2}, k}^{n}+u_{i-\frac{1}{2}, k}^{n}}{\Delta x^{2}}$, where $\nu_{H}$ is the horizontal eddy-viscosity.

The barotropic portion of the Montgomery potential is discretized using the generalized, semi-implicit Adams-Moulton (AM) method (13) of Durran and Blossey (2012). This method is reminiscent of the $\theta$-method of Casulli (1990) and is intended to eliminate the time step restriction associated with 
fast free-surface gravity waves. The generalized, explicit/implicit method used here is preferable to popular methods such as Leapfrog-Trapezoidal ${ }^{3}$ because it does not require filtering. It is also preferable to AB2(3)- $\theta$ method $^{4}$ which is unstable for stiff problems in the inviscid limit when $\theta=0.5$ (Durran, 1991). By using the generalized, explicit/implicit method, the current model can run stably without horizontal or vertical viscosity.

Isopycnal or layered models must use implicit discretization for vertical diffusion of momentum when the isopycnal layers are allowed to become arbitrarily thin (Hallberg, 2000). Following Casulli (1997), the viscous boundary condition at the free surface is specified by applying a wind stress given as

$$
\begin{aligned}
& \left(\tau^{x}\right)_{i+\frac{1}{2}, 1 / 2}^{*}=\gamma_{w}\left(\left(u_{w}\right)_{i+\frac{1}{2}}^{n+1}-u_{i+\frac{1}{2}, 1}^{*}\right) \\
& \left(\tau^{x}\right)_{i+\frac{1}{2}, 1 / 2}^{n}=\gamma_{w}\left(\left(u_{w}\right)_{i+\frac{1}{2}}^{n}-u_{i+\frac{1}{2}, 1}^{n}\right)
\end{aligned}
$$

where $\gamma_{w}$ is the wind stress coefficient and $u_{w}$ is the prescribed wind speed. The boundary condition at the seabed is specified by applying the quadratic drag law

$$
\begin{aligned}
& \left(\tau^{x}\right)_{i+\frac{1}{2}, N_{\text {layers }}+1 / 2}^{*}=C_{d}\left|u_{i+\frac{1}{2}, N_{\text {layers }}}^{n}\right| u_{i+\frac{1}{2}, N_{\text {layers }}}^{*}, \\
& \left(\tau^{x}\right)_{i+\frac{1}{2}, N_{\text {layers }}+1 / 2}^{n}=C_{d}\left|u_{i+\frac{1}{2}, N_{\text {layers }}}^{n}\right| u_{i+\frac{1}{2}, N_{\text {layers }}}^{n},
\end{aligned}
$$

\footnotetext{
${ }^{3}$ Leapfrog is used for the explicit terms and the trapezoidal method is used for the implicit terms.

${ }^{4} \mathrm{AB} 2$ or AB3 is used for the explicit terms and the $\theta$-method is used for the implicit terms.
} 
where $C_{d}$ is the drag coefficient. In Equation (21a), the term representing the velocity magnitude is taken at time step $n$ as is common for implicit discretization of the drag term. This time-lagging approach removes the need to solve a nonlinear system of equations for the horizontal velocity. For the vertical momentum equation, there is no stress applied in the vertical direction at the surface, $\tau_{i,-\frac{1}{2}}^{z}=0$, and at the bottom, $\tau_{i, N_{\text {layers }}+\frac{1}{2}}^{z}=0$. The shear stress between layers in the horizontal momentum equation is given by

$$
\begin{aligned}
& \left(\tau^{x}\right)_{i+\frac{1}{2}, k+\frac{1}{2}}^{*}=\left(\nu_{v}\right)_{k+\frac{1}{2}} \frac{u_{i+\frac{1}{2}, k}^{*}-u_{i+\frac{1}{2}, k+1}^{*}}{h_{i+\frac{1}{2}, k+\frac{1}{2}}^{n}}, \\
& \left(\tau^{x}\right)_{i+\frac{1}{2}, k+\frac{1}{2}}^{n}=\left(\nu_{v}\right)_{k+\frac{1}{2}} \frac{u_{i+\frac{1}{2}, k}^{n}-u_{i+\frac{1}{2}, k+1}^{n}}{h_{i+\frac{1}{2}, k+\frac{1}{2}}^{n}} .
\end{aligned}
$$

In the vertical momentum equation, the shear stress between layers is given by

$$
\begin{aligned}
\left(\tau^{z}\right)_{i, k}^{*} & =\left(\nu_{v}\right)_{k} \frac{w_{i, k-\frac{1}{2}}^{*}-w_{i, k+\frac{1}{2}}^{*}}{h_{i, k}^{n}} \\
\left(\tau^{z}\right)_{i, k}^{n} & =\left(\nu_{v}\right)_{k} \frac{w_{i, k-\frac{1}{2}}^{n}-w_{i, k+\frac{1}{2}}^{n}}{h_{i, k}^{n}}
\end{aligned}
$$

In Equations (22a) and (23a), the layer height $h$ is taken at time step $n$ to eliminate the need to solve a fully nonlinear system.

In order to demonstrate the numerical procedure used with implicit vertical diffusion, it is helpful to use matrix notation. After substituting Equa- 


$$
\begin{aligned}
\mathbf{T}_{i+\frac{1}{2}}^{n}=\mathbf{u}_{i+\frac{1}{2}}^{n} & -\frac{1}{2}(1-2 c) g \frac{\Delta t}{\Delta x}\left(\eta_{i+1}^{n}-\eta_{i}^{n}\right) \mathbf{e}-\frac{c}{2} g \frac{\Delta t}{\Delta x}\left(\eta_{i+1}^{n-1}-\eta_{i}^{n-1}\right) \mathbf{e} \\
& +\Delta t \mathbf{F}_{i+\frac{1}{2}}^{(n)_{\operatorname{Ex}}}-\alpha \frac{\Delta t}{\Delta x}\left(\mathbf{q}_{i+1}^{n-\frac{1}{2}}-\mathbf{q}_{i}^{n-\frac{1}{2}}\right)+\Delta t(1-\theta)\left(\mathbf{D}_{\mathbf{A}}\right)_{i+\frac{1}{2}} \mathbf{u}_{i+\frac{1}{2}}^{n}
\end{aligned}
$$

$$
\mathbf{S}_{i}^{n}=\mathbf{w}_{i}^{n}+\Delta t \mathbf{G}_{i}^{(n)_{\mathrm{Ex}}}-\alpha \Delta t \mathbf{D}_{z}\left(\mathbf{q}_{i}^{n-\frac{1}{2}}\right)+\Delta t(1-\theta)\left(\mathbf{D}_{\mathbf{B}}\right)_{i} \mathbf{w}_{i}^{n}
$$

386

tions (22) and (23) and the boundary conditions for the shear stress, $\tau$, the hydrostatic portion of the discrete momentum equations (10) can be written in matrix form as

$$
\begin{aligned}
\mathbf{A}_{i+\frac{1}{2}} \mathbf{u}_{i+\frac{1}{2}}^{*} & =\mathbf{T}_{i+\frac{1}{2}}^{n}-\frac{1}{2}(1+c) g \frac{\Delta t}{\Delta x}\left(\eta_{i+1}^{n+1}-\eta_{i}^{n+1}\right) \mathbf{e} \\
\mathbf{B}_{i} \mathbf{w}_{i}^{*} & =\mathbf{S}_{i}^{n}
\end{aligned}
$$

where $\mathbf{u}_{i+\frac{1}{2}}^{*}=\left[u_{i+\frac{1}{2}, 1}^{*}, u_{i+\frac{1}{2}, 2}^{*}, \ldots, u_{i+\frac{1}{2}, k}^{*}, \ldots, u_{i+\frac{1}{2}, N_{\text {layers }}}^{*}\right]^{T}$ and $\mathbf{w}_{i}^{*}=\left[w_{i, \frac{1}{2}}^{*}, w_{i, \frac{3}{2}}^{*}, \ldots, w_{i, k+\frac{1}{2}}^{*}, \ldots, w_{i, N_{\text {layers }}+\frac{1}{2}}^{*}\right]^{T}$ are the hydrostatic predictor $\left(^{*}\right)$ velocities throughout a column of grid points. The equations for $\mathbf{u}_{i+\frac{1}{2}}^{n+1}$ and $\mathbf{w}_{i}^{n+1}$ based on Equations (11) are detailed in Section 3.3. Vectors $\mathbf{T}_{i+\frac{1}{2}}^{n}$ and $\mathbf{S}_{i}^{n}$ contain all explicit terms of the momentum equations (10) which include the $\mathrm{AB}$ terms (at time levels $n, n-1$, and $n-2$ ) as well as the explicit parts of the time derivative, semi-implicit free-surface method (13), $\theta$-method (14), and nonhydrostatic pressure, viz.

where $\mathbf{u}_{i+\frac{1}{2}}^{n}, \mathbf{F}_{i+\frac{1}{2}}^{n}$ and $\mathbf{w}_{i}^{n}, \mathbf{G}_{i}^{n}$ are defined similar to $\mathbf{u}_{i+\frac{1}{2}}^{*}$ and $\mathbf{w}_{i}^{*}$ (i.e. they 
represent the variables of a column of grid points) and $\mathbf{e}=[1,1, \ldots, 1]^{T} \in$ $\Re^{N_{\text {layers }} \times 1}$ and $\mathbf{D}_{z}\left(\mathbf{q}_{i}^{n-\frac{1}{2}}\right)=\left(q_{i, k}^{n-\frac{1}{2}}-q_{i, k+1}^{n-\frac{1}{2}}\right) / h_{i, k}^{n} \quad \forall k$ represents the vertical first derivative. The matrices $\mathbf{A}$ and $\mathbf{B}$, given by

$$
\begin{aligned}
& \mathbf{A}_{i+\frac{1}{2}}=\mathbf{I}-\Delta t \theta\left(\mathbf{D}_{\mathbf{A}}\right)_{i+\frac{1}{2}}, \\
& \mathbf{B}_{i}=\mathbf{I}-\Delta t \theta\left(\mathbf{D}_{\mathbf{B}}\right)_{i},
\end{aligned}
$$

with $\mathbf{D}_{\mathbf{A}}$ and $\mathbf{D}_{\mathbf{B}}$ being tridiagonal matrices of the form

$$
\begin{aligned}
& \left(\mathbf{D}_{\mathbf{A}}\right)_{i+\frac{1}{2}}=\operatorname{tridiag}\left[\frac{\left(\nu_{v}\right)_{k-\frac{1}{2}}}{h_{i+\frac{1}{2}, k}^{n} h_{i+\frac{1}{2}, k-\frac{1}{2}}^{n}},-\left(\frac{\left(\nu_{v}\right)_{k-\frac{1}{2}}}{h_{i+\frac{1}{2}, k}^{n} h_{i+\frac{1}{2}, k-\frac{1}{2}}^{n}}+\frac{\left(\nu_{v}\right)_{k+\frac{1}{2}}}{h_{i+\frac{1}{2}, k}^{n} h_{i+\frac{1}{2}, k+\frac{1}{2}}^{n}}\right), \frac{\left(\nu_{v}\right)_{k+\frac{1}{2}}}{h_{i+\frac{1}{2}, k}^{n} h_{i+\frac{1}{2}, k+\frac{1}{2}}^{n}}\right], \\
& \left(\mathbf{D}_{\mathbf{B}}\right)_{i}=\operatorname{tridiag}\left[\frac{\left(\nu_{v}\right)_{k}}{h_{i, k+\frac{1}{2}}^{n} h_{i, k}^{n}},-\left(\frac{\left(\nu_{v}\right)_{k}}{h_{i, k+\frac{1}{2}}^{n} h_{i, k}^{n}}+\frac{\left(\nu_{v}\right)_{k+1}}{h_{i, k+\frac{1}{2}}^{n} h_{i, k+1}^{n}}\right), \frac{\left(\nu_{v}\right)_{k+1}}{h_{i, k+\frac{1}{2}}^{n} h_{i, k+1}^{n}}\right]
\end{aligned}
$$

represent the implicit discretization of vertical diffusion of momentum. Note that the first and last diagonal elements of the tridiagonal matrix (27a) must be modified to account for the proper boundary conditions in Equations (20) and (21), respectively. When a variable is needed that is not located at the position on the grid where it is defined (such as $h_{i \pm \frac{1}{2}, k}, h_{i, k \pm \frac{1}{2}}$, or $h_{i \pm \frac{1}{2}, k \pm \frac{1}{2}}$ ), then it is approximated with linear interpolation. Finally, the momentum 
397

equations (24) can be written as

$$
\begin{aligned}
\mathbf{u}_{i+\frac{1}{2}}^{*} & =\tilde{\mathbf{T}}_{i+\frac{1}{2}}^{n}-\frac{1}{2}(1+c) g \frac{\Delta t}{\Delta x}\left(\eta_{i+1}^{n+1}-\eta_{i}^{n+1}\right) \tilde{\mathbf{e}}_{i+\frac{1}{2}} \\
\mathbf{w}_{i}^{*} & =\tilde{\mathbf{S}}_{i}^{n}
\end{aligned}
$$

398 where

$$
\begin{aligned}
\tilde{\mathbf{T}}_{i+\frac{1}{2}}^{n} & =\mathbf{A}_{i+\frac{1}{2}}^{-1} \mathbf{T}_{i+\frac{1}{2}}^{n} \\
\tilde{\mathbf{e}}_{i+\frac{1}{2}} & =\mathbf{A}_{i+\frac{1}{2}}^{-1} \mathbf{e} \\
\tilde{\mathbf{S}}_{i}^{n} & =\mathbf{B}_{i}^{-1} \mathbf{S}_{i}^{n}
\end{aligned}
$$

399

represent the solutions following the tridiagonal inversion for each column of grid points in the horizontal.

\subsection{Discretized free-surface and layer-height equations}

In isopycnal models the layer height continuity equation (7) is used to evolve both the free surface and the internal interface (Adcroft and Hallberg, 2006). In the present model, we differentiate the free surface from internal interfaces. Accordingly, we derive separate but consistent model equations for the evolution of the internal layers heights $h$ and free surface, $\eta$, both of which use the generalized, semi-implicit AM method of Durran and Blossey (2012).

The layer height continuity equation (7) is discretized with the generalized 
410

semi-implicit AM method and is given by

$$
\frac{h_{i, k}^{n+1}-h_{i, k}^{n}}{\Delta t}=-\frac{1}{\Delta x}\left(h_{i+\frac{1}{2}, k}^{n} u_{i+\frac{1}{2}, k}^{(n+1)_{\operatorname{Im}^{*}}}-h_{i-\frac{1}{2}, k}^{n} u_{i-\frac{1}{2}, k}^{(n+1)_{\operatorname{Im}^{*}}}\right),
$$

where $u_{i \pm \frac{1}{2}}^{(n+1)_{\mathrm{Im}^{*}}}=\frac{1}{2}(1+c) u_{i \pm \frac{1}{2}}^{*}+\frac{1}{2}(1-2 c) u_{i \pm \frac{1}{2}}^{n}+\frac{c}{2} u_{i \pm \frac{1}{2}}^{n-1}$ following Equation (13) and using the hydrostatic predictor velocity $u^{*}$ instead of the final velocity $u^{n+1}$. Using a first-order upwind approach to calculate $h_{i \pm \frac{1}{2}, k}^{n}$ and assuming a positive flow direction, Equation (30) (following the approach of Stelling and Duinmeijer (2003)) can be written as

$$
h_{i, k}^{n+1}=\left(1-\frac{u_{i+\frac{1}{2}, k}^{(n+1) \operatorname{Im}^{*}} \Delta t}{\Delta x}\right) h_{i, k}^{n}+\frac{u_{i-\frac{1}{2}, k}^{(n+1) \operatorname{Im}^{*}} \Delta t}{\Delta x} h_{i-1, k}^{n},
$$

with $u_{i \pm \frac{1}{2}, k}^{(n+1)_{\operatorname{Im} *}} \geq 0$. A similar form can be obtained for $u_{i \pm \frac{1}{2}, k}^{(n+1)_{\operatorname{Im}}} \leq 0$. As seen in Equation (31), as long as the advective Courant number $U_{i+\frac{1}{2}, k}^{(n+1) \operatorname{Im}^{*}}=$ $\frac{u_{i+\frac{1}{2}, k}^{(n+1)} \operatorname{Im}^{*} \Delta t}{\Delta x} \leq 1$ then positive water depths are obtained without any special treatment for wetting and drying (Stelling and Duinmeijer, 2003). We can apply MPDATA corrections (outlined in Appendix C) to the first-order upwinding approach to ensure that the method is second-order accurate in time and space and the layer heights remain positive.

The equation governing the evolution of the free surface is derived by summing Equation (30) over the layer index $k$. The resulting free-surface 
equation is given by

$$
-\left(\alpha_{1}\right)_{i-\frac{1}{2}}^{n} \eta_{i-1}^{n+1}+\left[1+\left(\alpha_{1}\right)_{i-\frac{1}{2}}^{n}+\left(\alpha_{1}\right)_{i+\frac{1}{2}}^{n}\right] \eta_{i}^{n+1}-\left(\alpha_{1}\right)_{i+\frac{1}{2}}^{n} \eta_{i+1}^{n+1}=R_{i}^{n}
$$

which is a tridiagonal equation in one horizontal dimension or a block tridiagonal in 3-D. The coefficients $\left(\alpha_{1}\right)_{i \pm \frac{1}{2}}^{n}=g\left[\frac{1}{2}(1+c) \frac{\Delta t}{\Delta x}\right]^{2} \mathbf{e}^{T}\left(\mathbf{h}_{i \pm \frac{1}{2}}^{n} \circ \tilde{\mathbf{e}}_{i \pm \frac{1}{2}}\right)$ (where $\circ$ is the element-wise product) and the right-hand side $R_{i}^{n}$ are derived in Appendix B.

Because both the free-surface equation (32) and the sum of the layer heights given by Equation (30) provide an estimate of the free-surface height, $\eta$, these estimates can become inconsistent over the course of a simulation. Models that use a mode-split time-stepping procedure are susceptible to this inconsistency, as discussed at length by Hallberg and Adcroft (2009). In the present model, consistency between the free-surface equation (32) and the layer-height equation (30) is ensured as long as the same flux face heights, $h_{i \pm \frac{1}{2}, k}^{n}$, are used in each equation ${ }^{5}$. However, when using upwinding or MPDATA for Equation (30) or solving the free-surface equation (32) (in 2-D) using a non-exact (iterative) method such as conjugate gradients, this is generally not the case. Notice how according to Equation (30) and (31) we seek to upwind the flux face heights, $h_{i \pm \frac{1}{2}, k}^{n}$, using the velocity $u_{i \pm \frac{1}{2}, k}^{(n+1)_{\operatorname{Im} *}}$. This velocity and thus $h_{i \pm \frac{1}{2}, k}^{n}$ are unknown ${ }^{6}$ when the free-surface equation (32) is

\footnotetext{
${ }^{5}$ naturally, since the free-surface equation is derived from the layer-height equation.

${ }^{6}$ The initial flux face heights, $h_{i \pm \frac{1}{2}, k}^{n}$, and thus $\alpha$ and $R$ in the free-surface equation (32)
} 
solved because the velocity $u^{(n+1)_{\mathrm{Im}^{*}}}$ depends on $u^{*}$ which depends on $\eta^{n+1}$ according to Equation (28a). There are a few methods that can be used to remove the inconsistency between the free-surface equation (32) and the layer-height equation (30). One option is to use an iterative procedure between the free-surface equation (32) and the layer-height equation (30). The values of $h_{i \pm \frac{1}{2}, k}^{n}$ (based on upwinding of the velocity $u_{i \pm \frac{1}{2}, k}^{(n+1) \mathrm{Im}^{*}}$ ) can be reused in the free-surface equation and the entire procedure can be iterated until convergence is reached. This procedure is outlined in detail in Section 3.4. In general, we have found that this iterative procedure converges quite rapidly in $\mathcal{O}(1)$ iterations. Other non-iterative options to remove this inconsistency include: [1] upwind the flux face heights based on the $u^{n}$ velocity; [2] directly set the free surface $\eta$ to $\sum h_{k}-H_{i}$, where $H_{i}$ is the still water depth at location $i$; or [3] apply a uniform expansion/contraction of the isopycnal layers to fit the total depth according to $h_{k}=\left(\frac{\eta+H}{\sum h_{k}}\right) h_{k}$ (Bleck and Smith, 1990; Hofmeister et al., 2010). Method [3] is generally the preferred method used in existing isopycnal models.

\subsection{Nonhydrostatic pressure}

Equations (28) provide the hydrostatic predictor portion of the numerical solution. To obtain the nonhydrostatic solution, the hydrostatic predictor velocities are corrected according to Equations (11) in order to satisfy

are approximated using the upwinded layer heights based on the $u_{i \pm \frac{1}{2}, k}^{n}$ velocity instead of the $u_{i \pm \frac{1}{2}, k}^{(n+1)_{\mathrm{Im}^{*}}}$ velocity which is calculated later. 
the divergence-free constraint. The nonhydrostatic correction pressure $q_{c}$ is determined from the solution of a Poisson equation which is derived by substituting the nonhydrostatic correction velocities, $u_{i \pm \frac{1}{2}, k}^{n+1}$ and $w_{i, k \pm \frac{1}{2}}^{n+1}$, from Equations (11) into the discrete divergence-free condition which, as derived in Appendix D, is subject to a mild-slope approximation and is given by

$$
\frac{1}{\Delta x}\left(h_{i+\frac{1}{2}, k}^{n+1} u_{i+\frac{1}{2}, k}^{n+1}-h_{i-\frac{1}{2}, k}^{n+1} u_{i-\frac{1}{2}, k}^{n+1}\right)+w_{i, k-\frac{1}{2}}^{n+1}-w_{i, k+\frac{1}{2}}^{n+1}=0 .
$$

Substituting (11) into (33) results in a symmetric linear system of equations for the nonhydrostatic pressure $q_{c}$ that is given by

$$
\begin{aligned}
& \lambda_{i, k-\frac{1}{2}}^{n+1}\left(q_{c}\right)_{i, k-1}^{n+\frac{1}{2}}+\frac{1}{\lambda_{i-\frac{1}{2}, k}^{n+1}}\left(q_{c}\right)_{i-1, k}^{n+\frac{1}{2}} \\
& -\left(\lambda_{i, k-\frac{1}{2}}^{n+1}+\lambda_{i, k+\frac{1}{2}}^{n+1}+\frac{1}{\lambda_{i-\frac{1}{2}, k}^{n+1}}+\frac{1}{\lambda_{i+\frac{1}{2}, k}^{n+1}}\right)\left(q_{c}\right)_{i, k}^{n+\frac{1}{2}} \\
& +\frac{1}{\lambda_{i+\frac{1}{2}, k}^{n+1}}\left(q_{c}\right)_{i+1, k}^{n+\frac{1}{2}}+\lambda_{i, k+\frac{1}{2}}^{n+1}\left(q_{c}\right)_{i, k+1}^{n+\frac{1}{2}} \\
& =\frac{\Delta x}{\Delta t}\left[\frac{1}{\Delta x}\left(h_{i+\frac{1}{2}, k}^{n+1} u_{i+\frac{1}{2}, k}^{*}-h_{i-\frac{1}{2}, k}^{n+1} u_{i-\frac{1}{2}, k}^{*}\right)+w_{i, k-\frac{1}{2}}^{*}-w_{i, k+\frac{1}{2}}^{*}\right]
\end{aligned}
$$

In Equation (34), $\lambda=\Delta x / h$ is the grid leptic ratio or lepticity (Scotti and Mitran, 2008) of the isopycnal layer. This parameter is a measure of the ratio of numerical to physical dispersion (Vitousek and Fringer, 2011) and sets the anisotropy of the elliptic equation (34). Derivation of the full elliptic equation in isopycnal coordinates (without the mild-slope approximation) 
is presented in Appendix E. Equation (34) is quite similar to the elliptic equation derived from $z$-coordinates. The only significant difference is that, here, the coefficients are based on the time-variable grid aspect-ratio $\lambda=$ $\Delta x / h$ where as in $z$-coordinates the grid aspect ratio $(\Delta x / \Delta z)$ is fixed in time. Since the condition number of the linear system is related to the grid aspect ratio (Marshall et al., 1997b; Kramer et al., 2010; Fiebig-Wittmaack et al., 2011), time variability of the coefficients of Equation (34) results in time variability of the condition number of the linear system. However, we find that popular elliptic solvers, such as preconditioned conjugate gradients or multigrid with semi-coarsening and line-relaxation ${ }^{7}$ (Briggs et al., 2000), are suitable to solving Equation (34) efficiently.

As discussed previously, Adcroft and Hallberg (2006) conclude that the nonhydrostatic equations and the LVD approach (using a prognostic equation for $h^{n+1}$ as in Equation (30)) are mutually exclusive. The present study is not limited by this issue. As mentioned in Section 1.3, the two forms of the continuity equation, the layer-height equation (30) and the divergencefree equation (33), assume different roles in the numerical method. In fact, the numerical method in current approach is remarkably similar to nonhydrostatic methods with a free surface in z-coordinates. Here, the hydrostatic portion of the method advances the free surface and layer heights (or the free surface alone when using $z$-coordinates). Next, the nonhydrostatic method is

\footnotetext{
${ }^{7}$ a method developed for anisotropic problems
} 
used to correct the velocities while holding the layers fixed. As a result, the final free surface and layer heights are not consistent with the layer-height continuity equation in terms of the final velocities (at time $n+1$ ). A "fully" nonhydrostatic method would require iteration of the elliptic and layer-height equations. However, holding the free surface fixed during the nonhydrostatic portion of the pressure correction method in $z$-coordinates does not impact the order of accuracy (Armfield and Street, 2000; Kang and Fringer, 2005; Vitousek and Fringer, 2013). The convergence analysis presented in Section 4.2 demonstrates that this is also the case when fixing the layer heights during the nonhydrostatic portion of the method in isopycnal coordinates.

\subsection{Solution procedure}

The solution procedure for one time step of the present model is given by:

1. Calculate the explicit portions of the horizontal and vertical momentum equations, $\mathbf{T}^{n}$ and $\mathbf{S}^{n}$, using Equations (25).

2. Solve the implicit vertical diffusion equations (29) to compute $\tilde{\mathbf{T}}_{i+\frac{1}{2}}^{n}$, $\tilde{\mathbf{e}}_{i+\frac{1}{2}}$, and $\tilde{\mathbf{S}}_{i}^{n}$ for each column of grid points in the horizontal where the tridiagonal matrices $\mathbf{A}_{i+\frac{1}{2}}$ and $\mathbf{B}_{i}$, given Equations (26a) and (26b), respectively, represent implicit discretization of vertical diffusion.

3. Solve the implicit free-surface equation (32) to obtain the free surface height at the next time step, $\eta^{n+1}$. 
4. Compute the hydrostatic predictor velocities $\mathbf{u}_{i+\frac{1}{2}}^{*}$ and $\mathbf{w}_{i}^{*}$ using Equations (28).

5. Compute the layer heights, $h^{n+1}$ using Equation (30) and (optionally) the MPDATA procedure described in Appendix C.

6. If an iterative procedure is used to obtain consistency between $\eta^{n+1}$ and $h^{n+1}$, return to step 3 , where the $\alpha_{1}$ coefficients in the free-surface equation (32) are updated based on upwinding the velocities $\left(u^{(n+1) \operatorname{Im}^{*}}=\mathrm{f}\left(u^{*}, u^{n}, u^{n-1}\right)\right)$ obtained in step 4. If the iterate values for $h^{n+1}$ have converged to some tolerance, continue to step 7. If a non-iterative procedure is used, set $h_{k}^{n+1}=\left(\frac{\eta^{n+1}+H}{\sum h_{k}^{n+1}}\right) h_{k}^{n+1}$ and continue.

7. Update the interface locations, $z$ (see Figure 1), and the baroclinic Montgomery potentials, $M^{(\mathrm{bc})}$, using Equations (19) and (17), respectively.

8. For the nonhydrostatic method, solve the Poisson equation (34) for the nonhydrostatic correction pressure $\left(q_{c}^{n+\frac{1}{2}}\right)$, correct the horizontal predictor (hydrostatic) velocities using the nonhydrostatic pressure (11a), and set $q^{n+\frac{1}{2}}=\alpha q_{c}^{n+\frac{1}{2}}+q^{n-\frac{1}{2}}$. To run the model in hydrostatic mode, this step is ignored; $q_{c}=0$ and $u^{n+1}=u^{*}$.

9. Finally, compute the vertical velocity by integrating the continuity equation (33) from the bottom grid cell (where $w_{i, N_{\text {layers }}+\frac{1}{2}}=0$ ) upward. 


\section{Stability and convergence}

\subsection{Stability}

The current model is susceptible to both physical and numerical instabilities. The physical instability is due to the formation of Kelvin-Helmholtz $(\mathrm{KH})$ billows that may occur when the Richardson number $(R i)$ is less than 1/4 (Miles, 1961; Howard, 1961). Numerical instability, on the other hand, is related to the Courant numbers for the time stepping of terms such as the baroclinic pressure gradient, advection of momentum, horizontal viscosity or updating the layer heights.

Although nonhydrostatic models using $z$ - and $\sigma$-coordinates are capable of resolving $\mathrm{KH}$ billows, layered and isopycnal coordinate formulations are not. The formation of $\mathrm{KH}$ instabilities renders layered models ill-posed and unstable (Grue et al., 1997; Jo and Choi, 2002; Castro-Díaz et al., 2011; Mandli, 2013). Chumakova et al. (2009) showed that the condition for instability of the inviscid, multilayer shallow water (i.e. hydrostatic) equations is $R i<1 / 4$. Barad and Fringer (2010) found that KH billows develop on internal solitary waves when $R i<0.1$. The appearance of $\mathrm{KH}$ instabilities in layered models can be suppressed by adding viscosity (Castro-Díaz et al., 2011), filtering (Jo and Choi, 2008) or adopting a different numerical formulation (Choi et al., 2009). The current model does not take active measures to remove this physical instability. Instead, the model is typically applied in regimes where $R i>1 / 4$ and $\mathrm{KH}$ billows do not exist. However, in general, we do not attempt to proactively avoid dynamical situations where KH bil- 
lows develop. As is typically the case in hydrostatic isopycnal models, we have found that the small amount of damping due to viscosity, drag, and the numerical schemes is usually sufficient to suppress instability due to the KH mechanism.

The numerical stability of the time-stepping method used in the current model is investigated in Durran and Blossey (2012). For a 1-D stiff, linear model problem, Durran and Blossey (2012) determined a maximum Courant number of 0.76 and 0.72 for the $\mathrm{AM} 2{ }^{*}-\mathrm{AX} 2 *$ and $\mathrm{AI} 2{ }^{*}-\mathrm{AB} 3$ methods, respectively. We generally find that the 2-D model is stable for nonlinear simulations when the internal Courant number, $C_{i}=\frac{c_{i} \Delta t}{\Delta x}<0.5$ where $c_{i}$ is the maximum (mode-1) internal wave speed. We also find that the model is stable when the horizontal diffusion Courant number $C_{\nu_{H}}=\frac{2 \nu_{H} \Delta t}{\Delta x^{2}}<0.25$ when using AB3. Additionally, we require that the horizontal advective Courant number $C_{u}=\frac{u \Delta t}{\Delta x} \leq 1$ to ensure positive layer heights according to Equation (31). There is no time step restriction associated with vertical advection since such terms do not appear in the governing equations (6). In 3 -D, the maximum Courant numbers may be reduced even further. In general, there is no restriction on the free-surface Courant number since the time stepping method for the surface gravity-wave terms is A-stable (Durran and Blossey, 2012). 


\subsection{Convergence}

As mentioned in Section 3.1, the numerical method is second-order accurate in time, space, and the number of layers. To demonstrate this convergence rate, the model is run with an idealized, inviscid two-layer internal seiche in an enclosed domain as the temporal, horizontal, and vertical model resolution is increased. In the following convergence tests, the measure of convergence of the solution is obtained by

$$
\text { Error }=\operatorname{solution}(\Delta t, \Delta x, \text { or } \Delta h)-\operatorname{solution}(\Delta t / 2, \Delta x / 2, \text { or } \Delta h / 2),
$$

where "solution" means the numerical solution of variables of interest such as $\eta, \xi, u, w$ and $q$ at the final time $t_{\max }$ with a time step of $\Delta t$, a grid spacing of $\Delta x$, or a layer spacing of $\Delta h$, respectively.

For the convergence study and the following numerical experiments, we use the AM2*-AB3 numerical method, for which $c=1 / 2$ and $b=5 / 6$ (Durran and Blossey, 2012). The convergence analysis shown in Figure 2 is performed for the evolution of an internal seiche initialized with a cosine wave of the form $\xi=z_{1}+H / 2=a_{i} \cos (\pi x / L)\left(z_{1}\right.$ is the location of the internal interface, $H$ is the depth, and $L$ is the domain length) with upper- and lower-layer densities given by $\rho_{1}=1000 \mathrm{~kg} \mathrm{~m}^{-3}$ and $\rho_{2}=1001 \mathrm{~kg} \mathrm{~m}^{-3}$, respectively. The problem setup is similar to the configuration shown in Figure 3 D. We use the pressure correction method, $\alpha=1$, and an iterative procedure for the free surface with MPDATA. 


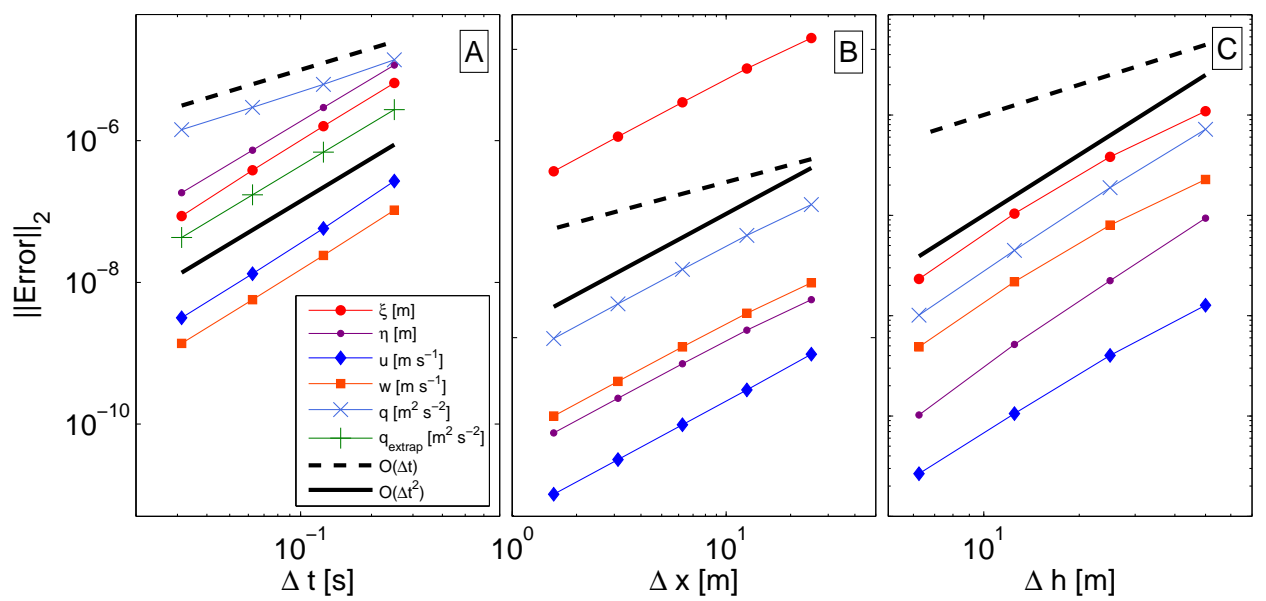

Figure 2: The temporal, horizontal and vertical resolution convergence analysis in panels $\mathrm{A}, \mathrm{B}$ and $\mathrm{C}$, respectively. The convergence analysis shows that the method converges with second-order accuracy in both time and space.

The temporal convergence experiment, shown in Figure $2 \mathrm{~A}$, is run with $a_{i}=1 \mathrm{~m}, L=1000 \mathrm{~m}$, and $H=100 \mathrm{~m}$ and with $N_{x}=128$ grid points in the horizontal and 2 layers in the vertical for various time step sizes $\Delta t=\left[\frac{1}{32}, \frac{1}{16}, \frac{1}{8}, \frac{1}{4}, \frac{1}{2}\right] \mathrm{s}$ with $t_{\max }=50 \mathrm{~s}$. As shown in Figure $2 \mathrm{~A}$, the numerical solution gives $\mathcal{O}\left(\Delta t^{2}\right)$ convergence with refinement of the time step size, $\Delta t$, as expected. In Figure $2 \mathrm{~A}$, the first-order convergence rate of the nonhydrostatic pressure, $q$, arises because the nonhydrostatic pressure is defined at time $n+\frac{1}{2}$ (Armfield and Street, 2003; Fringer et al., 2006). If this pressure is extrapolated to the final time $t_{\max }=n_{\max } \Delta t$ using $q_{\text {extrap }}=\frac{3}{2} q^{n_{\max }-\frac{1}{2}}-\frac{1}{2} q^{n_{\max }-\frac{3}{2}}+\mathcal{O}\left(\Delta t^{2}\right)$, then $\mathcal{O}\left(\Delta t^{2}\right)$ convergence is achieved as demonstrated by the slope of $q_{\text {extrap }}$ in Figure 2 A.

The horizontal grid refinement convergence experiment, shown in Figure $2 \mathrm{~B}$, is run with $a_{i}=1 \mathrm{~m}, L=100 \mathrm{~m}$ and $H=100 \mathrm{~m}$ and a small fixed time 
step of $\Delta t=0.01 \mathrm{~s}$ for 100 time steps. The model is run with 2 vertical layers and a variable number of horizontal grid points $N_{x}=[4,8,16,32,64,128]$. Because the model uses a staggered grid, variables located at cell centers such as $\eta$ are interpolated to the location $x=0$ using AB2 $\eta_{(x=0)}=\frac{3}{2} \eta_{1}-$ $\frac{1}{2} \eta_{2}+\mathcal{O}\left(\Delta x^{2}\right)$. As shown in Figure 2 B, the numerical solution gives $\mathcal{O}\left(\Delta x^{2}\right)$ convergence with refinement of the horizontal grid spacing, $\Delta x$, as expected.

The vertical layer refinement convergence experiment, shown in Figure $2 \mathrm{C}$, is run with $a_{i}=1 \mathrm{~m}, L=100 \mathrm{~m}$ and $H=100 \mathrm{~m}$ and a small fixed time step of $\Delta t=0.01 \mathrm{~s}$ for 100 time steps. The model is run with a fixed horizontal resolution of $N_{x}=512$ and variable number of layers $N_{\text {layers }}=$ $[2,4,8,16,32]$. We note that although the model may have more than two layers, the density only changes across the internal interface. The density of the upper half of the layers is $\rho_{1}=1000 \mathrm{~kg} \mathrm{~m}^{-3}$ and the density of the lower half of the layers is $\rho_{2}=1001 \mathrm{~kg} \mathrm{~m}^{-3}$. The variables of interest are interpolated to the location of the free surface $\eta$ or internal interface $\xi$ at the final time $t_{\max }$ with a vertical layer resolution of $\Delta h=H / N_{\text {layers }}$. As shown in Figure $2 \mathrm{C}$, the numerical solution gives $\mathcal{O}\left(\Delta h^{2}\right)$ convergence with refinement of the layer heights, $\Delta h$, as expected.

\section{Numerical experiments}

In this section, numerical experiments are conducted to verify the model. The test cases employed here are: (1) free-surface, (2) two-layer, and (3) continuously stratified seiches in enclosed domains; the formation of internal 
solitary waves (4) following KdV theory, (5) following Vitousek and Fringer (2011), and (6) following the laboratory experiments of Horn et al. (2001); (7) internal wave generation over a ridge in the South China Sea after Buijsman et al. (2010); (8) the formation of internal wave beams generated from oscillatory flow over a sill [see e.g. Kundu (1990)].

All of the numerical experiments use the AM2*-AB3 numerical method (for which $c=1 / 2$ and $b=5 / 6$ (Durran and Blossey, 2012)), the pressure correction method $(\alpha=1)$, the iterative procedure for the free surface and the upwind method to compute the flux-face heights unless stated otherwise. All of the numerical experiments are run with a free surface and without surface pressure, $p_{s}=0$, or Coriolis force, $f=\tilde{f}=0$.

\subsection{Free-surface seiche}

In the following sections (5.1-5.3), the (inviscid) isopycnal model is validated against linear gravity-wave theory. The dispersion relations used in the numerical experiments in Sections 5.1-5.3 are listed in Table 1.

Table 1: Dispersion relations for numerical experiments in Sections 5.1-5.3.

\begin{tabular}{llccc}
\hline Section & Sieche test case & $c$ (wave speed) & $c_{h}=\lim _{k H \rightarrow 0} c$ (shallow-water limit) & $c_{d}=\lim _{k H \rightarrow \infty} c$ (deep-water limit) \\
\hline 5.1 & Free-surface & $\sqrt{\frac{g}{k} \tanh (k H)}$ & $\sqrt{g H}$ & $\sqrt{\frac{g}{k}}$ \\
5.2 & Two-layer internal & $\sqrt{\frac{g^{\prime}}{2 k} \tanh \left(\frac{k H}{2}\right)}$ & $\sqrt{\frac{g^{\prime} H}{4}}$ & $\sqrt{\frac{g^{\prime}}{2 k}}$ \\
5.3 & Continously stratified & $\sqrt{\frac{g^{\prime}}{2 k} \tanh \left(\frac{k H}{2}\right) f_{i}\left(k \delta_{\rho}\right)}$ & $\sqrt{\frac{g^{\prime} H}{4} f_{i}\left(k \delta_{\rho}\right)}$ & $\sqrt{\frac{g^{\prime}}{2 k} f_{i}\left(k \delta_{\rho}\right)}$ \\
\hline
\end{tabular}

${ }_{651}$ In Table $1, k=2 \pi / \lambda_{w}$ is the wavenumber where $\lambda_{w}=2 L$ is the fun- 
damental wavelength in an enclosed domain of length $L, H$ is the depth, $g$ is gravity, $g^{\prime}=\frac{\rho-\rho_{0}}{\rho_{0}} g$ is the reduced gravity, $k H \rightarrow 0$ is the (hydrostatic) shallow-water limit, $k H \rightarrow \infty$ is the (nonhydrostatic) deep-water limit and $f_{i}\left(k \delta_{\rho}\right)=\left(1+k \delta_{\rho} / 2\right)^{-1}$ accounts for the effect of the finite-thickness interface (Kundu, 1990) where $\delta_{\rho}$ is the pycnocline thickness as discussed in Section 5.3 .

In Sections 5.1-5.3, the modeled wave speeds are determined by estimating the period of oscillation, $T_{\text {modeled }}$, from the simulation start time to the time of the next maximum in the free surface or isopycnal at $x=0$ and then calculating the wave speed based on $c_{\text {modeled }}=\omega_{\text {modeled }} / k=2 \pi /\left(k T_{\text {modeled }}\right)$.

In Table 1, the ratio of the hydrostatic wave speed to the theoretical (nonhydrostatic) wave speed for a free-surface seiche is given by

$$
\frac{c_{h}}{c}=\sqrt{\frac{k H}{\tanh (k H)}} .
$$

In this numerical experiment, we compare the wave speeds of the nonhydrostatic and hydrostatic models to the theoretical wave speed. Equation (36) shows that the hydrostatic model always overpredicts the wave speed except in the limit that $k H \rightarrow 0$. In this numerical experiment, we also compare depth-profiles of the horizontal and vertical velocity in the nonhydrostatic and hydrostatic models to linear theory. The analytical solutions for the horizontal and vertical velocity of a nonhydrostatic free-surface seiche are 
671

672

given by

$$
u=a g \frac{k}{\omega} \frac{\cosh (k(z+H))}{\cosh (k H)} \sin (k x) \sin (\omega t),
$$

$$
w=-a g \frac{k}{\omega} \frac{\sinh (k(z+H))}{\cosh (k H)} \cos (k x) \sin (\omega t),
$$

where $a$ is the initial amplitude of the free-surface seiche with analytical solution $\eta=a \cos (k x) \cos (\omega t)$. The corresponding analytical solutions for the horizontal and vertical velocity profiles of a hydrostatic free-surface seiche are given by

$$
u=a g \frac{k}{\omega} \sin (k x) \sin (\omega t)
$$

$$
w=-a g \frac{k}{\omega}(k(z+H)) \cos (k x) \sin (\omega t)
$$

which can be found by taking Equations (37) in the limit that $k H \rightarrow 0$.

The initial configuration of this numerical experiment is depicted in Figure $3(\mathrm{~A}, \mathrm{~B}, \mathrm{C})$, but with varying domain sizes, number of layers, and a smaller seiche amplitude ${ }^{8}$. We note that the densities of each layer do not necessarily need to change from one layer to the next as in the case of a free-surface seiche. The model is initialized with a cosine wave of the form $\eta=a \cos (\pi x / L)$ where the seiche amplitude is given by $a=0.1 \mathrm{~m}$ and the depth is given by $H=10 \mathrm{~m}$ on a grid with $N_{x}=64$ grid points in the horizontal and a varying number of layers in the vertical each with density $\rho_{0}=1000 \mathrm{~kg} \mathrm{~m}^{-3}$. To change the degree of nonhydrostasy, $k H$, the model is run with varying

${ }^{8}$ Note: The seiche amplitude is chosen to be small to minimize the free-surface nonlinearity, $\delta=a / H$, so that linear wave theory is valid. 
domain lengths while holding the depth fixed. The model is run with a freesurface Courant number $C=\frac{c \Delta t}{\Delta x}=1$ for 1.5 seiche periods, $t_{\max }=1.5 T$ where $T=2 \pi / \omega$.

The modeled wave speeds for the nonhydrostatic and hydrostatic models normalized by the theoretical wave speed agree with the relationships given by $c_{\mathrm{nh}} / c=1$ and Equation (36), respectively, as shown in Figure $4 \mathrm{~A}$. This indicates that the model achieves the correct dispersive behavior. When the nonhydrostasy parameter $k H$ is small, the hydrostatic and nonhydrostatic models are similar. However, when there is an appreciable degree of nonhydrostasy, the hydrostatic model overpredicts the true wave speed. Figure $4 \mathrm{~A}$ also demonstrates how the number of isopycnal layers affects the dispersive properties of the model. The effect of the nonhydrostatic pressure is to introduce depth-variability in the velocity field that decays like $k^{-1}$. Nonhydrostatic models with a limited number of layers underpredict the wave speeds, particularly for large values of $k H$. However, as layers are added to the nonhydrostatic model, the modeled wave speeds converge rapidly to the theory. For problems with low to moderate $k H$, a nonhydrostatic model with two layers is sufficient to accurately capture the dispersion (Stelling and Zijlema, 2003; Zijlema and Stelling, 2005). On the other hand, hydrostatic waves are non-dispersive and induce horizontal velocities that are depth-invariant (see Equation 38a). Consequently, the wave speeds in the hydrostatic model are uninfluenced by the number of layers.

The test case for the velocity profiles of a free-surface seiche uses the 
same parameters as above except with $H=16 \mathrm{~m}, L=10 \mathrm{~m}$ and $N_{\text {layers }}=20$ which are chosen so that the experiment is strongly nonhydrostatic. Figure 5 A shows the normalized horizontal velocity profiles for the nonhydrostatic and hydrostatic models at location $x=L / 2$ as compared to the theoretical profiles given in Equations (37a) and (38a), respectively, at time $t=T / 4$. Likewise, Figure $5 \mathrm{~B}$ shows the normalized vertical velocity profiles from the nonhydrostatic and hydrostatic model at location $x=0$ as compared to the theoretical profiles given in Equations (37b) and (38b), respectively, at time $t=T / 4$. In each case the model accurately reproduces the theoretical behavior. The most significant difference between the hydrostatic and nonhydrostatic velocity profiles is the depth-variation of the horizontal velocity. As seen in Figure $5 \mathrm{~A}$, the hydrostatic horizontal velocity profile does not vary with depth. In contrast, the nonhydrostatic horizontal velocity profile decays rapidly with depth from its maximum value at the free surface.

\subsection{Two-layer internal seiche}

In this test case, the (inviscid) isopycnal model is validated against linear theory describing internal gravity waves in a two-layer system. From Table 1 , the ratio of the hydrostatic wave speed to the theoretical wave speed for a two-layer seiche is given by

$$
\frac{c_{h}}{c}=\sqrt{\frac{k H / 2}{\tanh (k H / 2)}} .
$$


Equation (39) exhibits similar behavior to that of the free-surface seiche, (36), but differs in that the nonhydrostasy parameter is effectively halved for the internal seiche because the interface is located in the middle of water column which is effectively half as deep. In this test case, we also compare depth profiles of the horizontal and vertical velocity from the nonhydrostatic and hydrostatic models to theory which is computed with the first-mode linearized eigenfunction analysis of Fringer and Street (2003).

The initial configuration of this numerical experiment on the behavior of a two-layer internal seiche is shown in Figure 3 (D,E,F), but with varying domain sizes, number of layers, and a smaller constant seiche amplitude. The model is initialized with an internal cosine wave of the form $z_{1}=-H / 2+$ $a \cos (\pi x / L)$, where $a=0.1 \mathrm{~m}$ and $H=10 \mathrm{~m}$ on a grid with $N_{x}=64$ grid points in the horizontal and a varying number of layers in the vertical. We note that although the model may have more than two layers, the density only changes across the internal interface. The density in the upper half of the layers is $\rho_{1}=1000 \mathrm{~kg} \mathrm{~m}^{-3}$ and the density in the lower half of the layers is $\rho_{2}=1010 \mathrm{~kg} \mathrm{~m}^{-3}$. To change the degree of nonhydrostasy, $k H$, the model is again run with varying domain lengths as in the free-surface seiche test case.

As shown in Figure 4 B, the model achieves the correct dispersive behavior. The model of the two-layer internal seiche behaves in a very similar manner to the model of the free-surface seiche in terms of its performance as a function of $k H$ and the improvement of the dispersive properties as layers 
are added.

The test case for the velocity profile for the two-layer internal seiche uses the same parameters as above except with $H=16 \mathrm{~m}, L=10 \mathrm{~m}$ and $N_{\text {layers }}=40$ which are chosen so that the experiment is strongly nonhydrostatic. Figures $5 \mathrm{C}$ and $\mathrm{D}$ show the horizontal and vertical velocity profiles normalized by their respective maxima at locations $x=L / 2$ and $x=0$, respectively, at time $t=1 / 4 T$. In each case the model accurately reproduces the theoretical behavior. As seen in Figure $5 \mathrm{C}$, the hydrostatic horizontal velocity profile does not vary with depth above and below the interface. In contrast, the nonhydrostatic horizontal velocity profile decays rapidly away from its maximum value at the interface. Thus the ability of the nonhydrostatic model to accurately reproduce the modal solution, particularly one that is strongly nonhydrostatic, depends on the number of model layers used to represent the vertical variability of the solution even in regions of constant density.

\subsection{Continuously-stratified internal seiche}

In this test case, the (inviscid) isopycnal model is validated against linear theory describing internal gravity waves in a continuously-stratified system. From Table 1, the ratio of the hydrostatic wave speed to the theoretical wave speed, $c_{h} / c$, for a continuously-stratified internal seiche is identical to Equation (39). The theoretical wave speed for a continuously-stratified internal seiche is identical to the theoretical wave speed for a 2-layer internal seiche 
since the influence of the finite thickness interface (given in Table 1) is removed during the normalization. As in the previous test case, we compare depth-profiles of the horizontal and vertical velocity in the nonhydrostatic and hydrostatic models to theory from the first-mode linearized eigenfunction analysis of Fringer and Street (2003).

The initial configuration of this numerical experiment on the behavior of a continuously-stratified internal seiche is shown in Figure 3 (G,H,I), but with varying domain sizes, number of layers, and a smaller constant seiche amplitude. The model parameters are identical to those in the two-layer seiche case, with the exception of the density profile. The density in this case is given by

$$
\rho(x, z, t=0)=\rho_{0}+\frac{1}{2} \Delta \rho\left[1-\tanh \left(\frac{2 \tanh ^{-1} \alpha_{s}}{\delta_{\rho}}(z+H / 2-\xi)\right)\right]
$$

where the depth is $H=10 \mathrm{~m}, \alpha_{s}=0.99$ [see Fringer and Street (2003)], $\delta_{\rho}=1 \mathrm{~m}, \rho_{0}=1000 \mathrm{~kg} / \mathrm{m}^{3}$ and the density difference is given by $\Delta \rho / \rho_{0}=$ 0.01. The interface height $\xi=a_{i} \cos (k x)$ and $a_{i}=0.1 \mathrm{~m}$ as in the previous test case. The isopycnal layers are initialized to density contours that are linearly spaced in density space. Using this approach, the layers are localized at the stratified interface as shown in Figure $3 \mathrm{H}$. This approach resolves the stratification, but does not resolve the vertical variability caused by the nonhydrostatic effects (since no additional layers are present away from the interface). Thus we also consider a so-called "hybrid" approach, where layers 
are added to resolve the stratified interface as well as the velocity profile in regions above and below the interface as shown in Figure 3 I.

Figure $4 \mathrm{C}$ demonstrates that the model achieves the correct dispersive behavior. Interestingly, as demonstrated in Figure $4 \mathrm{C}$, adding layers to improve the representation of the stratification alone does not improve the dispersive properties. Additional layers are needed throughout the water column to improve the representation of the nonhydrostatic velocity profile with depth. The hybrid approach, which adds such layers, improves the dispersive characteristics.

The test case to compute the velocity profile of the continuously-stratified internal seiche uses the same parameters as the two-layer velocity profile test case (Section 5.2) except with 100 layers $^{9}$ in the hybrid configuration. Figures $5 \mathrm{E}$ and $\mathrm{F}$ show the horizontal and vertical velocity profiles normalized by their respective maxima at locations $x=L / 2$ and $x=0$, respectively, at time $t=1 / 4 T$. A similar test case for the velocity profile of an internal seiche is given in Fringer et al. (2006), where the velocity profiles are computed using the first-mode linearized eigenfunction analysis of Fringer and Street (2003). The eigenfunction analysis requires the wave frequency as input. In panels $\mathrm{E}$ and $\mathrm{F}$ of Figure 5, the wave frequency is computed both from the asymptotic theory in Table 1 (where $\omega=c k$ ) and from the model ( $\omega_{\text {modeled }}$ ). These are denoted as "NH Theory 1" and "NH Theory 2", respectively, in

\footnotetext{
${ }^{9}$ Note that we use 100 layers for graphical representation. The analytical solution may be well represented using fewer layers.
} 
Figures $5 \mathrm{E}$ and F. As expected, the modal solution using the wave frequency of the model more closely follows the modeled velocity profiles owing to errors in the asymptotic theory which is only valid in the limit of small $k \delta_{\rho}$. As seen in Figures $5 \mathrm{E}$ and $\mathrm{F}$, the hydrostatic and nonhydrostatic velocity profiles are well-represented using the model. In this case, the hydrostatic velocity profile has a smooth variation near the stratified interface (as in Figure 5 E) unlike the velocity profile for the two-layer stratification which is discontinuous across the interface (as in Figure $5 \mathrm{C}$ ).

In summary of the results in Sections 5.1-5.3, we find that

1. Nonhydrostatic dispersion gives rise to depth-dependence even in unstratified regions. Therefore, nonhydrostatic models require multiple layers to resolve both the vertical dependence of the velocity field and the dispersive properties, especially for large values of $k H$.

2. The horizontal velocity in unstratified regions does not vary with the vertical coordinate for hydrostatic models and thus adding layers in such regions does not affect the velocity profiles or the dispersive properties.

3. In weakly nonhydrostatic regimes for which $k H<1$, the depth-dependence and dispersion are minimal. Thus, in this regime, a model with a limited number of layers may suffice. 

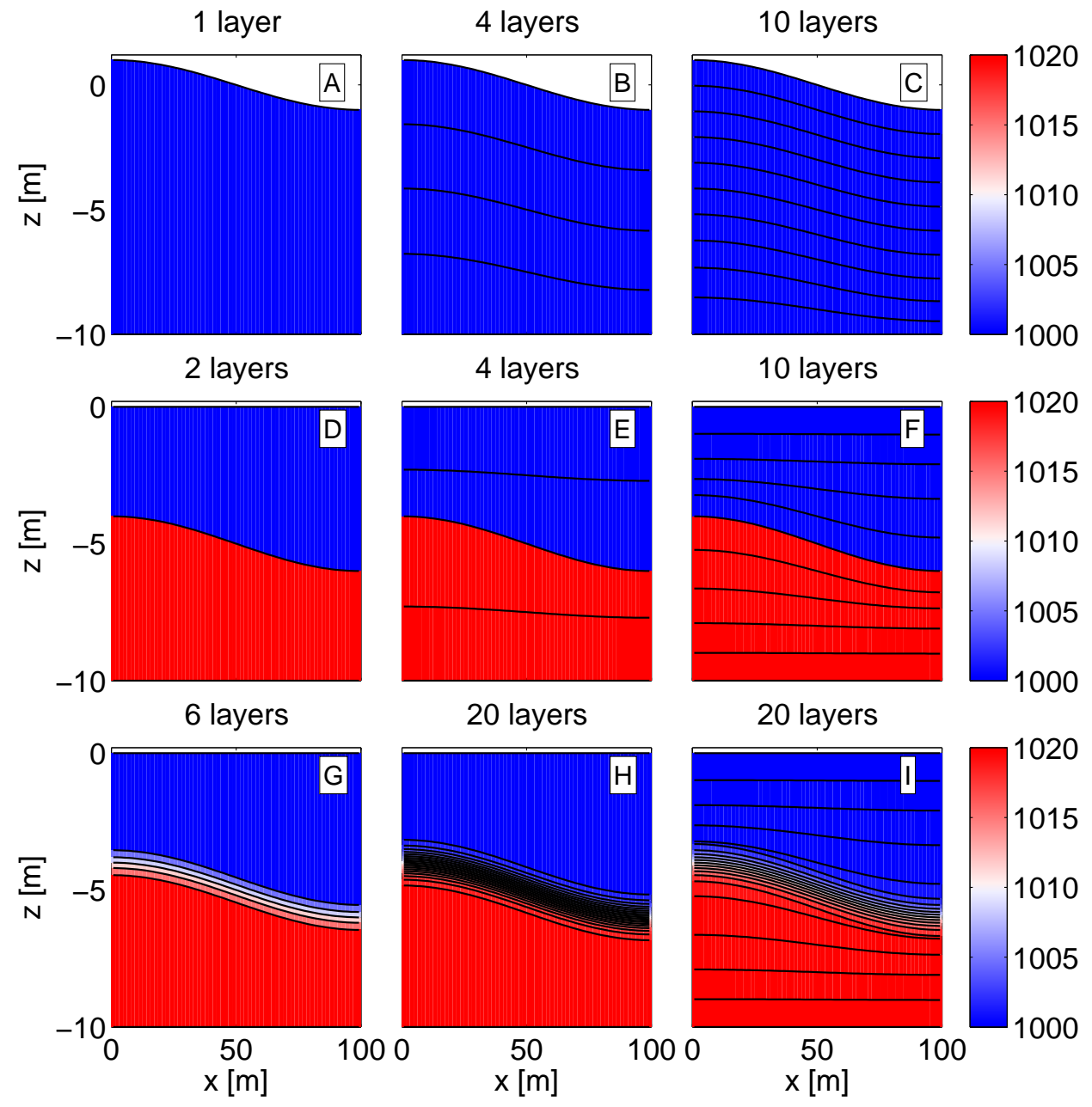

Figure 3: Initial density configurations $\left(\right.$ in $\mathrm{kg} \mathrm{m}^{-3}$ ) for a free-surface seiche (panels A,B,C), a 2-layer internal seiche (panels D,E,F), and a continuously-stratified internal seiche with a tanh density profile (panels G,H,I). In Sections 5.1-5.3, we use smaller wave amplitudes than shown here so that linear theory is valid. 

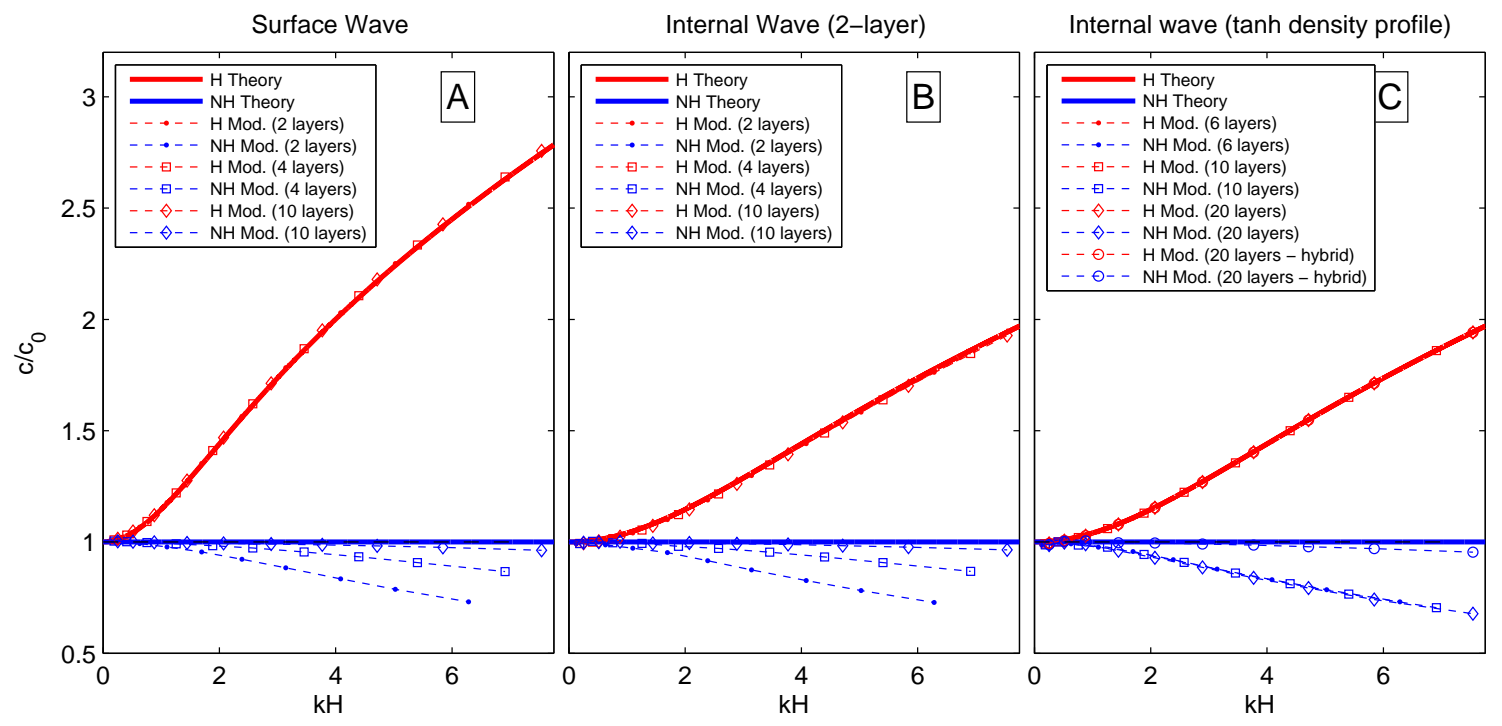

Figure 4: The dispersive characteristics of a free-surface seiche (Figure 3, top row), a 2-layer internal seiche (Figure 3, middle row), and a continuously stratified internal seiche (Figure 3, bottom row) in panels A,B, and C, respectively, for hydrostatic (red) and nonhydrostatic (blue) equations. The modeled and theoretical wave speeds are normalized by the theoretical wave speed, $c_{0}$, for each scenario. 

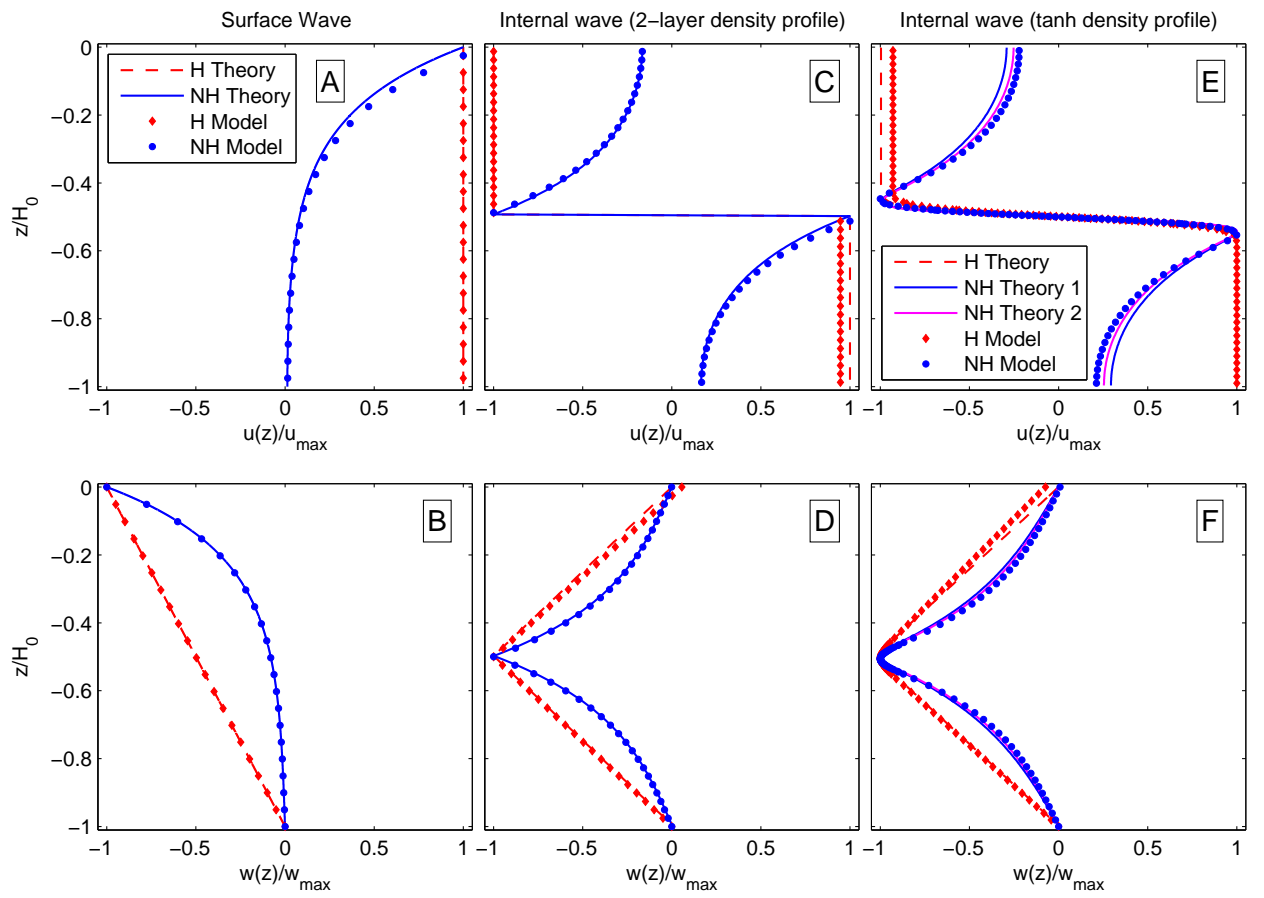

Figure 5: Normalized horizontal and vertical velocity profiles for a free-surface seiche (panels A and B), a two-layer internal seiche (panels C and D), and a continuously stratified internal seiche with a tanh density profile (panels $\mathrm{E}$ and F) for the hydrostatic (red) and nonhydrostatic (blue) models. 
5.4. Internal solitary waves: Comparison of isopycnal model to KdV theory

In this test case, we compare the widths of solitary waves formed using the current model to Korteweg and de-Vries (1895) (KdV) and extended KdV $(\mathrm{eKdV})$ theory. The well-known solitary-wave solution to the KdV equation is

$$
\xi=a_{0} \operatorname{sech}^{2}\left(\frac{x-c_{i} t}{L_{0}}\right)
$$

where the length scale of solitary waves in a two-layer system is given by

$$
L_{0}=\sqrt{\frac{4}{3} \frac{\left(h_{1} h_{2}\right)^{2}}{\left(h_{1}-h_{2}\right) a}},
$$

where $a$ is the amplitude of the solitary wave and $h_{1}$ and $h_{2}$ are the depths of the upper and lower layers, respectively (Bogucki and Garrett, 1993). After normalizing and rearranging, we can write Equation (42) as

$$
\frac{h_{1}}{L_{0}}=\frac{h_{1}}{h_{2}} \sqrt{\frac{3}{4}\left(1-\left(\frac{h_{1}}{h_{2}}\right)^{-1}\right) \frac{a}{h_{1}}},
$$

which relates the magnitude of nonlinearity $a / h_{1}$ to the wave aspect ratio $h_{1} / L_{0}$. The eKdV theory includes cubic nonlinearity and results in expressions similar to (41) and (42) although slightly more complicated as given in Helfrich and Melville (2006).

The simulations in this numerical experiment are performed in a domain of length $L_{d}=125 \mathrm{~km}$ and depth $H=2000 \mathrm{~m}$ with a two-layer stratification where $\rho_{1}=\rho_{0}=999.5 \mathrm{~kg} \mathrm{~m}^{-3}$ is the density of the upper layer, and $\rho_{2}=$ 
$\rho_{0}+\Delta \rho=1000.5 \mathrm{~kg} \mathrm{~m}^{-3}$ is the density of the lower layer. The simulations are performed in an enclosed domain with an initial isopycnal displacement of

$$
\xi(x, t=0)=z_{1}+h_{1}=-a_{i} \operatorname{sech}^{2}\left[\left(\frac{x}{L_{\rho}}\right)^{2}\right],
$$

with $h_{1}=250 \mathrm{~m}$ and $L_{\rho}=2 L_{0}$, and the model is run with varying initial amplitudes $a_{i}$. The initial wave of depression evolves rapidly into a solitary wave of width given closely by $L_{0}$ with a small train of trailing waves. The model is discretized with 2,4 , and 10 vertical layers each with $N_{x}=1000$ grid points in the horizontal. The model is inviscid and is run with a free-surface Courant number of 20 which corresponds to a time step of $17.85 \mathrm{~s}$.

Figure 6 shows $h_{1} / L_{0}$ vs. $a / h_{1}$ of the leading solitary waves (at $t=$ $16.29 \mathrm{hrs}$ ) for the isopycnal models with 2, 4 and 10 layers in panels A, B and C, respectively. The computed solitary wave amplitude $a$ and width $L_{0}$ are determined following the procedure used in Vitousek and Fringer (2011). The modeling results are compared to the $\mathrm{KdV}$ and $\mathrm{eKdV}$ theories. The main difference between the two theories is that the eKdV theory (which is valid for higher orders of nonlinearity) captures the tendency for solitary waves to broaden and develop flat crests as the waves become more nonlinear (Helfrich and Melville, 2006). Figure 6 demonstrates that for weakly nonlinear $\left(a / h_{1} \ll 1\right)$, weakly nonhydrostatic $\left(h_{1} / L_{0} \ll 1\right)$ waves, the model can reproduce solitary waves consistent with theory. However, for waves with an appreciable degree of nonlinearity $\left(a / h_{1}>0.15\right)$, the KdV theories 

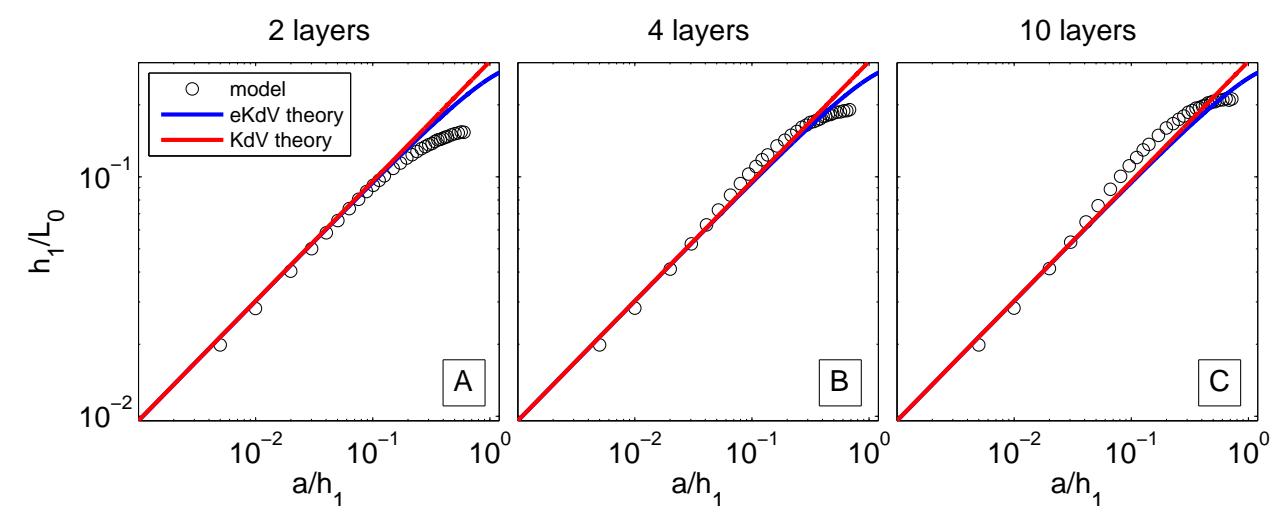

Figure 6: The wave aspect ratio $h_{1} / L_{0}$ vs. the magnitude of nonlinearity $a / h_{1}$ of the leading solitary waves computed by the isopycnal model at $t=81.27 \mathrm{hrs}$ compared to the $\mathrm{KdV}$ and $\mathrm{eKdV}$ theories.

are not valid; they underpredict the solitary wave widths (or equivalently overpredict $h_{1} / L_{0}$ relative to the modeling results). As shown in Figure 6, the eKdV theory captures the broadening of solitary waves (or equivalently decrease in $\left.h_{1} / L_{0}\right)$ as the nonlinearity increases (e.g. for $a / h_{1}>0.5$ ). However, the eKdV theory still underpredicts the solitary wave widths relative to the current model for high orders of nonlinearity. The slight mismatch between the model and the theoretical predictions is not due to model error but instead arises from the limited range of applicability of the weakly nonlinear, weakly nonhydrostatic KdV theory. Although in certain dynamical situations the model can revert to the simple KdV theory, it can also be applied to situations beyond weakly nonlinear, weakly nonhydrostatic regimes as demonstrated in the numerical experiments in the next section. 


\subsection{Internal solitary waves: Comparison of isopycnal model to z-level model}

In this test case, we compare formation of nonlinear internal solitary-like wave trains using the current isopycnal model to the nonhydrostatic SUNTANS $z$-level model (Fringer et al., 2006) in a two-dimensional $x$ - $z$ domain. The parameters for this test case follow the numerical experiment in Vitousek and Fringer (2011) using SUNTANS with 100 layers. This test case was chosen to approximate the evolution of solitary-like waves in the South China Sea following Zhang et al. (2011). The simulations are performed in a domain of length $L_{d}=300 \mathrm{~km}$ and depth $H=2000 \mathrm{~m}$ that is initialized with approximate two-layer stratification as an idealized representation of the South China Sea. The initial stratification is given by

$$
\rho(x, z, t=0)=\rho_{0}-\frac{1}{2} \Delta \rho \tanh \left[\frac{2 \tanh ^{-1} \alpha_{s}}{\delta_{\rho}}\left(z-\xi(x, t=0)+h_{1}\right)\right]
$$

where the upper-layer depth is $h_{1}=250 \mathrm{~m}, \alpha_{s}=0.99$ [see Fringer and Street (2003)], $\delta_{\rho}=200 \mathrm{~m}, \rho_{0}=1000 \mathrm{~kg} / \mathrm{m}^{3}$ and the density difference is given by $\Delta \rho / \rho_{0}=0.001$. The initial Gaussian of depression that evolves into a train of solitary-like waves is given by

$$
\xi(x, t=0)=-a_{i} \exp \left[-\left(\frac{x}{L_{\rho}}\right)^{2}\right]
$$

with $a_{i}=250 \mathrm{~m}$ and $L_{\rho}=15 \mathrm{~km}$. The boundary conditions are closed at $x=0$ and $x=L_{d}$ and the bottom at $z=-H$ is free-slip with a free surface at the upper boundary. The model is discretized with 2 and 10 
layers in the vertical. The layer heights and densities of the 10-layer model are initialized to follow contour lines of the density in Equation (45) which are linearly-spaced in density space. This numerical experiment is also run with 2 and 10 additional hybrid layers (distributed linearly in physical space in the upper and lower stratified layers) that are added to the simulation to improve the representation of the dispersive properties as discussed in Sections 5.1-5.3. The simulations are performed with $N_{x}=4800$ grid points in the horizontal. The model is run with a free-surface Courant number of 10 which corresponds to a time step of $17.85 \mathrm{~s}$. There is no viscosity or drag used in the isopycnal model, although the SUNTANS model uses (constant) viscosities of $\nu_{H}=0.1 \mathrm{~m}^{2} \mathrm{~s}^{-1}$ and $\nu_{V}=10^{-4} \mathrm{~m}^{2} \mathrm{~s}^{-1}$ to stabilize its central-differencing scheme for momentum advection. No scalar diffusivity is employed in the SUNTANS model.

The SUNTANS model requires approximately $2.0 \mathrm{~s}$ per time step $^{10}$ using 16 cores on four quad-core Opteron 2356 QC processors (Vitousek and Fringer, 2011). The 2-layer isopycnal model with the same horizontal resolution on the other hand requires approximately $0.15 \mathrm{~s}$ per time step using only one processor. This reduction in computational time and resources represents more than an order of magnitude improvement in efficiency. The 10-layer isopycnal model requires approximately 0.86 s per time step using

\footnotetext{
${ }^{10}$ We note that SUNTANS requires a time step of $\Delta t=5 \mathrm{~s}$ for these simulations which leads to a significant increase in computational effort compared to the time step of $\Delta t=$ 17.85 s needed in the isopycnal model.
} 
one processor and also represents a significant reduction in computational cost.

As shown in Figure 7, the initial Gaussian wave of depression steepens into a rank-ordered train of solitary waves. The 2- and 10-layer isopycnal models are shifted downward by $-700 \mathrm{~m}$ and $-350 \mathrm{~m}$ to allow comparison of the results. In Figure 7 , the $x$ - and $y$-axes are normalized by $L_{0}=1436 \mathrm{~m}$ and $a_{0}=220 \mathrm{~m}$, respectively, which represent the final solitary wave length and amplitude scales of the simulations presented in Vitousek and Fringer (2011). The time scale is normalized by $T_{s}=L_{0} / c_{1}=1028.7 \mathrm{~s}$, where $c_{1}=1.396 \mathrm{~m} \mathrm{~s}^{-1}$ is the speed of a mode- 1 wave given in Vitousek and Fringer (2011). Here, we adopt the same normalization to facilitate comparison with the results presented in Vitousek and Fringer (2011).

The speed of the internal waves varies slightly among the different models. The wave speed of the SUNTANS model is slightly reduced due to vertical numerically-induced scalar diffusion of density which is not present in the isopycnal model. We note that this scalar diffusion is purely numerical because no scalar diffusivity is employed. Numerically-induced scalar diffusion reduces the wave amplitude and thus the wave speed (which is weakly-dependent on amplitude) and also leads to thickening of the density contours in the wake of the leading wave in the wave train. The wave speed of the 10-layer isopycnal model is slightly slower than the 2-layer isopycnal model due to the finite-thickness of the interface in the 10-layer model which reduces the wave speed by a factor of $f_{i}\left(k \delta_{\rho}\right)$ as demonstrated in Table 1. 

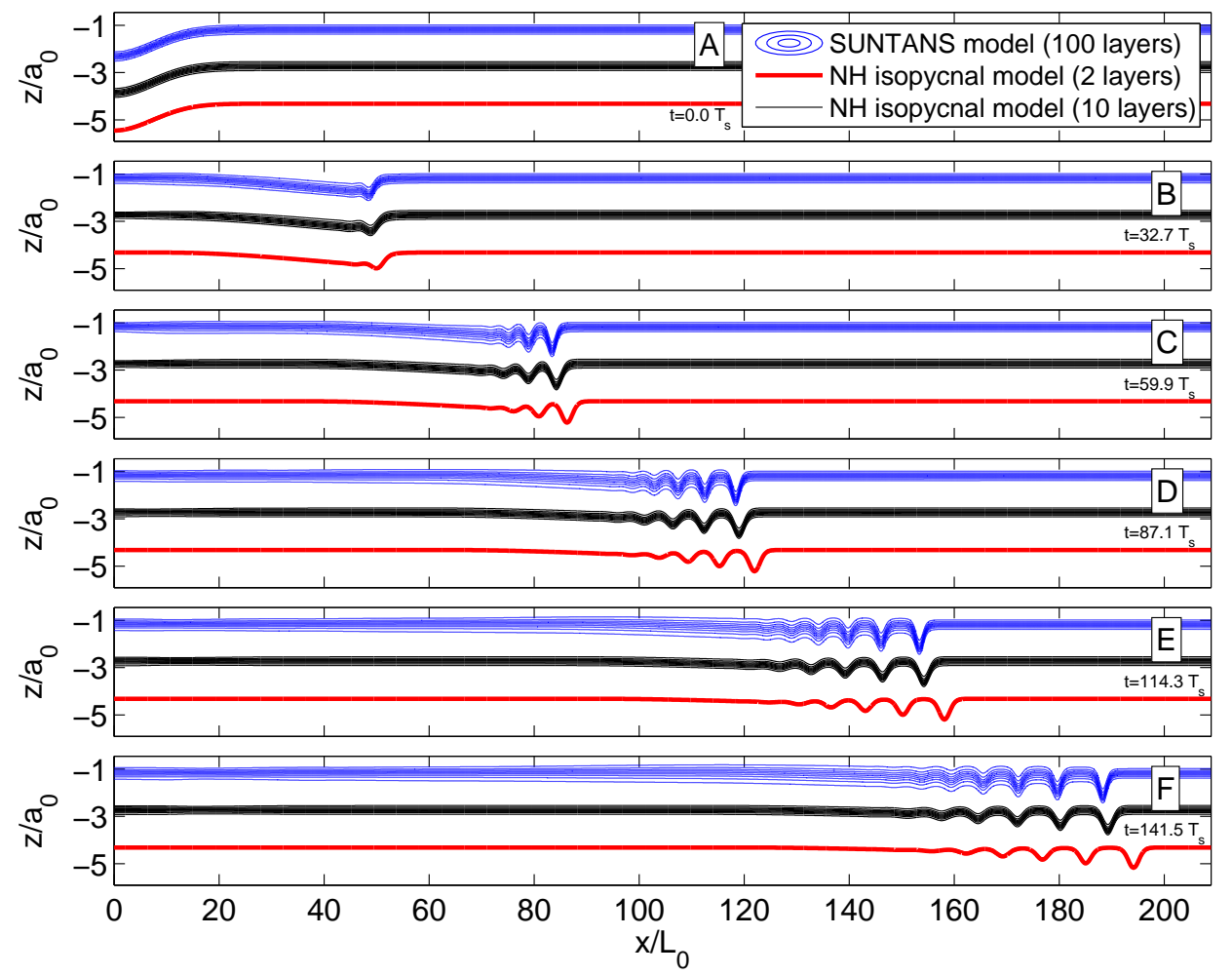

Figure 7: The formation of internal solitary waves generated from a Gaussian-shaped initial wave of depression computed by the current isopycnal model with 2 and 10 layers compared to the $z$-level SUNTANS model with 100 layers following the test case of Vitousek and Fringer (2011). The 2- and 10-layer isopycnal model results are shifted downward by $-700 \mathrm{~m}$ and $-350 \mathrm{~m}$, respectively, to allow comparison of the two models.

Overall, this numerical experiment demonstrates consistency between the number and widths of modeled solitary waves computed by the nonhydrostatic isopycnal model and the nonhydrostatic $z$-level model. The hydrostatic versions of SUNTANS and the current isopycnal model (not shown) are also remarkably similar.

Finally, we present a convergence study of the solitary wave widths as a function of grid resolution. This test case follows Vitousek and Fringer 
(2011) with the same simulation parameters as above but with a variable number of horizontal grid points, $N_{x}=[150,300,600,1200,2400,4800]$ and 2 and 10 additional "hybrid" layers (discussed in Section 5.3) to improve the dispersive properties. Figure 8 A shows the convergence study presented in Vitousek and Fringer (2011) using SUNTANS with 100 layers and Figure 8 B shows the convergence study of the current nonhydrostatic and hydrostatic isopycnal models with 2 and 10 layers. Figure 8 shows the normalized length scales $\left(L / L_{0}\right)$ of the leading solitary wave at the final time step, $t=40.4 \mathrm{hrs}$ $\left(t / T_{s}=141.5\right)$ as a function of grid lepticity $\lambda=\Delta x / h_{1}$. The procedure to determine solitary wave length scales is outlined in Vitousek and Fringer (2011). As demonstrated in Vitousek and Fringer (2011), the normalized length scales of the nonhydrostatic and hydrostatic models behave like

$$
L / L_{0}=\sqrt{1+K \lambda^{2}},
$$

and

$$
L_{h} / L_{0}=\lambda \sqrt{K},
$$

respectively, as a function of lepticity $\lambda$, where $K$ is a constant that depends on the numerical method and is obtained with a best-fit of the model results to expressions (47) and (48). Equations (47) and (48) arise from the secondorder accuracy of the numerical method. Numerical dispersion, which is proportional to the grid spacing squared, mimics physical dispersion and directly contributes to the widths of modeled solitary waves. Figure 8 B 
demonstrates that the isopycnal model has very similar convergence behavior to SUNTANS with respect to decreasing the grid spacing or grid lepticity. It is not surprising that the behavior of the two models is similar given that they employ similar numerical methods (and the same order of accuracy). However, the isopycnal model requires additional "hybrid" layers above and below the interface to better resolve the wave dispersion and thus the solitary wave length scales. Figure $8 \mathrm{~B}$ shows that adding layers above and below the stratification interface improves the modeled solitary wave widths. By adding 2 hybrid layers (indicated by +2 in Figure 8 ) the isopycnal model forms solitary waves that are approximately $10 \%$ larger than SUNTANS. By adding 10 hybrid layers (indicated by +10 in Figure 8) the isopycnal model forms solitary waves that are nearly identical to SUNTANS.

\subsection{Internal solitary waves: Comparison of isopycnal model to laboratory experiments}

This test case compares the formation of an internal solitary-like wave train using the current isopycnal model to the laboratory experiments in Horn et al. (2001). The laboratory experiments investigate the formation of solitary waves in an approximately two-layer density profile with an interface thickness of $\delta_{\rho}=0.01 \mathrm{~m}$ in an enclosed tank of depth $H=0.29 \mathrm{~m}$ and length $L=6 \mathrm{~m}$. The internal interface is initialized with a linear tilt which is given by

$$
\xi(x, t=0)=a_{i} \frac{x-L / 2}{L / 2},
$$



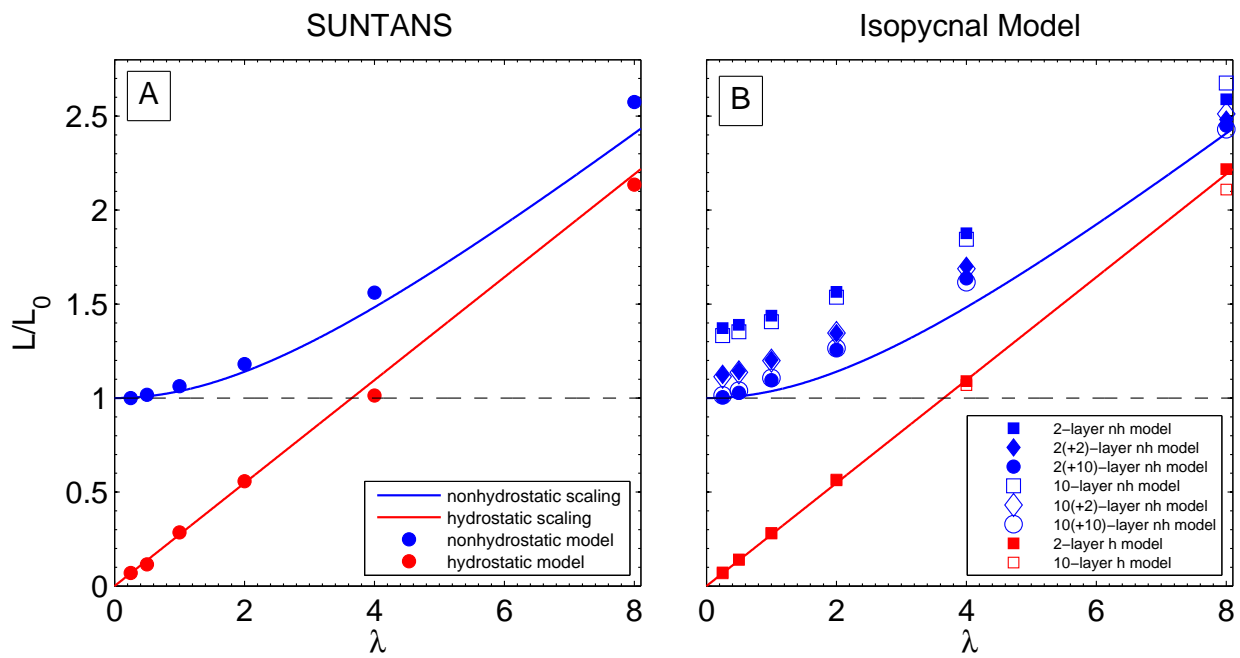

Figure 8: Convergence of leading solitary wave widths (at time $t=40.4 \mathrm{hrs}$ ) normalized by $L_{0}=1436 \mathrm{~m}$ as a function of the grid lepticity $\lambda=\Delta x / h_{1}$. Panel A shows the results from the SUNTANS model following Vitousek and Fringer (2011). Panel B shows the results from the isopycnal model with different numbers of layers. For reference the theoretical convergence relationships for the nonhydrostatic (blue line) and hydrostatic models (red line) given in Equations (47) and (48), respectively, are shown. 


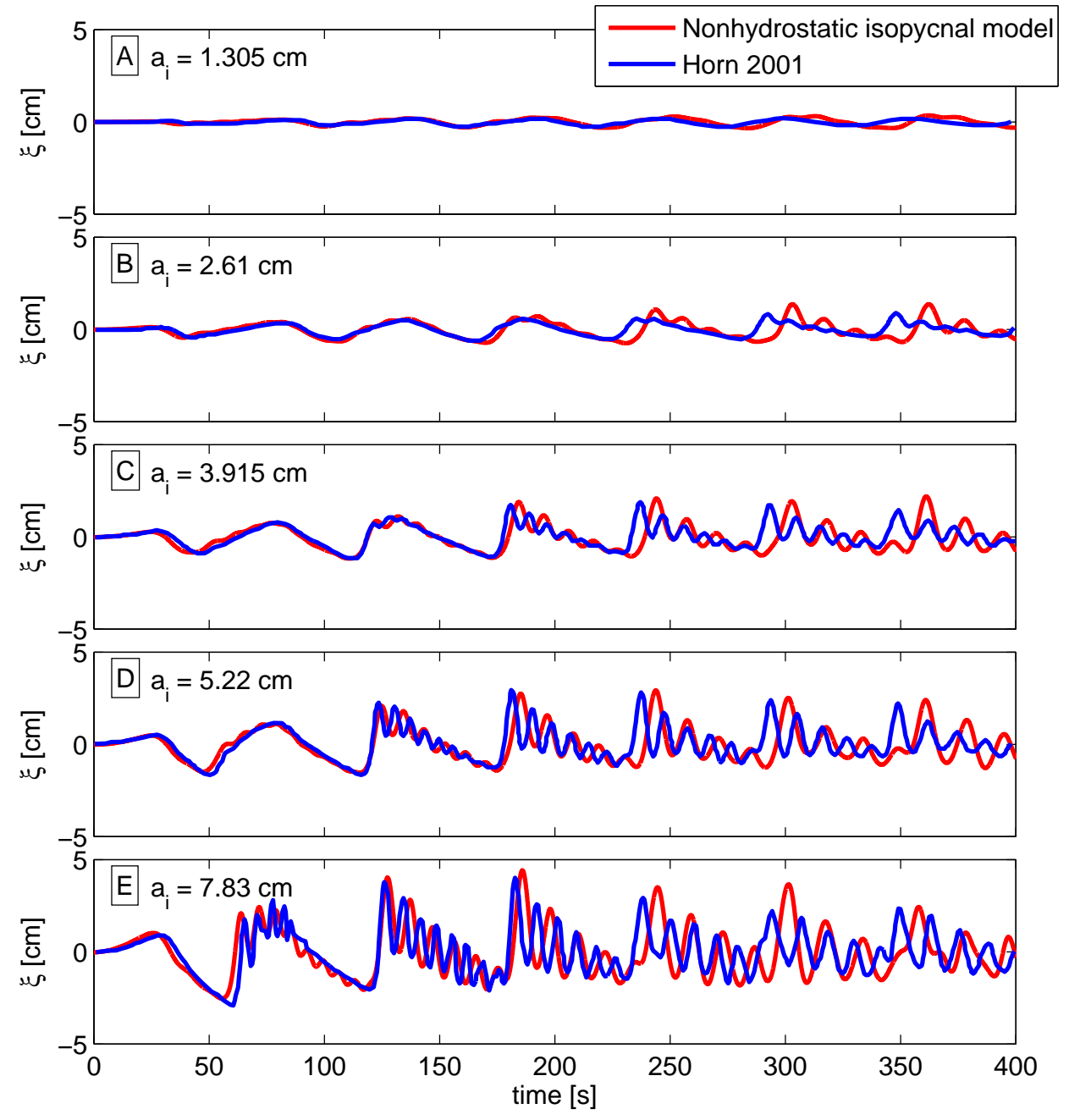

Figure 9: Comparison of nonhydrostatic model-predicted and measured (from Horn et al. (2001)) interface displacement $(\xi)$ time series at $x=L / 2$. 


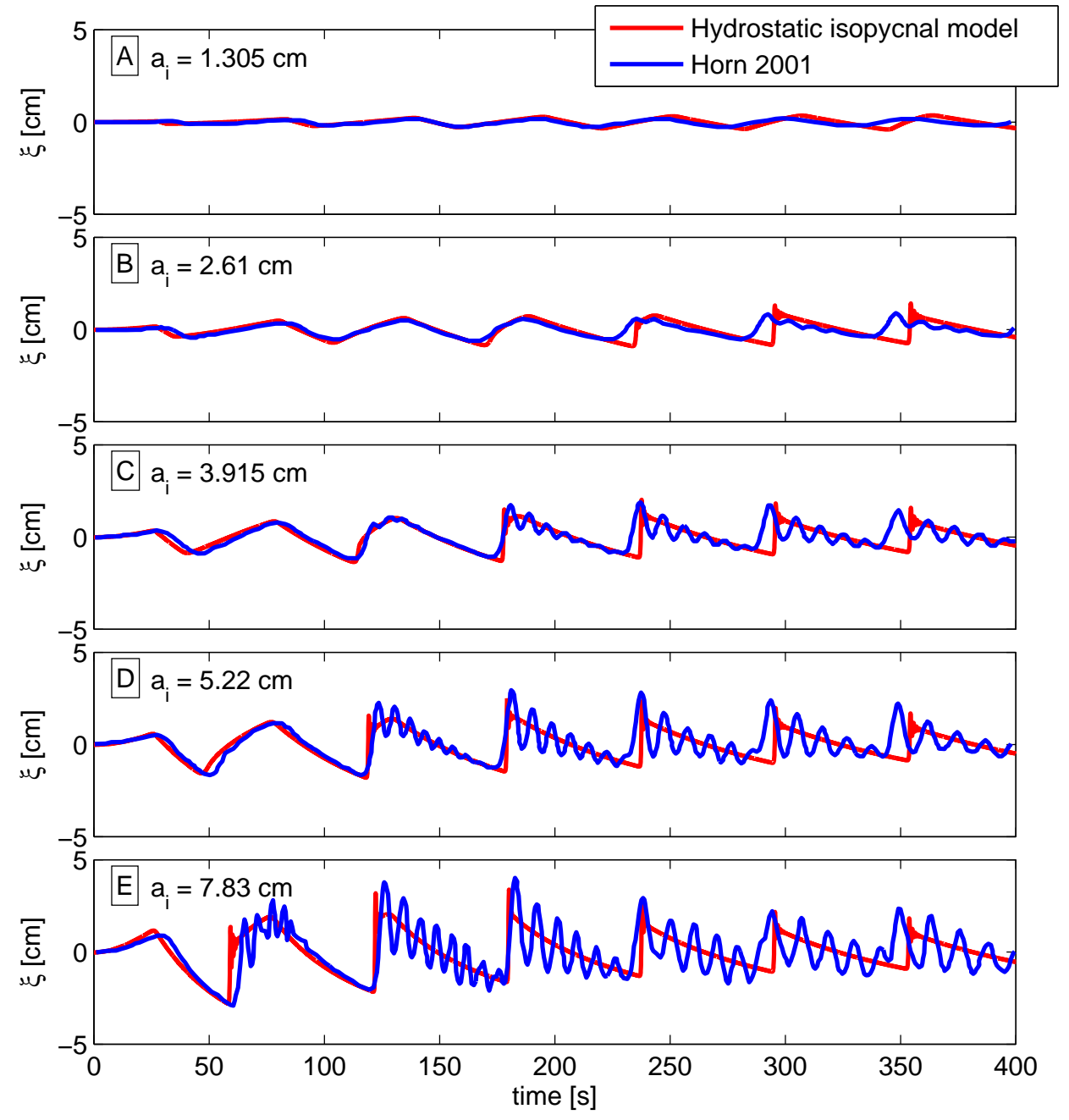

Figure 10: Comparison of hydrostatic model-predicted and measured (from Horn et al. (2001)) interface displacement $(\xi)$ time series at $x=L / 2$. The hydrostatic model forms steep bores instead of trains of solitary waves as in Figure 9. 
where the wave amplitude is given by $a_{i}=[0.01305,0.0261,0.03915,0.0522$, $0.0783 \mathrm{~m}$. The model of the laboratory experiments is run with 2 layers and $N_{x}=256$. The density difference in the laboratory experiments is approximately $\frac{\Delta \rho}{\rho_{0}}=0.02 \pm 0.002$. The model is run with a slightly lower density difference of $\frac{\Delta \rho}{\rho_{0}}=0.0175$ because the finite-thickness interface in the laboratory experiment reduces the wave speed slightly when compared to the two-layer configuration used in the model with (still-water) layer heights $h_{1}=0.203 \mathrm{~m}$ and $h_{2}=0.087 \mathrm{~m}$. The model is run with a free-surface Courant number $C=4$ for a total time of $400 \mathrm{~s}$. The model uses a drag coefficient $C_{d}=0.0175$ and horizontal and vertical eddy-viscosities of $\nu_{H}=1 \times 10^{-6} \mathrm{~m}^{2} \mathrm{~s}^{-1}$ and $\nu_{v}=1 \times 10^{-6} \mathrm{~m}^{2} \mathrm{~s}^{-1}$, respectively. These parameters are chosen to fit the amplitude of the solitary waves formed during the laboratory experiment. Figure 9 shows the results of the model-predicted time series of the interface displacements (located at the center of the tank $x=L / 2$ ) compared to the laboratory experiments of Horn et al. (2001). Panels A,B,C,D,E of Figure 9 correspond to the increasing initial interface amplitudes of $a_{i}=[0.01305$, $0.0261,0.03915,0.0522,0.0783] \mathrm{m}$, respectively. The agreement between the model and the laboratory experiments is excellent for the first half of the experiment $(t<200 \mathrm{~s})$. However, for the second half of the experiment there is some mismatch in the phase and amplitude of the waves. Over the course of a laboratory experiment Horn et al. (2001) reported "a gradual thickening of the density interface over the set, typically from approximately $1 \mathrm{~cm}$ to 2 $\mathrm{cm}$ ". The finite-thickness interface and thickening are partly responsible for 
the mismatch with the model (which has a sharp interface and no interface thickening). Additionally, the model appears to form slightly wider solitary waves than the laboratory experiment. This indicates that the dispersive properties of the waves are not captured exactly in the model because of the two-layer approximation. Overall, however, the model adequately captures the formation of solitary waves in the laboratory experiment.

Figure 10 shows the same numerical experiment as Figure 9 except using the developed isopycnal model in hydrostatic mode. The hydrostatic model in Figure 10 forms steep bores as opposed to trains of solitary waves as in Figure 9. This is particularly visible for the experiments with larger initial amplitudes. Although nonhydrostatic dispersion does influence the wave speed, the effect is relatively weak. Consequently, the wave speed of the nonhydrostatic and hydrostatic models are consistent. This is evidenced by the remarkably similar timing of the modeled hydrostatic wave fronts compared to the laboratory experiments. Therefore, a hydrostatic model may suffice for predicting the arrival time of the initial wave fronts. However, the significant difference between the hydrostatic and nonhydrostatic models is the form of the trailing waves. The hydrostatic model in Figure 10 has a tendency to form narrow, numerically-induced ${ }^{11}$ solitary waves on each wave front. These numerically-induced solitary waves are, in this case, much narrower than the waves in the laboratory data because the numerical dispersion

\footnotetext{
${ }^{11}$ generated by dispersive (odd-order) truncation error
} 
is much smaller than (the unmodeled) physical dispersion due to the high grid resolution. The numerical dispersion is smaller than physical dispersion because the grid lepticity, $\lambda=\frac{\Delta x}{h_{1}}=0.1155$ is less than unity (Vitousek and Fringer, 2011). In summary, these numerical experiments demonstrate the necessity of using nonhydrostatic models when simulating nonlinear internal solitary waves like those in the experiments of Horn et al. (2001).

\subsection{Internal solitary waves: Internal wave generation in the South China} Sea

In this numerical experiment, we investigate the capability to simulate solitary wave formation in realistic physical domains that are typically simulated using nonhydrostatic $z$ - or $\sigma$-coordinate models. The prospect for simulating complex internal wave generation with a limited vertical layer structure seems possible due to the work of Li and Farmer (2011) who successfully applied a fully nonlinear two-layer model to predict the evolution of the internal tide in the South China Sea.

In this test case, we follow the modeling results of Buijsman et al. (2010) who studied the generation and evolution of nonlinear internal waves in the South China Sea with a $\sigma$-coordinate model using 60 layers. The model is run with bathymetry that approximates the eastern ridge of the Luzon Strait with a height of $a_{b}=2600 \mathrm{~m}$ in a depth of $H_{0}=3000 \mathrm{~m}$. Following Buijsman 


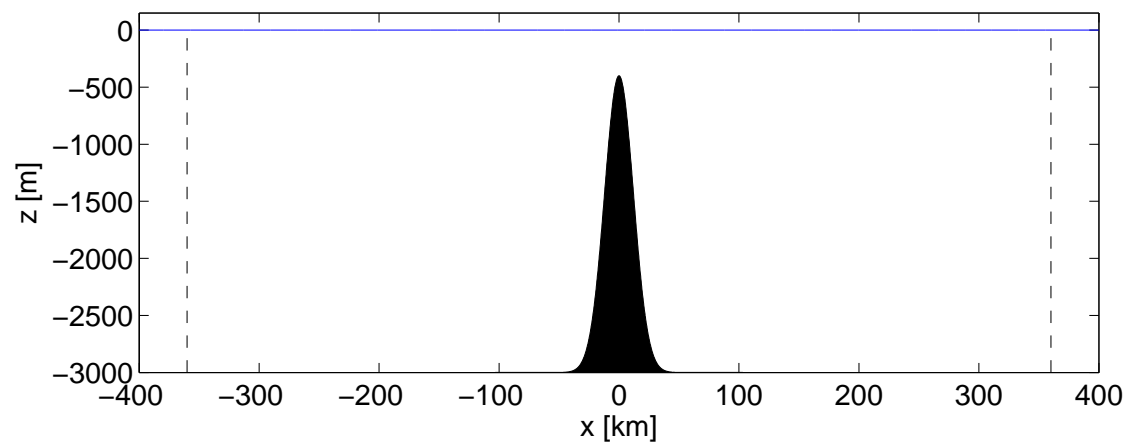

Figure 11: The model domain and bathymetry used in the South China Sea test case following Buijsman et al. (2010). The black filled area represents the bathymetry, the blue line represents the free surface, and the black dashed lines represent the locations of the sponge layers.

et al. (2010), the depth profile is given by

$$
H=H_{0}-a_{b} \exp \left(-\frac{x^{2}}{2 L_{b}^{2}}\right)
$$

where the bathymetry length scale $L_{b}=12 \mathrm{~km}$. The model domain and bathymetry are shown in Figure 11.

A least squares fit to the density profile in Buijsman et al. (2010) gives

$$
\rho(z)=\rho_{0}+\Delta \rho\left(1-\exp \left(z / h_{0}\right)^{p}+s_{1} z+s_{2} z^{2}\right)
$$

where $\rho_{0}=1024.75 \mathrm{~kg} \mathrm{~m}^{-3}, \Delta \rho=5.22 \mathrm{~kg} \mathrm{~m}^{-3}, h_{0}=224 \mathrm{~m}, p=1.15$, $s_{1}=-0.00018 \mathrm{~m}^{-1}$, and $s_{2}=-3.64 \times 10^{-8} \mathrm{~m}^{-2}$ are the best-fit parameters. Internal waves are generated from tidal flow in the Luzon Strait over the bathymetric ridge. To capture this in the model, the boundary is forced at 
both sides of the domain by

$$
u_{\mathrm{bc}}=u_{0} \sin \left(\omega_{\mathrm{bc}} t\right) \text {, }
$$

where $u_{0}=0.134 \mathrm{~m} \mathrm{~s}^{-1}$ and $\omega_{\mathrm{bc}}=1.41 \times 10^{-4} \mathrm{~s}^{-1}$ as in case R1 in Buijsman et al. (2010).

The model is run in hydrostatic and nonhydrostatic mode with 2 and 10 layers. The initial layer configurations are set to best match the weakly nonlinear and nonhydrostatic behavior of solitary waves in the stratification given by Equation (51). The still-water interface depths in the 10-layer isopycnal model are arbitrarily set to $z=-[0,50,100,250,460,750,1000,1500,2000,2500,3000] \mathrm{m}$ with density values computed at the vertical center of each layer using Equation (51). Following the procedure described in Appendix F, the still-water internal interface for the 2-layer model is set to $z=-460 \mathrm{~m}$ and the upper- and lower-layer densities are set to $\rho_{1}=1028 \mathrm{~kg} \mathrm{~m}^{-3}$ and $\rho_{2}=1030.1967 \mathrm{~kg} \mathrm{~m}^{-3}$, respectively, in order to match the mode- 1 wave speed of the 10-layer model.

To properly simulate the generation and evolution of internal waves in the South China Sea, the model is run with a domain length of $L=800 \mathrm{~km}$ and $N=1750$ grid points so that $\Delta x \approx h_{1}=460 \mathrm{~m}$ and the grid lepticity $\lambda=\frac{\Delta x}{h_{1}} \approx 1$ so that the numerical dispersion is relatively small according to Vitousek and Fringer (2011).

The model is run with a horizontal eddy-viscosity of $\nu_{H}=100 \mathrm{~m}^{2} \mathrm{~s}^{-1}$ and 
a vertical eddy-viscosity of $\nu_{v}=1 \times 10^{-4} \mathrm{~m}^{2} \mathrm{~s}^{-1}$ to characterize unresolved mixing of momentum due to turbulence in the presence of internal waves and a drag coefficient of $C_{d}=0.0025$. The model uses the QUICK (upwindbiased, 3rd-order spatially accurate) advection scheme of Leonard (1979) to reduce spurious, numerically-induced oscillations associated with centraldifferencing methods. The model is run with a free-surface Courant number of $C=10$ which gives a time step of $\Delta t=26.5 \mathrm{~s}$. The simulation starts from rest and runs for 10 tidal periods. To minimize internal wave reflection from the boundaries, the model uses sponge layers following Zhang et al. (2011) on each boundary of the domain. These are implemented by adding a term to the right-hand side of the horizontal momentum equation of the form

$$
S(x, z, t)=-\frac{u(x, z, t)-u_{\mathrm{bc}}(x, z, t)}{\tau_{s}} s(r)
$$

where $s(r)=\exp \left(-4 r / L_{s}\right)$ causes the influence of the sponge layer to decay over the distance $L_{s}=L / 20$ where $r$ is the distance to the domain boundary and $\tau_{s}=65 \mathrm{~s}$ is the damping time scale.

Figure 12 shows the generation and evolution of internal solitary waves in the nonhydrostatic 2-layer vs. 10-layer model. Panels A-F depict the interface displacement of the isopycnal initially located at $z=-h_{1}=-460 \mathrm{~m}$ at time intervals of $0.75 T_{\mathrm{bc}}$ where $T_{\mathrm{bc}}=2 \pi / \omega_{\mathrm{bc}}$. The 2 - and 10-layer models form trains of nonlinear internal solitary waves with nearly identical wave speeds, although the amplitude, length scales, and number of solitary waves 
differ between the models. The two-layer model only supports mode-1 waves whereas the 10-layer model supports higher modes. Consequently, in the 2layer model, all of the barotropic-to-baroclinic energy is converted to mode-1 waves. In the 10-layer model, energy is converted to higher-mode waves, the effects of which are clearly visible near the ridge. This results in larger amplitude solitary waves in the 2-layer model relative to the 10-layer model. Additionally, the solitary wave widths in the 2-layer model are slightly larger than the 10-layer model due to unresolved dispersion in models with limited vertical resolution (discussed in Section 5.5) and the larger dispersion coefficient, $\epsilon$, in the 2-layer model relative to the 10-layer model (see Appendix F).

Figure 13 shows the same test case as in Figure 12 except using the hydrostatic model. Instead of trains of nonlinear solitary waves, the 2- and 10-layer models form steep bores with nearly identical wave speeds. The wave amplitudes differ slightly between the 2- and 10-layer model, as in the nonhydrostatic simulations, due the energy conversion to the higher-mode waves supported by the 10-layer model. Comparing Figure 13 to Figure 12, the 2- and 10-layer hydrostatic models are more similar to each other than the 2- and 10-layer nonhydrostatic models. This result is expected because of the conclusions presented in Sections 5.1-5.3. Specifically, there is no difference in the dispersive properties of the hydrostatic models, i.e. they are both physically nondispersive. Consequently, there is no depth variability that must be resolved with additional vertical layers in order to improve the 

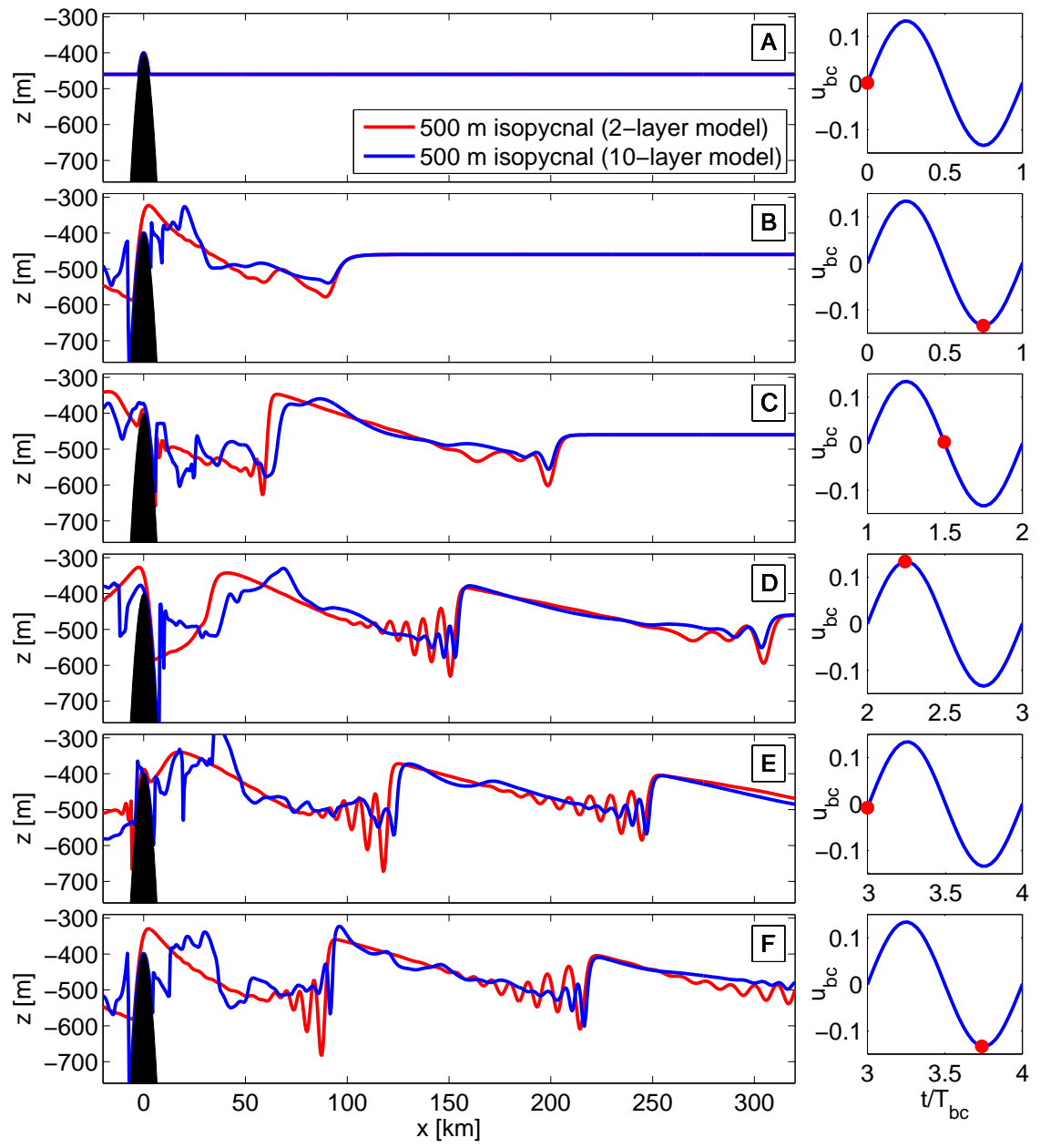

Figure 12: Comparison of internal wave generation in the nonhydrostatic 2-layer (red) and 10-layer (blue) model at time intervals of $0.75 T_{b c}$. Both models form trains of nonlinear internal solitary waves. The two-layer model only supports mode-1 waves whereas the 10-layer model supports higher modes, the effects of which are visible near the ridge. 
model's performance with regard to the mode-1 wave speed and shape.

The model results presented in Figures 12 and 13 are used to compute the baroclinic energy flux associated with internal waves which is given by

$$
F=\sum_{k} u_{k}^{\prime} M_{k}^{\prime} h_{k}
$$

where $u_{k}^{\prime}=u_{k}-\frac{1}{\sum_{k} h_{k}} \sum_{k} u_{k} h_{k}$ is the baroclinic perturbation velocity and $M_{k}^{\prime}=\sum_{m=2}^{k}\left(\rho_{m}-\rho_{m-1}\right) g\left(z_{m-1}-\left(z_{0}\right)_{m-1}\right)$ is the perturbation baroclinic Montgomery potential, and $\left(z_{0}\right)_{m}$ represents the interface location for the $m$-th still-water isopycnal (Simmons et al., 2004; Miao et al., 2011). We note that the nonhydrostatic contributions to the energy flux, $F_{\mathrm{nh}}=\sum_{k} u_{k}^{\prime} q_{k} h_{k}$, are negligible for the simulations presented here. The results for the maximum, tidally-averaged energy flux, $F_{\max }$, and the spatial location, $x_{\max }$, where the maximum occurs, are given in Table 2. $F_{\max }$ is obtained by applying a tidally-averaging filter, a moving average of size $T_{b c}$, over the simulation time series and averaging the filtered value for the last 7 tidal periods (after 3 tidal periods of spin up). As indicated in Table 2, the maximum energy flux, $F_{\max }$, for the 2-layer hydrostatic and nonhydrostatic models are nearly identical which indicates the relative unimportance of nonhydrostatic effects as far as the large-scale energetics is concerned. However, nonhydrostatic effects substantially alter the amplitude and distribution of the solitary waves. The 10-layer hydrostatic and nonhydrostatic models are also very similar with approximately $5-10 \%$ relative difference in energy flux between the hy- 

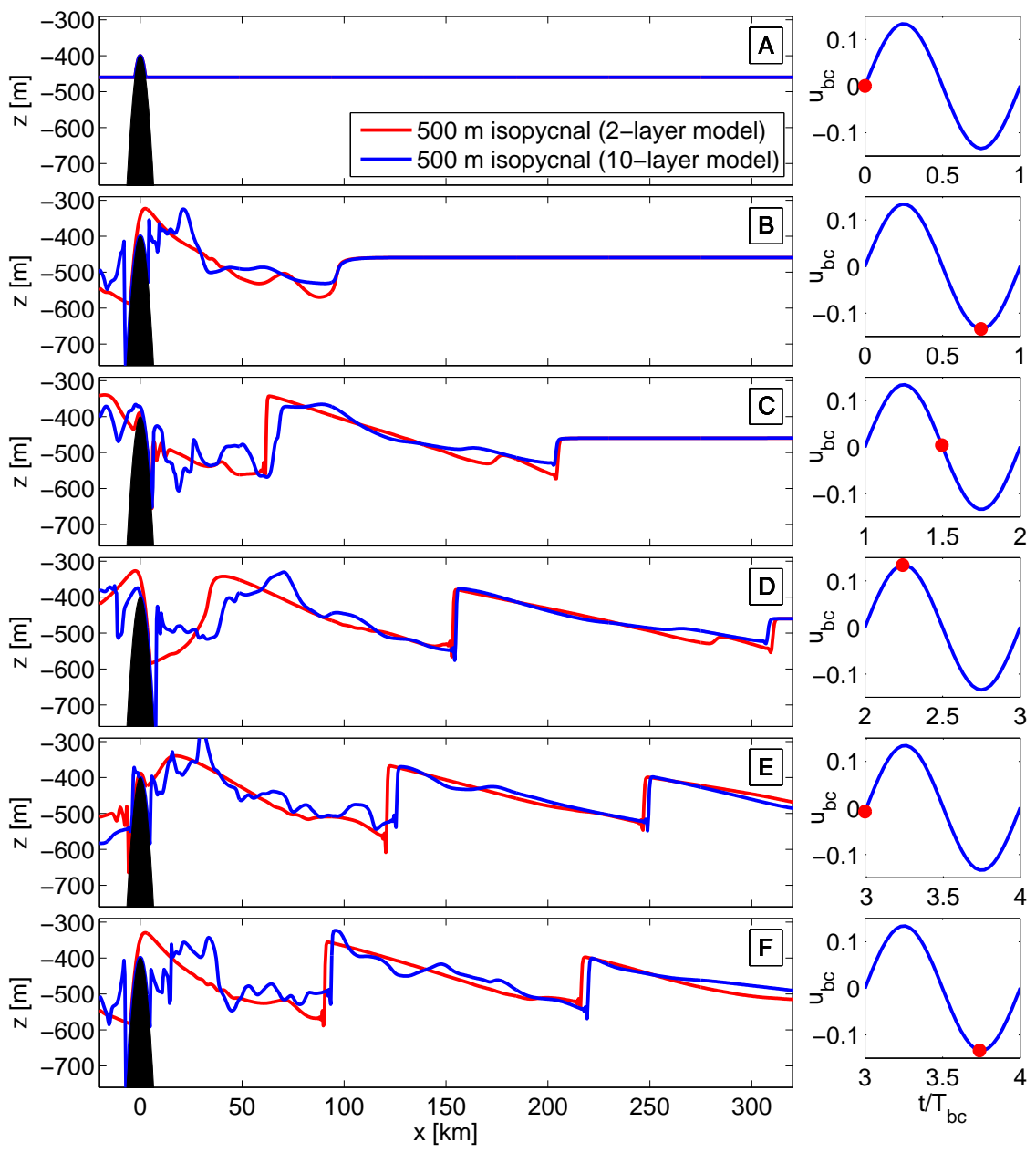

Figure 13: Comparison of internal wave generation in the hydrostatic 2-layer (red) and 10-layer (blue) model at time intervals of $0.75 T_{b c}$. Instead of trains of nonlinear internal solitary waves of, both models form bores. The two-layer model only supports mode- 1 waves whereas the 10-layer model supports higher modes, the effects of which are visible near the ridge. 
drostatic and nonhydrostatic models. The locations of the maximum energy flux also show good agreement — they differ in location by a maximum of 14 grid points of the total 1750 . The values for the energy flux reported here are qualitatively similar to the values in Warn-Varnas et al. $(2010)^{12}$.

Table 2: Maximum energy flux $\left(F_{\max }\right)$ and its location $x_{0}$ for the South China Sea test case.

\begin{tabular}{clcc}
\hline \# of layers & nonhydrostatic mode & $F_{\max }\left[\mathrm{kW} \mathrm{m}^{-1}\right]$ & $x_{\max }[\mathrm{km}]$ \\
\hline 2 & hydrostatic & 328.8 & 29.7 \\
2 & nonhydrostatic & 328.4 & 30.2 \\
10 & hydrostatic & 354.4 & 31.1 \\
10 & nonhydrostatic & 365.6 & 36.1 \\
\hline
\end{tabular}

As mentioned earlier, the main difference between the internal waves in the 2-layer vs multilayer systems is the presence of higher modes. Figure 14 shows a Hovmöller diagram for the interface displacement of the isopycnal initially located at $z=-h_{1}=-460 \mathrm{~m}$ for the 2- and 10-layers in Panels A and B, respectively. Hovmöller or "trough-and-ridge" diagrams (Hovmöller, 1949) depict a particular quantity as a function of $x$ and $t$. These diagrams are informative because lines on a Hovmöller diagram correspond to wave characteristics. Figure 14 shows the tidal velocity (black solid line whose magnitude is shown on the top $x$-axis), and the first- (black, dashed line) and second-mode (black, dotted line) wave speeds which are given by $c_{1}=2.86 \mathrm{~m} \mathrm{~s}^{-1}$ and $c_{2}=1.52 \mathrm{~m} \mathrm{~s}^{-1}$, respectively. Figure 14 A shows

\footnotetext{
${ }^{12}$ Values for the energy flux are not reported in Buijsman et al. (2010).
} 

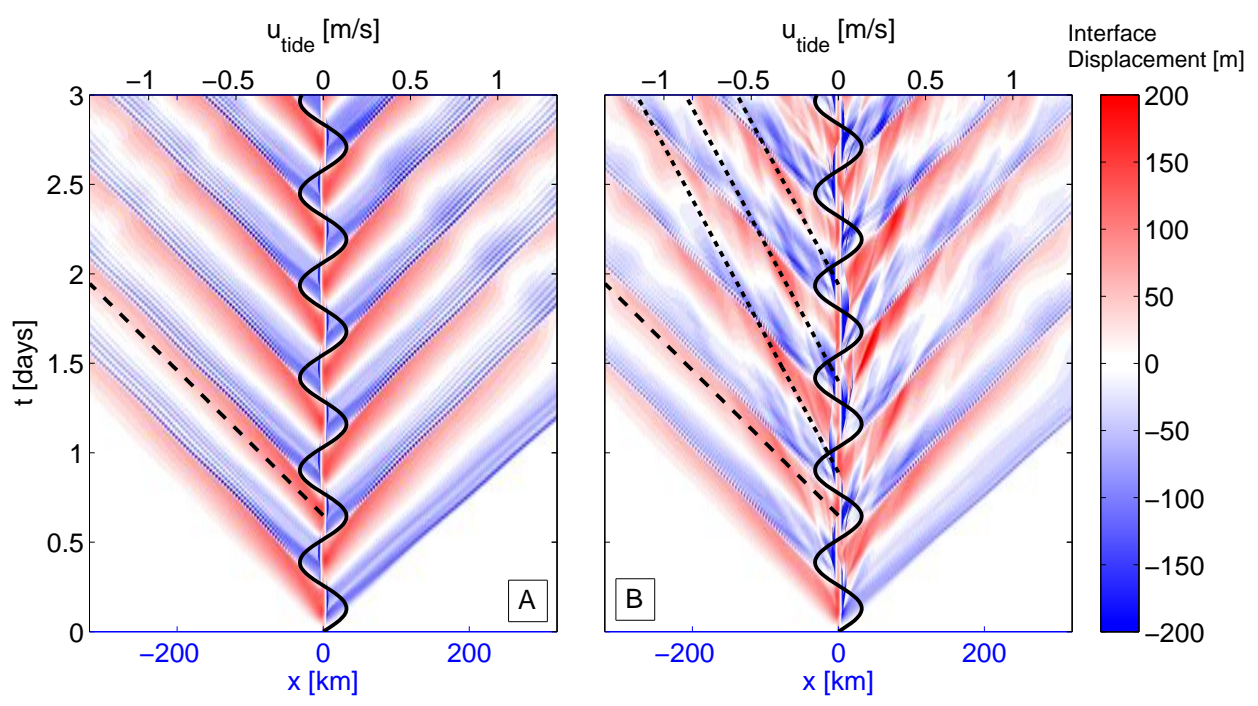

Figure 14: Hovmöller diagram ( $x$ vs. $t$ ) for the South China Sea test cases with 2- and 10-layers in Panels A and B, respectively. These diagrams show the internal interface displacement of the isopycnal initially located at $-460 \mathrm{~m}$. Shown for reference are tidal velocity (black, solid line), and the first- (black, dashed line) and second-mode (black, dotted line).

how the 2-layer model only generates mode-1 characteristics. The 10-layer model, on the other hand, generates mode-1 and higher-mode characteristics that match both the mode- 1 and mode- 2 characteristics in Figure 14 B. The Hovmöller diagram shown in Figure $14 \mathrm{~B}$ is qualitatively similar to the Hovmöller diagram for the $\sigma$-coordinate model results presented in Figure 5 of Buijsman et al. (2010), with respect to the solitary and higher-mode wave generation.

Overall, the results demonstrate good performance of isopycnal-coordinate models with limited vertical resolution to represent both the small-scale dynamics (the formation of nonlinear internal solitary waves) and the large-scale dynamics (the baroclinic energy flux). 


\subsection{Internal wave beams}

The last test case is the formation of internal wave beams generated from oscillatory flow over a Gaussian sill. The primary goal of the developed model is the treatment of internal waves with a reduced number of vertical layers. However, in this test case, we demonstrate that the current isopycnal model is also applicable to modeling continuously stratified environments and internal wave beams which require $\mathcal{O}(10-100)$ layers to be well resolved in the vertical.

A fluid with constant stratification of

$$
N^{2}=-\frac{g}{\rho_{0}} \frac{\partial \rho}{\partial z}
$$

produces internal wave beams radiating with a constant slope given by

$$
\frac{d z}{d x}=\tan (\varphi)=\sqrt{\frac{\omega^{2}-f^{2}}{N^{2}-\omega^{2}}},
$$

[see e.g. Kundu (1990)], where $\omega$ is the forcing frequency and $f$ is the Coriolis parameter.

The purpose of this numerical experiment is to demonstrate that the model is able to correctly capture the internal wave beam angle and to demonstrate the difference between the nonhydrostatic and hydrostatic beam angles. The nonhydrostatic internal wave beam angle in the absence of the 
1190

Coriolis force $(f=0)$ is given by

$$
\varphi=\tan ^{-1}\left(\sqrt{\frac{(\omega / N)^{2}}{1-(\omega / N)^{2}}}\right) .
$$

${ }_{1191}$ The hydrostatic beam angle, given by Equation (57) for $\omega / N \ll 1$, is

$$
\varphi_{h}=\tan ^{-1}(\omega / N)
$$

In this numerical experiment, oscillatory flow over a Gaussian sill produces a strong signal of internal wave beams. The bathymetry is given by

$$
H=H_{0}-a_{b} \exp \left(-\frac{x^{2}}{2 L_{b}^{2}}\right)
$$

where the still water depth is $H_{0}=1000 \mathrm{~m}$, the sill amplitude is $a_{b}=20 \mathrm{~m}$ and $L_{b}=L / 100$. The domain is given by $x \in[-L / 2, L / 2]$, where length of the domain, $L$, is varied according to $L=4 H_{0} / \tan (\varphi)$ so that the beams reflect off the surface before encountering the open boundaries. Tidal forcing at the boundaries is given by

$$
u_{\mathrm{bc}}=u_{0} \sin (\omega t)
$$

where $u_{0}=0.01 \mathrm{~m} \mathrm{~s}^{-1}$. The parameters are chosen to ensure small values of $a_{b} / H_{0}$ and $u_{0} \omega / L_{b}$ (Llewellyn Smith and Young, 2002) to prevent nonlinear processes such as lee waves, higher harmonics, and associated mul- 
tiple beams. This numerical experiment is run with a constant stratification of $N=0.007 \mathrm{~s}^{-1}$ which corresponds to linearly-varying density with $\Delta \rho=5 \mathrm{~kg} \mathrm{~m}^{-3}$ and $\rho_{0}=1000 \mathrm{~kg} \mathrm{~m}^{-3}$. The forcing frequency $(\omega)$ of the oscillatory flow is varied to achieve different values of $\omega / N$. The model uses a high vertical resolution of 100 layers to resolve the higher internal wave modes which compose the beams. The model also uses sponge layers following Equation (53) of length $L_{s}=L / 10$ to absorb waves at the boundaries with a time scale of $\tau_{s}=100$. The model is run with $N_{x}=128$ grid points and a time step of $\Delta t=T_{\mathrm{bc}} / 500$ for a total time of 20 tidal periods $\left(T_{\mathrm{bc}}=2 \pi / \omega\right)$ so that the model has sufficient time to spin up (i.e. sufficient time for transients to decay and the beams to develop). Only nonhydrostatic simulations with $\omega / N>0.8$ require extensive spin-up time ${ }^{13}$ due to the nearly vertical energy propagation and slow horizontal velocity of (nonhydrostatic) high-mode waves. The model is run with a drag coefficient of $C_{d}=0.001$, a vertical viscosity of $\nu_{v}=1 \times 10^{-6} \mathrm{~m}^{2} \mathrm{~s}^{-1}$, and no horizontal viscosity.

Figure 15 shows the computational domain for the nonhydrostatic model with $\omega / N=0.8$. The figure shows a pseudo-color plot of the normalized perturbation velocity, $u^{\prime} / u_{0}=\left(u-u_{\mathrm{bc}}\right) / u_{0}$, at peak flood, $t=19.25 T_{\mathrm{bc}}$, which illustrates internal wave beams radiating away from the sill. Also note that, according to theory, the internal wave phase velocity vector is perpendicular to the beam angle. For reference, the theoretical nonhydrostatic (thick blue

\footnotetext{
${ }^{13}$ Approximately 10 tidal periods are needed for the higher modes to propagate over the entire domain and for the beams to develop.
} 


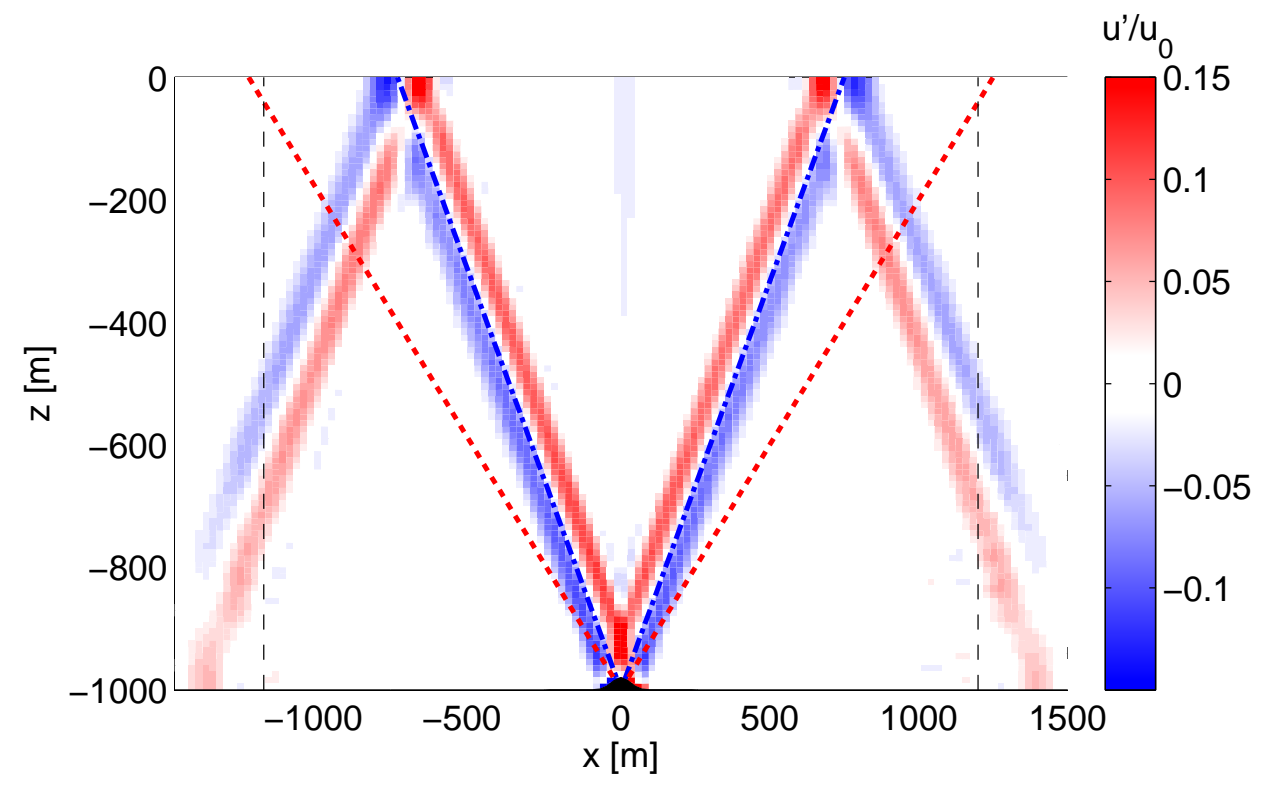

Figure 15: The setup for the internal wave beam numerical experiment. This figure shows the ridge and the normalized perturbation velocity $u^{\prime} / u_{0}=\left(u-u_{\mathrm{bc}}\right) / u_{0}$ associated with the internal wave beams at peak flood, $t=19.25 T_{\mathrm{bc}}$, for a forcing frequency of $\omega=0.8 \mathrm{~N}$ in the nonhydrostatic model. Also shown for reference are the theoretical nonhydrostatic (thick blue dashed-dot line) and hydrostatic (thick red dashed line) beam angles given by Equations (57) and (58), respectively. The locations of the sponge layers are shown as thin black dashed lines near the domain boundaries.

dashed-dot line) and hydrostatic (thick red dashed line) beam angles given by Equations (57) and (58), respectively.

Figure 16 shows the theoretical nonhydrostatic and hydrostatic internal wave beam angles, given by Equations (57) and (58), respectively, compared to the model as a function of $\omega / N$. To compute the beam angle of the model, we determine the location of the maximum of the (RMS) perturbation velocity averaged over the last ten tidal periods for each layer in a limited region $\left(x_{\min }=2 \Delta x, x_{\max }=0.75 L, z_{\min }=-0.80 H_{0}, z_{\max }=-0.15 H_{0}\right)$ surrounding the beam on the right-half of the sill. A linear least-squares fit 


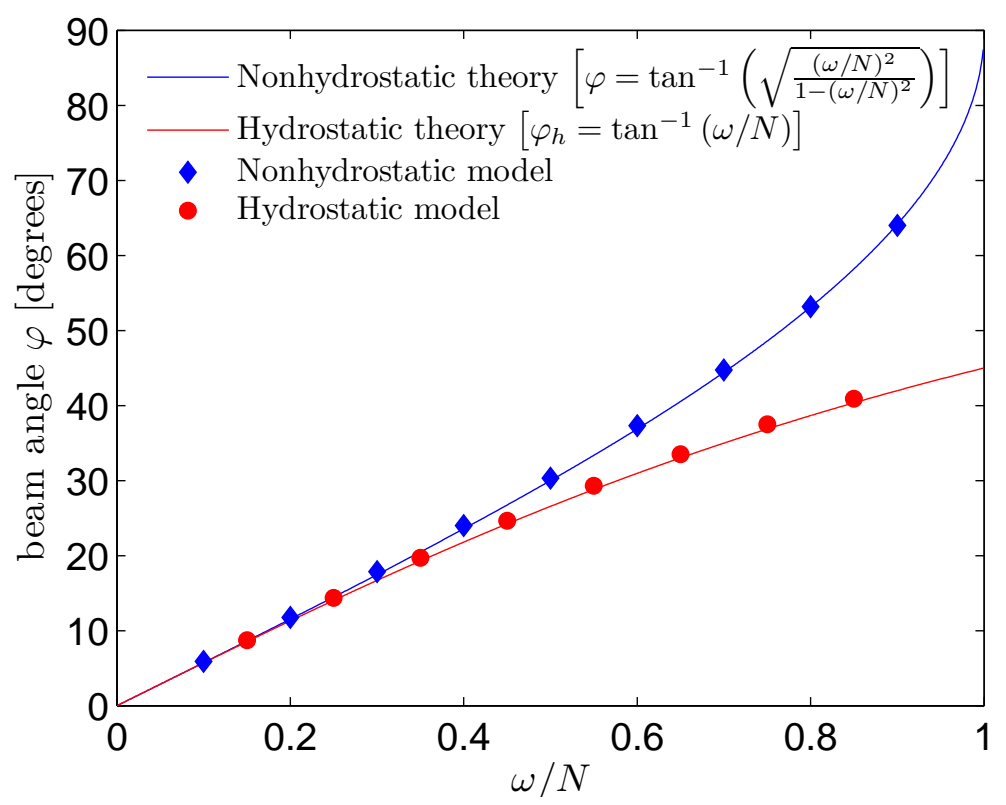

Figure 16: The internal wave beam angle $\varphi$ as a function of $\omega / N$. The theoretical curves for the internal wave beam angles are given in Equations (57) [solid blue line] and (58) [solid red line] for nonhydrostatic and hydrostatic internal waves, respectively. The beam angles determined from the model are given by the blue diamonds and red circles for the nonhydrostatic and hydrostatic models, respectively.

to the $(x-z)$ location of these maxima provides an estimate of the internal wave beam angle produced by the model.

As shown in Figure 16, the model correctly reproduces the hydrostatic and nonhydrostatic beam angles, which diverge when $\omega / N \gtrsim 0.3$. For $\omega / N \approx 1$, the nonhydrostatic beams become vertical, whereas the hydrostatic beams become nearly $45^{\circ}\left(\right.$ since $\left.\tan ^{-1}(1)=45^{\circ}\right)$. Ultimately, this test case demonstrates that the isopycnal model is capable of resolving internal wave behavior in smoothly stratified environments (i.e. not composed of a layered structure). 


\section{Conclusions}

This paper presents a nonhydrostatic ocean model with an isopycnal coordinate system. The governing model equations invoke a mild-slope approximation to the momentum equation and the divergence-free condition relating to the nonhydrostatic pressure. This approximation arises from scaling arguments and is valid for most oceanic flows of interest. The scaling analysis conducted here suggests that the nonhydrostatic pressure terms which are often neglected can be significantly larger than many metric terms that are often retained (such as those arising from viscosity). The mild-slope approximation reduces the elliptic equation for the nonhydrostatic pressure to a symmetric linear system. Ultimately, metric terms associated with nonhydrostatic pressure can be retained for flows with steep isopycnal slopes (where $z_{x^{*}}=\mathcal{O}(1)$ ) at the expense of increased computational cost of solving a nonsymmetric linear system with approximately double the bandwidth. Despite the approximations, the framework is in place to implement the nonhydrostatic pressure into existing isopycnal-coordinate ocean models. The method presented here is valid for a 2-D $(x-z)$ system, however the method is easily extended to 3-D by treating the north-south $(v)$ velocity in a similar manner to the east-west (u) velocity. Overall, the numerical method is second-order accurate in time and space and uses an implicit free-surface discretization that is similar to that in existing nonhydrostatic models using $z$ - or $\sigma$-coordinates. Although the use of a Lagrangian vertical coordinate and a nonhydrostatic projection method was reported to be mutually exclusive (Adcroft and Hallberg, 2006), 
we find that such a numerical formulation is possible at least to second-order accuracy. It appears that a solution to the conundrum of Adcroft and Hallberg (2006) may be possible in the context of numerical formulations of the Navier-Stokes equations in generalized vertical coordinates since it is not necessary to maintain absolute consistency between the layer-height continuity equation (7) and the divergence-free continuity equation (8). Instead, it is only necessary to obtain consistency between Equations (7) and (8) to a sufficient order of accuracy. In this case, we consider second-order accuracy to be sufficient because higher-order nonhydrostatic models are rare.

The numerical method is stable provided the time step satisfies the internal wave, wetting/drying, and horizontal advection and diffusion Courant numbers. The main limitation of this model, like all isopycnal-coordinate models, is the inability to represent unstable stratification and overturning motions which are effects typically associated with nonhydrostatic pressure. Overturning motions typically exist on small scales and, consequently, are often under-resolved in modeling applications. Parameterization of overturning motions in the context of isopycnal-coordinate models, especially in nonhydrostatic formulations such as the current model, is beyond the scope of this paper. However, this may provide interesting topics for future research - e.g. How can we parameterize internal wave breaking in isopycnal models? Can accounting for nonhydrostatic effects improve such a parameterization? Despite the limitation of the isopycnal-coordinate framework in that it does not allow for overturning motions, our model shows that addi- 
tion of the nonhydrostatic pressure to an isopycnal-coordinate ocean model allows for computation of nonhydrostatic internal waves which account for a substantial fraction of the energy spectrum spanning between the hydrostatic low-frequency motions and the high-frequency overturning motions.

We demonstrate that the model captures dispersive wave properties and simulates nonlinear internal solitary waves in idealized test cases and in a realistic oceanographic problem of internal solitary wave generation over a ridge. The model is capable of representing internal waves with a reduced number of vertical layers (ideally two). Additional layers may be necessary to capture the vertical variability of strongly nonhydrostatic flows. Ultimately, the model may provide an efficient formulation that is well-suited to simulations of nonhydrostatic internal gravity waves that are prohibitively expensive using $z$-level or $\sigma$-coordinate models.

\section{Acknowledgments}

The authors wish to thank Greg Ivey, Kyle Mandli, Maarten Buijsman, Jody Klymak, and three anonymous reviewers for their invaluable discussions and comments related to the model development and testing. S.V. gratefully acknowledges his support as a DOE Computational Science Graduate Fellow which is provided under grant number DE-FG02-97ER25308. S.V. and O.B.F. gratefully acknowledge the support of ONR as part of the YIP and PECASE awards under grants N00014-10-1-0521 and N00014-08-1-0904 (scientific officers Dr. C. Linwood Vincent, Dr. Terri Paluszkiewicz and Dr. 
Scott Harper).

\section{Appendix A. Nondimensionalization and coordinate transforma- tion}

The governing equations (2) are nondimensionalized according to

$$
\begin{gathered}
x^{\prime}=\frac{x}{L}, \quad y^{\prime}=\frac{y}{L}, \quad z^{\prime}=\frac{z}{H}, \quad t^{\prime}=\frac{t}{T}=\frac{c_{i} t}{L}, \\
u^{\prime}=\frac{u}{U}, \quad v^{\prime}=\frac{v}{U}, \quad w^{\prime}=\frac{w}{W}=\frac{w}{\epsilon U}, \quad \rho^{\prime}=\frac{\rho}{\rho_{0}}, \quad p_{h}^{\prime}=\frac{p_{h}}{\rho_{0} U c_{i}}, \quad q^{\prime}=\frac{q}{\epsilon^{2} U c_{i}},
\end{gathered}
$$

where $L$ is the horizontal length scale, $H$ is the vertical depth scale, $T$ is the time scale, $U$ is the horizontal velocity scale, $W$ is the vertical velocity scale, $c_{i}$ is the wave speed scale and $\epsilon=\frac{H}{L}$. The scale for the hydrostatic pressure is chosen to achieve a first-order balance between the unsteady term and the hydrostatic pressure gradient in the horizontal momentum equations. Likewise, the scale for the nonhydrostatic pressure is chosen to achieve a first-order balance between the inertia term and the nonhydrostatic pressure gradient in the vertical momentum equation. After applying the nondimensionalization, Equations (2) become 


$$
\begin{aligned}
& \frac{\partial u^{\prime}}{\partial t^{\prime}}+F \nabla \cdot\left(\mathbf{u}^{\prime} u^{\prime}\right)-\frac{F}{\mathrm{Ro}} v^{\prime}+\frac{F \epsilon}{\widetilde{\mathrm{Ro}_{0}}} w^{\prime}=-\frac{\partial p_{h}^{\prime}}{\partial x^{\prime}}-\epsilon^{2} \frac{\partial q^{\prime}}{\partial x^{\prime}}+\frac{F}{\operatorname{Re}_{H}} \nabla_{H}^{2} u^{\prime}+\frac{F}{\operatorname{Re}_{v}} \frac{\partial^{2} u^{\prime}}{\partial z^{\prime 2}} \\
& \frac{\partial v^{\prime}}{\partial t^{\prime}}+F \nabla \cdot\left(\mathbf{u}^{\prime} v^{\prime}\right)+\frac{F}{\mathrm{Ro}} u^{\prime} \quad=-\frac{\partial p_{h}^{\prime}}{\partial y^{\prime}}-\epsilon^{2} \frac{\partial q^{\prime}}{\partial y^{\prime}}+\frac{F}{\operatorname{Re}_{H}} \nabla_{H}^{2} v^{\prime}+\frac{F}{\operatorname{Re}_{v}} \frac{\partial^{2} v^{\prime}}{\partial z^{\prime 2}} \\
& \frac{\partial w^{\prime}}{\partial t^{\prime}}+F \nabla \cdot\left(\mathbf{u}^{\prime} w^{\prime}\right)-\frac{F}{\epsilon} \frac{1}{\widetilde{\mathrm{Ro}_{0}}} u^{\prime} \quad=\quad-\frac{\partial q^{\prime}}{\partial z^{\prime}}+\frac{F}{\operatorname{Re}_{H}} \nabla_{H}^{2} w^{\prime}+\frac{F}{\operatorname{Re}_{v}} \frac{\partial^{2} w^{\prime}}{\partial z^{\prime 2}}
\end{aligned}
$$

1321

where $F=\frac{u}{c_{i}}, \operatorname{Re}_{H}=\frac{U L}{\nu_{H}}, \operatorname{Re}_{v}=\frac{W H}{\nu_{v}}=\epsilon^{2} \frac{U L}{\nu_{v}}, \operatorname{Ro}=\frac{U}{f L}$, and $\widetilde{\operatorname{Ro}}=\frac{U}{\tilde{f} L}$ are the Froude, horizontal and vertical Reynolds, traditional and nontraditional Rossby numbers, respectively. In Equations (A.1), we have assumed a constant horizontal and vertical viscosity for convenience. These equations are subject to the nondimensional incompressibility constraint given by

$$
\frac{\partial u^{\prime}}{\partial x^{\prime}}+\frac{\partial v^{\prime}}{\partial y^{\prime}}+\frac{\partial w^{\prime}}{\partial z^{\prime}}=0
$$

${ }_{326}$ Applying the nondimensional equivalent of the transformation rules (5) results in the nondimensional equations in isopycnal coordinates 


$$
\begin{aligned}
& \frac{\partial u^{\prime}}{\partial t^{*^{\prime}}}+F\left(\mathbf{u}_{H^{*}}^{\prime} \cdot \nabla_{H^{*}} u^{\prime}\right)-\frac{F}{\mathrm{Ro}} v^{\prime}+\frac{F \epsilon}{\widetilde{\mathrm{Ro}}} w^{\prime}=-\frac{\partial M^{\prime}}{\partial x^{*^{\prime}}}-\epsilon^{2} \frac{\partial q^{\prime}}{\partial x^{*^{\prime}}}+\frac{F}{\operatorname{Re}_{H}} \nabla_{H^{*}}^{2} u^{\prime}+\frac{F}{\operatorname{Re}_{v}}\left(\frac{1}{z_{\rho^{\prime}}^{\prime}}\right)^{2} \frac{\partial^{2} u^{\prime}}{\partial \rho^{\prime 2}} \\
& +\epsilon^{2} \frac{z_{x^{*^{\prime}}}^{\prime}}{z_{\rho^{\prime}}^{\prime}} \frac{\partial q^{\prime}}{\partial \rho^{\prime}}+\frac{F}{\operatorname{Re}_{H}}\left[-2 \frac{z_{x^{*^{\prime}}}^{\prime}}{z_{\rho^{\prime}}^{\prime}} \frac{\partial^{2} u^{\prime}}{\partial x^{*^{\prime}} \partial \rho^{\prime}}-2 \frac{z_{y^{*^{\prime}}}^{\prime}}{z_{\rho^{\prime}}^{\prime}} \frac{\partial^{2} u^{\prime}}{\partial y^{*^{\prime}} \partial \rho^{\prime}}+\left(\frac{z_{x^{*^{\prime}}}^{\prime}}{z_{\rho^{\prime}}^{\prime}}\right)^{2} \frac{\partial^{2} u^{\prime}}{\partial \rho^{\prime 2}}+\left(\frac{z_{y^{*^{\prime}}}^{\prime}}{z_{\rho^{\prime}}^{\prime}}\right)^{2} \frac{\partial^{2} u^{\prime}}{\partial \rho^{\prime 2}}\right] \\
& \frac{\partial v^{\prime}}{\partial t^{*^{\prime}}}+F\left(\mathbf{u}_{H^{*}}^{\prime} \cdot \nabla_{H^{*}} v^{\prime}\right)+\frac{F}{\operatorname{Ro}} u^{\prime}=-\frac{\partial M^{\prime}}{\partial y^{*^{\prime}}}-\epsilon^{2} \frac{\partial q^{\prime}}{\partial y^{*^{\prime}}}+\frac{F}{\operatorname{Re}_{H}} \nabla_{H^{*}}^{2} v^{\prime}+\frac{F}{\operatorname{Re}_{v}}\left(\frac{1}{z_{\rho^{\prime}}^{\prime}}\right)^{2} \frac{\partial^{2} v^{\prime}}{\partial \rho^{\prime 2}} \\
& +\epsilon^{2} \frac{z_{y^{*^{\prime}}}^{\prime}}{z_{\rho^{\prime}}^{\prime}} \frac{\partial q^{\prime}}{\partial \rho^{\prime}}+\frac{F}{\operatorname{Re}_{H}}\left[-2 \frac{z_{x^{*^{\prime}}}^{\prime}}{z_{\rho^{\prime}}^{\prime}} \frac{\partial^{2} v^{\prime}}{\partial x^{*^{\prime}} \partial \rho^{\prime}}-2 \frac{z_{y^{*^{\prime}}}^{\prime}}{z_{\rho^{\prime}}^{\prime}} \frac{\partial^{2} v^{\prime}}{\partial y^{*^{\prime}} \partial \rho^{\prime}}+\left(\frac{z_{x^{*^{\prime}}}^{\prime}}{z_{\rho^{\prime}}^{\prime}}\right)^{2} \frac{\partial^{2} v^{\prime}}{\partial \rho^{\prime 2}}+\left(\frac{z_{y^{*^{\prime}}}^{\prime}}{z_{\rho^{\prime}}^{\prime}}\right)^{2} \frac{\partial^{2} v^{\prime}}{\partial \rho^{\prime 2}}\right] \\
& \frac{\partial w^{\prime}}{\partial t^{*^{\prime}}}+F\left(\mathbf{u}_{H^{*}}^{\prime} \cdot \nabla_{H^{*}} w^{\prime}\right)-\frac{F}{\epsilon} \frac{1}{\widetilde{\mathrm{Ro}_{0}}} u^{\prime}=-\frac{1}{z_{\rho^{\prime}}^{\prime}} \frac{\partial q^{\prime}}{\partial \rho^{\prime}}+\frac{F}{\operatorname{Re}_{H}} \nabla_{H^{*}}^{2} w^{\prime}+\frac{F}{\operatorname{Re}_{v}}\left(\frac{1}{z_{\rho^{\prime}}^{\prime}}\right)^{2} \frac{\partial^{2} w^{\prime}}{\partial \rho^{\prime 2}} \\
& +\frac{F}{\operatorname{Re}_{H}}\left[-2 \frac{z_{x^{*^{\prime}}}^{\prime}}{z_{\rho^{\prime}}^{\prime}} \frac{\partial^{2} w^{\prime}}{\partial x^{*^{\prime}} \partial \rho^{\prime}}-2 \frac{z_{y^{*^{\prime}}}^{\prime}}{z_{\rho^{\prime}}^{\prime}} \frac{\partial^{2} w^{\prime}}{\partial y^{*^{\prime}} \partial \rho^{\prime}}+\left(\frac{z_{x^{*^{\prime}}}^{\prime}}{z_{\rho^{\prime}}^{\prime}}\right)^{2} \frac{\partial^{2} w^{\prime}}{\partial \rho^{\prime 2}}+\left(\frac{z_{y^{*^{\prime}}}^{\prime}}{z_{\rho^{\prime}}^{\prime}}\right)^{2} \frac{\partial^{2} w^{\prime}}{\partial \rho^{\prime 2}}\right]
\end{aligned}
$$

where $M^{\prime}=p_{h}^{\prime}+\frac{1}{F} \rho^{\prime} z^{\prime}$ is the nondimensional Montgomery potential. Equations (A.3) include all of the metric terms associated with the coordinate transformation. Since $z_{\rho^{\prime}}^{\prime}=\mathcal{O}(1)$, terms containing $z_{\rho^{\prime}}^{\prime}$ cannot be neglected. However, many of the other metric terms can be safely neglected based on scaling arguments. For example, we can safely assume that the isopycnal slope terms $\left(z_{x^{*^{\prime}}}^{\prime}\right.$ and $\left.z_{y^{*^{\prime}}}^{\prime}\right)$ are small since they scale like $\frac{a}{L}$ where $a$ is the scale for the internal wave amplitude. We can rewrite this slope 
scaling as $\frac{a}{L}=\frac{a}{H} \frac{H}{L} \approx F \epsilon<\mathcal{O}(1)$, since we have assumed the first-order gravity-wave balance $\frac{a}{H} \approx \frac{u}{c_{i}}=F$. These small nondimensional parameters $\left(z_{x^{*^{\prime}}}^{\prime} \approx z_{y^{*^{\prime}}}^{\prime}<\mathcal{O}(1), \epsilon^{2}<\mathcal{O}(1), \frac{F}{\operatorname{Re}_{H}}<\mathcal{O}(1), \frac{F}{\operatorname{Re}_{v}}<\mathcal{O}(1)\right)$ are negligible (i.e. $\ll 1)$ when they appear together in products. Additional support in favor of neglecting these metric terms is given in Vitousek and Fringer (2011), who showed that, at coarse resolution, model truncation error from the $\mathcal{O}(1)$ terms in the governing equations can be larger than (asymptotically weak) physical terms such as the nonhydrostatic pressure. Thus, in the current model, we ignore the small metric terms in Equation (A.3) associated with the nonhydrostatic pressure and viscosity (the terms on the second line of each equation).

Equations (6) which appear in Section 2.2 are the dimensional version of Equations (A.3) which are subject to the mild-slope approximation and a horizontally and vertically varying viscosity.

Transformed to isopycnal coordinates, the nondimensional continuity equation (A.2) becomes

$$
\frac{\partial}{\partial x^{*^{\prime}}}\left(z_{\rho^{\prime}}^{\prime} u^{\prime}\right)+\frac{\partial}{\partial y^{*^{\prime}}}\left(z_{\rho^{\prime}}^{\prime} v^{\prime}\right)+\frac{\partial W^{\prime}}{\partial \rho^{\prime}}=0
$$

where $W^{\prime}=w^{\prime}-z_{x^{*^{\prime}}}^{\prime} u^{\prime}-z_{y^{*^{\prime}}}^{\prime} v^{\prime}$. Invoking the mild-slope approximation for the continuity equation reduces Equation (A.4) to

$$
\frac{\partial}{\partial x^{*^{\prime}}}\left(z_{\rho^{\prime}}^{\prime} u^{\prime}\right)+\frac{\partial}{\partial y^{*^{\prime}}}\left(z_{\rho^{\prime}}^{\prime} v^{\prime}\right)+\frac{\partial w^{\prime}}{\partial \rho^{\prime}}=0,
$$


since $w^{\prime}=W^{\prime}+\mathcal{O}\left(z_{x^{*^{\prime}}}^{\prime}, z_{y^{*^{\prime}}}^{\prime}\right)$. In contrast to Equations (A.3), Equation (A.4) does not contain products of small nondimensional parameters. We can still neglect the isopycnal slope terms in Equation (A.4) (thus leading to Equation (A.5)) because the primary purpose of this form of the continuity equation is the calculation of the nonhydrostatic pressure $q^{\prime}$ through an elliptic equation. Because the nonhydrostatic pressure gradient in the momentum equations (A.3) has a small nondimensional coefficient of $\epsilon^{2}$, the isopycnal slope term in Equation (A.4) can be safely neglected as if appearing in a product of small parameters. Further discussion of the mild-slope approximation for the continuity equation (A.4) and (A.5) in discrete form and the resulting symmetry of the linear system for the nonhydrostatic pressure are presented in Appendix D and Appendix E, respectively.

\section{Appendix B. Implicit free-surface equation}

To derive an implicit equation for the free surface we follow an approach similar to Casulli (1999) in which the hydrostatic provisional velocities, $\mathbf{u}_{i \pm 1 / 2}^{*}$, are substituted into the free-surface equation. In this approach, rather than use an equation for the free surface directly, we derive an equation for the free surface by summing over the layer-height continuity equation after substitution of the provisional velocities.

We can write the layer-height continuity equation (30) in matrix form as 


$$
\begin{aligned}
\frac{\mathbf{h}_{i}^{n+1}-\mathbf{h}_{i}^{n}}{\Delta t}= & -\frac{1}{2}(1+c) \frac{1}{\Delta x}\left(\mathbf{h}_{i+\frac{1}{2}}^{n} \circ \mathbf{u}_{i+\frac{1}{2}}^{*}-\mathbf{h}_{i-\frac{1}{2}}^{n} \circ \mathbf{u}_{i-\frac{1}{2}}^{*}\right) \\
& -\frac{1}{2}(1-2 c) \frac{1}{\Delta x}\left(\mathbf{h}_{i+\frac{1}{2}}^{n} \circ \mathbf{u}_{i+\frac{1}{2}}^{n}-\mathbf{h}_{i-\frac{1}{2}}^{n} \circ \mathbf{u}_{i-\frac{1}{2}}^{n}\right) \\
& -\frac{c}{2} \frac{1}{\Delta x} \quad\left(\mathbf{h}_{i+\frac{1}{2}}^{n} \circ \mathbf{u}_{i+\frac{1}{2}}^{n-1}-\mathbf{h}_{i-\frac{1}{2}}^{n} \circ \mathbf{u}_{i-\frac{1}{2}}^{n-1}\right),
\end{aligned}
$$

1373

1374

1375

1376

1377

$$
\begin{aligned}
\mathbf{h}_{i}^{n+1} & -g\left(\frac{1}{2}(1+c) \frac{\Delta t}{\Delta x}\right)^{2}\left\{\left(\mathbf{h}_{i+\frac{1}{2}}^{n} \circ \tilde{\mathbf{e}}_{i+\frac{1}{2}}\right) \eta_{i+1}^{n+1}-\left[\left(\mathbf{h}_{i+\frac{1}{2}}^{n} \circ \tilde{\mathbf{e}}_{i+\frac{1}{2}}\right)+\left(\mathbf{h}_{i-\frac{1}{2}}^{n} \circ \tilde{\mathbf{e}}_{i-\frac{1}{2}}\right)\right] \eta_{i}^{n+1}+\left(\mathbf{h}_{i-1 / 2}^{n} \circ \tilde{\mathbf{e}}_{i-\frac{1}{2}}\right) \eta_{i-1}^{n+1}\right\} \\
=\mathbf{h}_{i}^{n} & -\frac{1}{2}(1+c) \frac{\Delta t}{\Delta x}\left(\mathbf{h}_{i+\frac{1}{2}}^{n} \circ \tilde{\mathbf{T}}_{i+\frac{1}{2}}^{n}-\mathbf{h}_{i-\frac{1}{2}}^{n} \circ \tilde{\mathbf{T}}_{i-\frac{1}{2}}^{n}\right) \\
& -\frac{1}{2}(1-2 c) \frac{\Delta t}{\Delta x}\left(\mathbf{h}_{i+\frac{1}{2}}^{n} \circ \mathbf{u}_{i+\frac{1}{2}}^{n}-\mathbf{h}_{i-\frac{1}{2}}^{n} \circ \mathbf{u}_{i-\frac{1}{2}}^{n}\right) \\
& -\frac{c}{2} \frac{\Delta t}{\Delta x}\left(\mathbf{h}_{i+\frac{1}{2}}^{n} \circ \mathbf{u}_{i+\frac{1}{2}}^{n-1}-\mathbf{h}_{i-\frac{1}{2}}^{n} \circ \mathbf{u}_{i-\frac{1}{2}}^{n-1}\right) .
\end{aligned}
$$

1378 The free surface can be computed by summing over the layer heights as $1379 \eta_{i}=\sum_{k=1}^{N_{\text {layers }}} h_{i, k}-H_{i}=\mathbf{e}^{T} \mathbf{h}_{i}-H_{i}$. Therefore, we multiply Equation (B.2) 1380 by $\mathbf{e}^{T}$ and subtract the depth, $H_{i}$ from both sides to give 


$$
\begin{aligned}
& \eta_{i}^{n+1} \\
& -g\left(\frac{1}{2}(1+c) \frac{\Delta t}{\Delta x}\right)^{2} \mathbf{e}^{T}\left\{\left(\mathbf{h}_{i+\frac{1}{2}}^{n} \circ \tilde{\mathbf{e}}_{i+\frac{1}{2}}\right) \eta_{i+1}^{n+1}-\left[\left(\mathbf{h}_{i+\frac{1}{2}}^{n} \circ \tilde{\mathbf{e}}_{i+\frac{1}{2}}\right)+\left(\mathbf{h}_{i-\frac{1}{2}}^{n} \circ \tilde{\mathbf{e}}_{i-\frac{1}{2}}\right)\right] \eta_{i}^{n+1}+\left(\mathbf{h}_{i-1 / 2}^{n} \circ \tilde{\mathbf{e}}_{i-\frac{1}{2}}\right) \eta_{i-1}^{n+1}\right\} \\
& =\eta_{i}^{n}-\frac{1}{2}(1+c) \frac{\Delta t}{\Delta x}\left[\mathbf{e}^{T}\left(\mathbf{h}_{i+\frac{1}{2}}^{n} \circ \tilde{\mathbf{T}}_{i+\frac{1}{2}}^{n}\right)-\mathbf{e}^{T}\left(\mathbf{h}_{i-\frac{1}{2}}^{n} \circ \tilde{\mathbf{T}}_{i-\frac{1}{2}}^{n}\right)\right] \\
& -\frac{1}{2}(1-2 c) \frac{\Delta t}{\Delta x}\left[\mathbf{e}^{T}\left(\mathbf{h}_{i+\frac{1}{2}}^{n} \circ \mathbf{u}_{i+\frac{1}{2}}^{n}\right)-\mathbf{e}^{T}\left(\mathbf{h}_{i-\frac{1}{2}}^{n} \circ \mathbf{u}_{i-\frac{1}{2}}^{n}\right)\right] \\
& -\frac{c}{2} \frac{\Delta t}{\Delta x}\left[\mathbf{e}^{T}\left(\mathbf{h}_{i+\frac{1}{2}}^{n} \circ \mathbf{u}_{i+\frac{1}{2}}^{n-1}\right)-\mathbf{e}^{T}\left(\mathbf{h}_{i-\frac{1}{2}}^{n} \circ \mathbf{u}_{i-\frac{1}{2}}^{n-1}\right)\right] .
\end{aligned}
$$

${ }_{1381}$ Thus Equation (B.3) can be simplified further to

$$
-\left(\alpha_{1}\right)_{i-\frac{1}{2}}^{n} \eta_{i-1}^{n+1}-\left[1+\left(\alpha_{1}\right)_{i-\frac{1}{2}}^{n}+\left(\alpha_{1}\right)_{i+\frac{1}{2}}^{n}\right] \eta_{i}^{n+1}-\left(\alpha_{1}\right)_{i+\frac{1}{2}}^{n} \eta_{i+1}^{n+1}=R_{i}^{n}
$$

1382 1383

$$
\begin{aligned}
R_{i}^{n}=\eta_{i}^{n} & -\frac{1}{2}(1+c) \frac{\Delta t}{\Delta x}\left[\mathbf{e}^{T}\left(\mathbf{h}_{i+\frac{1}{2}}^{n} \circ \tilde{\mathbf{T}}_{i+\frac{1}{2}}^{n}\right)-\mathbf{e}^{T}\left(\mathbf{h}_{i-\frac{1}{2}}^{n} \circ \tilde{\mathbf{T}}_{i-\frac{1}{2}}^{n}\right)\right] \\
& -\frac{1}{2}(1-2 c) \frac{\Delta t}{\Delta x}\left[\mathbf{e}^{T}\left(\mathbf{h}_{i+\frac{1}{2}}^{n} \circ \mathbf{u}_{i+\frac{1}{2}}^{n}\right)-\mathbf{e}^{T}\left(\mathbf{h}_{i-\frac{1}{2}}^{n} \circ \mathbf{u}_{i-\frac{1}{2}}^{n}\right)\right] \\
& -\frac{c}{2} \frac{\Delta t}{\Delta x}\left[\mathbf{e}^{T}\left(\mathbf{h}_{i+\frac{1}{2}}^{n} \circ \mathbf{u}_{i+\frac{1}{2}}^{n-1}\right)-\mathbf{e}^{T}\left(\mathbf{h}_{i-\frac{1}{2}}^{n} \circ \mathbf{u}_{i-\frac{1}{2}}^{n-1}\right)\right] .
\end{aligned}
$$




\section{Appendix C. MPDATA to compute flux-face heights}

The MPDATA method to compute layer heights for volume fluxes involves first solving the first-order upwind or donor-cell approach using

$$
h_{i, k}^{*}=h_{i, k}^{n}-\left[F\left(h_{i, k}^{n}, h_{i+1, k}^{n}, U_{i+\frac{1}{2}, k}^{(n+1)_{\operatorname{Im}^{*}}}\right)-F\left(h_{i-1, k}^{n}, h_{i, k}^{n}, U_{i-\frac{1}{2}, k}^{(n+1)_{\mathrm{Im}^{*}}}\right)\right],
$$

where the fluxes are

$$
F\left(h_{i, k}^{n}, h_{i+1, k}^{n}, U_{i+\frac{1}{2}, k}^{(n+1)_{\mathrm{Im} *}}\right)=U_{i+\frac{1}{2}, k}^{+} h_{i, k}^{n}+U_{i+\frac{1}{2}, k}^{-} h_{i+1, k}^{n}
$$

where

$$
U_{i+\frac{1}{2}, k}^{ \pm}=\frac{1}{2}\left(U_{i+\frac{1}{2}, k}^{(n+1)_{\mathrm{Im}^{*}}} \pm\left|U_{i+\frac{1}{2}, k}^{(n+1)_{\mathrm{Im}^{*}}}\right|\right)
$$

and

$$
U_{i+\frac{1}{2}, k}^{(n+1)_{\mathrm{Im}^{*}}}=\frac{u_{i+\frac{1}{2}, k}^{(n+1)_{\mathrm{Im}^{*}}} \Delta t}{\Delta x},
$$

is the advective Courant number. We can correct the numerical diffusion associated with this step by applying an anti-diffusive correction step using the MPDATA approach of Smolarkiewicz and Margolin (1998). This correction is given by

$$
h_{i, k}^{n+1}=h_{i, k}^{*}-\left[F\left(h_{i, k}^{*}, h_{i+1, k}^{*}, \tilde{U}_{i+\frac{1}{2}, k}^{(n+1)_{\operatorname{Im}^{*}}}\right)-F\left(h_{i-1, k}^{*}, h_{i, k}^{*}, \tilde{U}_{i-\frac{1}{2}, k}^{(n+1)_{\operatorname{Im}}}\right)\right]
$$


1395

1396

1397

where

$$
\tilde{U}_{i+\frac{1}{2}, k}^{(n+1)_{\mathrm{Im}^{*}}}=\left(\left|U_{i+\frac{1}{2}, k}^{(n+1)_{\mathrm{Im}^{*}}}\right|-\left(U_{i+\frac{1}{2}, k}^{(n+1)_{\mathrm{Im}^{*}}}\right)^{2}\right) A_{i+\frac{1}{2}, k}^{*},
$$

and

$$
A_{i+\frac{1}{2}, k}^{*}=\left\{\begin{array}{cl}
\frac{h_{i+1, k}^{*}-h_{i, k}^{*}}{h_{i+1, k}^{*}+h_{i, k}^{*}} & \text { if } h_{i+1, k}^{*}+h_{i, k}^{*} \neq 0 \\
0 & \text { otherwise }
\end{array} .\right.
$$

Using MPDATA ensures second-order accuracy in time and space while also ensuring positive layer heights.

\section{Appendix D. Divergence-free continuity equation}

The continuity equation in continuous isopycnal coordinates in $2-\mathrm{D}$ is given by

$$
\frac{\partial}{\partial x}\left(z_{\rho} u\right)+\frac{\partial}{\partial \rho}\left(w-z_{x^{*}} u\right)=0
$$

Discretizing this equation results in

$$
\begin{aligned}
& \frac{1}{\Delta x}\left[\left(\frac{z_{i+\frac{1}{2}, k+\frac{1}{2}}^{n+1}-z_{i+\frac{1}{2}, k-\frac{1}{2}}^{n+1}}{\Delta \rho_{k}}\right) u_{i+\frac{1}{2}, k}^{n+1}-\left(\frac{z_{i-\frac{1}{2}, k+\frac{1}{2}}^{n+1}-z_{i-\frac{1}{2}, k-\frac{1}{2}}^{n+1}}{\Delta \rho_{k}}\right) u_{i-\frac{1}{2}, k}^{n+1}\right] \\
& +\frac{w_{i, k+\frac{1}{2}}^{n+1}-w_{i, k-\frac{1}{2}}^{n+1}}{\Delta \rho_{k}}-\frac{\left(z_{x^{*}}\right)_{i, k+\frac{1}{2}}^{n+1} u_{i, k+\frac{1}{2}}^{n+1}-\left(z_{x^{*}}\right)_{i, k-\frac{1}{2}}^{n+1} u_{i, k-\frac{1}{2}}^{n+1}}{\Delta \rho_{k}}=0 .
\end{aligned}
$$

Factoring the $1 / \Delta \rho_{k}$ from this equation and writing $z_{k+\frac{1}{2}}-z_{k-\frac{1}{2}}=z_{\text {bottom }}-$ $z_{\text {top }}=-\left(z_{\text {top }}-z_{\text {bottom }}\right)=-h_{k}$, gives the full, divergence-free continuity equation to be 


$$
\begin{aligned}
& \frac{1}{\Delta x}\left(h_{i+\frac{1}{2}, k}^{n+1} u_{i+\frac{1}{2}, k}^{n+1}-h_{i-\frac{1}{2}, k}^{n+1} u_{i-\frac{1}{2}, k}^{n+1}\right)+w_{i, k-\frac{1}{2}}^{n+1}-w_{i, k+\frac{1}{2}}^{n+1} \\
& -\beta\left(\left(z_{x^{*}}\right)_{i, k-\frac{1}{2}}^{n+1} u_{i, k-\frac{1}{2}}^{n+1}-\left(z_{x^{*}}\right)_{i, k+\frac{1}{2}}^{n+1} u_{i, k+\frac{1}{2}}^{n+1}\right)=0 .
\end{aligned}
$$

1406 The metric term $z_{x^{*}}$ is calculated as $z_{x^{*}}=\frac{\partial z}{\partial x^{*}}=\frac{z_{i+\frac{1}{2}}-z_{i-\frac{1}{2}}}{\Delta x}$ and $\beta=1$ or 0

depending on whether the metric terms are included or not, respectively. We generally use the simplified continuity equation, $\beta=0$. However, the full continuity equation is required when the isopycnal slopes are significant.

\section{Appendix E. Nonhydrostatic elliptic equation}

The full continuity equation in 2-D is given by Equation (D.2). In this equation, the velocity $u_{i, k \pm \frac{1}{2}}^{n+1}$ appearing as a product with the slope term, $z_{x^{*}}$, is undefined at these locations. Hence, we obtain its value using the following interpolation

$$
u_{i, k \pm \frac{1}{2}}^{n+1}=\frac{1}{4}\left(u_{i+\frac{1}{2}, k}^{n+1}+u_{i-\frac{1}{2}, k}^{n+1}+u_{i+\frac{1}{2}, k \pm 1}^{n+1}+u_{i-\frac{1}{2}, k \pm 1}^{n+1}\right) .
$$

Substituting Equation (E.1) into the continuity equation (D.2) gives 


$$
\begin{aligned}
& \frac{1}{\Delta x}\left(h_{i+\frac{1}{2}, k}^{n+1} u_{i+\frac{1}{2}, k}^{n+1}-h_{i-\frac{1}{2}, k}^{n+1} u_{i-\frac{1}{2}, k}^{n+1}\right)+w_{i, k-\frac{1}{2}}^{n+1}-w_{i, k+\frac{1}{2}}^{n+1} \\
& -\frac{\beta}{4}\left(z_{x^{*}}\right)_{i, k-\frac{1}{2}}^{n+1}\left(u_{i+\frac{1}{2}, k}^{n+1}+u_{i-\frac{1}{2}, k}^{n+1}+u_{i+\frac{1}{2}, k-1}^{n+1}+u_{i-\frac{1}{2}, k-1}^{n+1}\right) \\
& +\frac{\beta}{4}\left(z_{x^{*}}\right)_{i, k+\frac{1}{2}}^{n+1}\left(u_{i+\frac{1}{2}, k}^{n+1}+u_{i-\frac{1}{2}, k}^{n+1}+u_{i+\frac{1}{2}, k+1}^{n+1}+u_{i-\frac{1}{2}, k+1}^{n+1}\right)=0 .
\end{aligned}
$$

1416 The nonhydrostatic elliptic equation, derived by substituting Equations (11) 1417 into Equation (E.2), is then given by

$$
\begin{aligned}
& \frac{\Delta x}{h_{i, k-\frac{1}{2}}^{n+1}}\left(q_{c}\right)_{i, k-1}^{n+\frac{1}{2}}+\left[\frac{h_{i-\frac{1}{2}, k}^{n+1}}{\Delta x}-\frac{\beta}{4}\left(\left(z_{x^{*}}\right)_{i, k+\frac{1}{2}}^{n+1}-\left(z_{x^{*}}\right)_{i, k-\frac{1}{2}}^{n+1}\right)\right]\left(q_{c}\right)_{i-1, k}^{n+\frac{1}{2}} \\
& -\left(\frac{h_{i+\frac{1}{2}, k}^{n+1}}{\Delta x}+\frac{h_{i-\frac{1}{2}, k}^{n+1}}{\Delta x}+\frac{\Delta x}{h_{i, k+\frac{1}{2}}^{n+1}}+\frac{\Delta x}{h_{i, k-\frac{1}{2}}^{n+1}}\right)\left(q_{c}\right)_{i, k}^{n+\frac{1}{2}} \\
& \frac{\Delta x}{h_{i, k+\frac{1}{2}}^{n+1}}\left(q_{c}\right)_{i, k+1}^{n+\frac{1}{2}}+\left[\frac{h_{i+\frac{1}{2}, k}^{n+1}}{\Delta x}+\frac{\beta}{4}\left(\left(z_{x^{*}}\right)_{i, k+\frac{1}{2}}^{n+1}-\left(z_{x^{*}}\right)_{i, k-\frac{1}{2}}^{n+1}\right)\right]\left(q_{c}\right)_{i+1, k}^{n+\frac{1}{2}} \\
& -\frac{\beta}{4}\left(z_{x^{*}}\right)_{i, k-\frac{1}{2}}^{n+1}\left(\left(q_{c}\right)_{i+1, k-1}^{n+\frac{1}{2}}-\left(q_{c}\right)_{i-1, k-1}^{n+\frac{1}{2}}\right) \\
& +\frac{\beta}{4}\left(z_{x^{*}}\right)_{i, k+\frac{1}{2}}^{n+1}\left(\left(q_{c}\right)_{i+1, k+1}^{n+\frac{1}{2}}-\left(q_{c}\right)_{i-1, k+1}^{n+\frac{1}{2}}\right)=R_{i, k}^{*},
\end{aligned}
$$

where 


$$
\begin{aligned}
R_{i, k}^{*}= & \frac{\Delta x}{\Delta t}\left[\frac{1}{\Delta x}\left(h_{i+\frac{1}{2}, k}^{n+1} u_{i+\frac{1}{2}, k}^{*}-h_{i-\frac{1}{2}, k}^{n+1} u_{i-\frac{1}{2}, k}^{*}\right)+w_{i, k-\frac{1}{2}}^{*}-w_{i, k+\frac{1}{2}}^{*}\right] \\
& -\frac{\beta}{4} \frac{\Delta x}{\Delta t}\left(z_{x^{*}}\right)_{i, k-\frac{1}{2}}^{n+1}\left(u_{i+\frac{1}{2}, k}^{*}+u_{i-\frac{1}{2}, k}^{*}+u_{i+\frac{1}{2}, k-1}^{*}+u_{i-\frac{1}{2}, k-1}^{*}\right) \\
& +\frac{\beta}{4} \frac{\Delta x}{\Delta t}\left(z_{x^{*}}\right)_{i, k+\frac{1}{2}}^{n+1}\left(u_{i+\frac{1}{2}, k}^{*}+u_{i-\frac{1}{2}, k}^{*}+u_{i+\frac{1}{2}, k+1}^{*}+u_{i-\frac{1}{2}, k+1}^{*}\right) .
\end{aligned}
$$

Equation (E.3) constitutes a non-symmetric 9-diagonal matrix in 2-D. We note that Equation (E.3) represents the next order asymptotic approximation to the mild-slope elliptic equation and including the nonhydrostatic metric terms in the momentum equations (A.3) in the correction step (11) will result in the full elliptic equation. Equation (E.3) can be simplified to a symmetric 5-diagonal matrix in 2-D by neglecting the terms that appear in products with the isopycnal slope terms, i.e. $\beta=0$. This approximation is justified by scaling arguments of the isopycnal slope term and the fact that the nonhydrostatic terms scale as $\epsilon^{2}$ in the momentum equation (A.3) which leads to a product of small nondimensional parameters. If these terms were included, it would require extremely high grid resolution to ensure that the magnitude of the additional terms is larger than the truncation error associated with the $\mathcal{O}(1)$ terms (Vitousek and Fringer, 2011). When these approximations are made $\beta=0$ and Equation (E.3) becomes Equation (34). This approximation is very similar to the approximation made in Berntsen and Furnes (2005) which represents a mild-slope approximation to the nonhydrostatic elliptic equation in $\sigma$-coordinates. As shown in Keilegavlen and Berntsen 
(2009), simulations using the simplified elliptic equation of Berntsen and Furnes (2005) produce results that are nearly identical to simulations using the full elliptic equation in $\sigma$-coordinates.

\section{Appendix F. Internal wave generation in the South China Sea: Procedure to determine initial layer heights}

This appendix describes the procedure to determine the layer heights and densities in the 2-layer model by matching the mode-1 wave speed and solitary wave speed with the theoretical wave speed based on the stratification given in Equation (51).

Solitary wave behavior is approximately represented by the KdV equation

$$
\frac{\partial \xi}{\partial t}+c_{1} \frac{\partial \xi}{\partial x}+\delta \xi \frac{\partial \xi}{\partial x}+\epsilon \frac{\partial^{3} \xi}{\partial x^{3}}=0
$$

where $\xi$ is either the interface displacement or mode- 1 amplitude. The solitary wave solution to Equation (F.1) is given by

$$
\xi=a \operatorname{sech}^{2}\left(\frac{x-c t}{L_{0}}\right),
$$

where

$$
c=c_{1}+\frac{\delta a}{3},
$$

is the solitary wave speed with $c_{1}$ the linear, mode- 1 wave speed determined 
1450

from a modal analysis, and

$$
L_{0}=\sqrt{\frac{12}{a} \frac{\epsilon}{\delta}}
$$

1451 1452

1453

and

$$
\epsilon=\frac{1}{2} \frac{\int_{-H_{0}}^{0} \phi^{2} d z}{\int_{-H_{0}}^{0}\left(\frac{d \phi}{d z}\right)^{2} d z},
$$

1454

1455

1456

1457

1458

1459

1460

1461

1462

1463

1464

1465

is the solitary wave length (Liu, 1988; Helfrich and Melville, 2006). Following Liu (1988), the coefficients in Equation (F.1) are given by

$$
\delta=\frac{3}{2} c_{1} \frac{\int_{-H_{0}}^{0}\left(\frac{d \phi}{d z}\right)^{3} d z}{\int_{-H_{0}}^{0}\left(\frac{d \phi}{d z}\right)^{2} d z}
$$

where $\phi=\phi(z)$ is the mode-1 eigenfunction determined from a modal analysis.

Equations (F.5) and (F.6) are used to compute the values of $\delta$ and $\epsilon$ from the stratification in Equation (51) using an eigenfunction analysis (Fringer and Street, 2003) with 1000 equispaced vertical layers. These values along with the mode- 1 wave speed, $c_{1}$, are indicated in Table F.3, and are referred to as "Normal mode theory" values. The still-water interface depths in the 10-layer isopycnal model are arbitrarily set to $z=$ - $[0,50,100,250,460,750,1000,1500,2000,2500,3000] \mathrm{m}$ with density values computed at the vertical center of each layer using equation (51). Using these values of the layer heights and densities to compute the first-mode eigenfunction gives $c_{1}, \delta$, and $\epsilon$ that match the theoretical values quite well 
as shown in Table F.3. For the two-layer isopycnal model, the layer depths and densities are set to match the theoretical values for $c_{1}, \delta$, and $\epsilon$ from Helfrich and Melville (2006) that are given by

$$
\begin{aligned}
c_{1} & =\sqrt{2 \frac{\rho_{2}-\rho_{1}}{\rho_{1}+\rho_{2}} \frac{h_{1} h_{2}}{h_{1}+h_{2}}}, \\
\delta & =\frac{3}{2} c_{1} \frac{h_{1}-h_{2}}{h_{1} h_{2}}, \\
\epsilon & =\frac{1}{6} c_{1} h_{1} h_{2},
\end{aligned}
$$

respectively. For given values of $\delta$ and $\epsilon$, Equations (F.8) and (F.9) can be used to derive expressions for $h_{1}$ (since specifying $h_{1}$ also sets the value of $h_{2}$, i.e. $\left.h_{2}=H_{0}-h_{1}\right)$ which are given by

$$
\begin{aligned}
h_{1} & =\frac{1}{2} H_{0}-\frac{3}{2} \frac{c_{1}}{\delta} & \pm \frac{1}{2} \sqrt{H_{0}^{2}-9\left(\frac{c_{1}}{\delta}\right)^{2}}, \\
h_{1} & =\frac{1}{2} H_{0} & \pm \frac{1}{2} \sqrt{H_{0}^{2}-24 \frac{\epsilon}{c_{1}}} .
\end{aligned}
$$

Based on the form of Equations (F.10) and (F.11), it is, in general, not possible find a value of $h_{1}$ to match both the $\delta$ and $\epsilon$ coefficients with only two layers. For example, using values of $c_{1}=2.856 \mathrm{~m} \mathrm{~s}^{-1}, \delta=-0.0076 \mathrm{~s}^{-1}$, and $\epsilon=2.895 \times 10^{5} \mathrm{~m}^{3} \mathrm{~s}^{-1}$ from the 10-layer model, Equation (F.10) gives $h_{1}=3662 \mathrm{~m}$ or $h_{1}=460 \mathrm{~m}$ and Equation (F.11) gives $h_{1}=3191 \mathrm{~m}$ or $h_{1}=-191 \mathrm{~m}$. Clearly, since $H_{0}=3000 \mathrm{~m}$, the only possible choice for the 
upper layer thickness, $h_{1}$, is $h_{1}=460 \mathrm{~m}$ (and accordingly $h_{2}=H_{0}-H_{1}=$ $2540 \mathrm{~m}$ ). This choice allows the coefficient of nonlinearity, $\delta$, to match in the 2-layer and 10-layer models. Here, we match the coefficient of nonlinearity, $\delta$, in the 2- and 10-layer models to best match the solitary wave speed, $c$, which is dependent on $\delta$ as shown in Equation (F.3). We note that there are a number of viable alternative methods to compute $h_{1}$. For example, it is possible to select $h_{1}$ to match the ratio of $\frac{\epsilon}{\delta}$ and thereby match the solitary wave width, $L_{0}$, which depends on this ratio as shown in Equation (F.4). It may be also possible to choose $h_{1}$ to match quantities of interest such as the energy flux according to linear theory. However, development of an ideal method to choose $h_{1}$ is beyond the scope of this paper. With values of $h_{1}$ and $h_{2}$ known, the density difference between the layers is specified by matching the linear 2-layer wave speed from Equation (F.7). In the 2layer model, the density of the upper layer, $\rho_{1}=1028 \mathrm{~kg} \mathrm{~m}^{-3}$, is set using Equation (51) at the center of the layer. Next, the density of the lower layer, $\rho_{2}=1030.1967 \mathrm{~kg} \mathrm{~m}^{-3}$, is calculated to match the mode- 1 wave speed of the 10-layer model. Compilation of the KdV parameters based on normal-mode theory and the 2- and 10-layer models is given in Table F.3. Finally, we note that while the layer configurations should produce similar behavior for weakly nonlinear, mode-1 solitary wave behavior, nonlinear and higher-mode effects will produce results in the isopycnal model simulations that differ from the normal-mode theory. 
Table F.3: Matching parameters for the 2- and 10-layer models vs. normal model theory.

\begin{tabular}{lccc}
\hline & $c_{1}\left[\mathrm{~m} \mathrm{~s}^{-1}\right]$ & $\delta\left[\mathrm{s}^{-1}\right]$ & $\epsilon\left[\mathrm{m}^{3} \mathrm{~s}^{-1}\right]$ \\
\hline Normal mode theory & 2.866 & -0.0076 & $2.94 \times 10^{5}$ \\
10-layer model & 2.856 & -0.0076 & $2.89 \times 10^{5}$ \\
2-layer model & 2.856 & -0.0076 & $5.56 \times 10^{5}$ \\
\hline
\end{tabular}




\section{References}

Adcroft, A., Hallberg, R., 2006. On methods for solving the oceanic equations of motion in generalized vertical coordinates. Ocean Modelling 11, 224233.

Armfield, S., Street, R., 2002. An analysis and comparison of the time accuracy of fractional-step methods for the Navier-Stokes equations on staggered grids. International journal for numerical methods in fluids 38, 255-282.

Armfield, S., Street, R., 2003. The pressure accuracy of fractional-step methods for the Navier-Stokes equations on staggered grids. ANZIAM Journal 44, C20-C39.

Armfield, S.W., Street, R.L., 2000. Fractional step methods for the NavierStokes equations on non-staggered grids. ANZIAM J. 42(E), C134-C156.

Barad, M., Fringer, O., 2010. Simulations of shear instabilities in interfacial gravity waves. Journal of Fluid Mechanics 644, 61-95.

Berntsen, J., Furnes, G., 2005. Internal pressure errors in $\sigma$-coordinate ocean models-sensitivity of the growth of the flow to the time stepping method and possible non-hydrostatic effects. Continental shelf research 25, 829848.

Bleck, R., 2002. An oceanic general circulation model framed in hybrid isopycnic-Cartesian coordinates. Ocean Modelling 4, 55-88. 
Bleck, R., Boudra, D., 1981. Initial testing of a numerical ocean circulation model using a hybrid (quasi-isopycnic) vertical coordinate. Journal of Physical Oceanography 11, 755-770.

Bleck, R., Rooth, C., Hu, D., Smith, L., 1992. Salinity-driven thermocline transients in a wind-and thermohaline-forced isopycnic coordinate model of the North Atlantic. Journal of Physical Oceanography 22, 1486-1486.

Bleck, R., Smith, L.T., 1990. A wind-driven isopycnic coordinate model of the North and Equatorial Atlantic Ocean: 1. Model development and supporting experiments. Journal of Geophysical Research: Oceans 95, $3273-3285$.

Bogucki, D., Garrett, C., 1993. A simple model for the shear induced decay of an internal solitary wave. J. Phys. Oceanogr. 23, 1767-1776.

Brandt, P., Rubino, A., Alpers, W., Backhaus, J.O., 1997. Internal waves in the Strait of Messina studied by a numerical model and synthetic aperture radar images from the ERS 1/2 satellites. Journal of Physical Oceanography 27, 648-663.

Briggs, W.L., Henson, V.E., McCormick, S.F., 2000. A multigrid tutorial: Second edition. Society for Industrial and Applied Mathematics, Philadelphia, PA, USA.

Buijsman, M.C., Kanarska, Y., McWilliams, J.C., 2010. On the generation 
and evolution of nonlinear internal waves in the South China Sea. Journal of Geophysical Research: Oceans 115, n/a-n/a.

Castro-Díaz, M., Fernández-Nieto, E., González-vida, J., Parés, C., 2011. Numerical treatment of the loss of hyperbolicity ofthetwo-layer shallowwater system. Journal of Scientific Computing 48, 16-40.

Casulli, V., 1990. Semi-implicit finite difference methods for the twodimensional shallow water equations. J. Comp. Phys. 86, 56-74.

Casulli, V., 1997. Numerical simulation of three-dimensional free surface flow in isopycnal co-ordinates. International Journal for Numerical Methods in Fluids 25, 645-658.

Casulli, V., 1999. A semi-implicit finite difference method for nonhydrostatic, free-surface flows. Int. J. Numer. Methods Fluids 30, 425-440.

Casulli, V., Cattani, E., 1994. Stability, accuracy and efficiency of a semiimplicit method for three-dimensional shallow water flow. Computers and Mathematics with Applications 27, 99 - 112.

Chassignet, E., Arango, H., Dietrich, D., Ezer, T., Ghil, M., Haidvogel, D., Ma, C., Mehra, A., Paiva, A., Sirkes, Z., 2000. DAMEE-NAB: The base experiments. Dynamics of Atmospheres and Oceans 32, 155-183.

Chassignet, E.P., 2011. Isopycnic and hybrid ocean modeling in the context of GODAE, in: Schiller, A., Brassington, G.B. (Eds.), Operational Oceanography in the 21st Century. Springer Netherlands, pp. 263-293. 
Choi, W., Barros, R., Jo, T.C., 2009. A regularized model for strongly nonlinear internal solitary waves. Journal of Fluid Mechanics 629, 73.

Choi, W., Camassa, R., 1999. Fully nonlinear internal waves in a two-fluid system. Journal of Fluid Mechanics 396, 1-36.

Chumakova, L., Menzaque, F.E., Milewski, P.A., Rosales, R.R., Tabak, E.G., Turner, C.V., 2009. Shear instability for stratified hydrostatic flows. Communications on Pure and Applied Mathematics 62, 183-197.

Crank, J., Nicolson, P., 1947. A practical method for numerical evaluation of solutions of partial differential equations of the heat-conduction type. Mathematical Proceedings of the Cambridge Philosophical Society 43, 5067.

de la Fuente, A., Shimizu, K., Imberger, J., Niño, Y., 2008. The evolution of internal waves in a rotating, stratified, circular basin and the influence of weakly nonlinear and nonhydrostatic accelerations. Limnology and Oceanography 53, pp. 2738-2748.

de Szoeke, R.A., 2000. Equations of motion using thermodynamic coordinates. Journal of Physical Oceanography 30, 2814-2829.

Durran, D., 1991. The third-order Adams-Bashforth method: An attractive alternative to leapfrog time differencing. Monthly weather review 119, $702-720$. 
Durran, D.R., Blossey, P.N., 2012. Implicit-explicit multistep methods for fast-wave-slow-wave problems. Monthly Weather Review 140, 1307-1325.

Fiebig-Wittmaack, M., Börsch-Supan, W., Bischoff-Gauss, I., Astudillo, O., 2011. Tridiagonal preconditioning for Poisson-like difference equations with flat grids: Application to incompressible atmospheric flow. Journal of Computational and Applied Mathematics 236, 1435 - 1441.

Ford, R., Pain, C., Piggott, M., Goddard, A., de Oliveira, C., Umpleby, A., 2004a. A nonhydrostatic finite-element model for three-dimensional stratified oceanic flows. Part I: Model formulation. Monthly Weather Review $132,2816-2831$.

Ford, R., Pain, C., Piggott, M., Goddard, A., de Oliveira, C., Umpleby, A., 2004b. A nonhydrostatic finite-element model for three-dimensional stratified oceanic flows. Part II: Model validation. Monthly weather review $132,2832-2844$.

Fringer, O.B., Armfield, S.W., Street, R.L., 2005. Reducing numerical diffusion in interfacial gravity wave simulations. International Journal for Numerical Methods in Fluids 49, 301-329.

Fringer, O.B., Gerritsen, M., Street, R.L., 2006. An unstructured-grid, finitevolume, nonhydrostatic, parallel coastal ocean simulator. Ocean Modelling $14,139-173$. 
Fringer, O.B., Street, R.L., 2003. The dynamics of breaking progressive interfacial waves. J. Fluid Mech. 494, 319-353.

Gerkema, T., Zimmerman, J., Maas, L., Van Haren, H., 2008. Geophysical and astrophysical fluid dynamics beyond the traditional approximation. Reviews of Geophysics 46.

Griffies, S., Böning, C., Bryan, F., Chassignet, E., Gerdes, R., Hasumi, H., Hirst, A., Treguier, A., Webb, D., 2000. Developments in ocean climate modelling. Ocean Modelling 2, 123-192.

Griffies, S., Pacanowski, R., Hallberg, R., 2000. Spurious diapycnal mixing associated with advection in az-coordinate ocean model. Monthly Weather Review 128, 538-564.

Grue, J., Friss, H.A., Palm, E., Rusas, P.O., 1997. A method for computing unsteady fully nonlinear interfacial waves. J. Fluid Mech. 351, 223-252.

Guo, C., Chen, X., Vlasenko, V., Stashchuk, N., 2011. Numerical investigation of internal solitary waves from the Luzon Strait: Generation process, mechanism and three-dimensional effects. Ocean Modelling 38, 203 - 216.

Hallberg, R., 1995. Some aspects of the circulation in ocean basins with isopycnals intersecting sloping boundaries. Ph.D. thesis. University of Washington.

Hallberg, R., 1997. Stable split time stepping schemes for large-scale ocean modeling. Journal of Computational Physics 135, 54-65. 
Hallberg, R., 2000. Time integration of diapycnal diffusion and Richardson number-dependent mixing in isopycnal coordinate ocean models. Monthly Weather Review 128, 1402-1419.

Hallberg, R., Adcroft, A., 2009. Reconciling estimates of the free surface height in Lagrangian vertical coordinate ocean models with mode-split time stepping. Ocean Modelling 29, 15-26.

Hallberg, R., Rhines, P., 1996. Buoyancy-driven circulation in an ocean basin with isopycnals intersecting the sloping boundary. Journal of Physical Oceanography 26, 913-940.

Heggelund, Y., Vikebø, F., Berntsen, J., Furnes, G., 2004. Hydrostatic and non-hydrostatic studies of gravitational adjustment over a slope. Continental Shelf Research 24, $2133-2148$.

Helfrich, K.R., Melville, W.K., 2006. Long nonlinear internal waves. Annual Review of Fluid Mechanics 38, 395-425.

Higdon, R.L., Bennett, A.F., 1996. Stability analysis of operator splitting for large-scale ocean modeling. Journal of Computational Physics 123, 311 329.

Higdon, R.L., de Szoeke, R.A., 1997. Barotropic-baroclinic time splitting for ocean circulation modeling. Journal of Computational Physics 135, 30 53. 
Hodges, B., Laval, B., Wadzuk, B., 2006. Numerical error assessment and a temporal horizon for internal waves in a hydrostatic model. Ocean Modelling 13, 44-64.

Hofmeister, R., Burchard, H., Beckers, J.M., 2010. Non-uniform adaptive vertical grids for 3D numerical ocean models. Ocean Modelling 33, 70 86.

Horn, D., Imberger, J., Ivey, G., 2001. The degeneration of large-scale interfacial gravity waves in lakes. Journal of Fluid Mechanics 434, 181-207.

Hovmöller, E., 1949. The trough-and-ridge diagram. Tellus 1, 62-66.

Howard, L.N., 1961. Note on a paper of John W. Miles. Journal of Fluid Mechanics 10, 509-512.

Iselin, C., 1939. The influence of vertical and lateral turbulence on the characteristics of the waters at mid-depths. Transactions, American Geophysical Union 20, 414-417.

Jo, T.C., Choi, W., 2002. Dynamics of strongly nonlinear internal solitary waves in shallow water. Studies in Applied Mathematics 109, 205-227.

Jo, T.C., Choi, W., 2008. On stabilizing the strongly nonlinear internal wave model. Studies in Applied Mathematics 120, 65-85.

Kanarska, Y., Maderich, V., 2003. A non-hydrostatic numerical model for calculating free-surface stratified flows. Ocean Dyn. 53, 176-185. 
Kanarska, Y., Shchepetkin, A., McWilliams, J., 2007. Algorithm for nonhydrostatic dynamics in the regional oceanic modeling system. Ocean Modelling 18, $143-174$.

Kang, D., Fringer, O., 2005. Time accuracy of pressure methods for nonhydrostatic free-surface flows, in: Estuarine and Coastal Modeling 2005 - Proceedings of 9th International Conference on Estuarine and Coastal Modeling, ASCE. pp. 419-433.

Kantha, L.H., Clayson, C.A., 2000. Small scale processes in geophysical fluid flows. Academic Press.

Keilegavlen, E., Berntsen, J., 2009. Non-hydrostatic pressure in $\sigma$-coordinate ocean models. Ocean Modelling 28, 240 - 249.

Klingbeil, K., Burchard, H., 2013. Implementation of a direct nonhydrostatic pressure gradient discretisation into a layered ocean model. Ocean Modelling 65, $64-77$.

Klymak, J.M., Legg, S., Pinkel, R., 2010. A simple parameterization of turbulent tidal mixing near supercritical topography. Journal of Physical Oceanography 40.

Klymak, J.M., Legg, S.M., 2010. A simple mixing scheme for models that resolve breaking internal waves. Ocean Modelling 33, 224-234.

Korteweg, D., de-Vries, G., 1895. On the change of form of long waves 
advancing in a rectangular canal, and on a new type of long stationary waves. Philos. Mag. 39, 422-443.

Kramer, S., Cotter, C., Pain, C., 2010. Solving the Poisson equation on small aspect ratio domains using unstructured meshes. Ocean Modelling 35, 253 $-263$.

Kundu, P.K., 1990. Fluid Mechanics. Academic Press.

Lai, Z., Chen, C., Cowles, G., Beardsley, R., 2010a. A nonhydrostatic version of FVCOM: 1. Validation experiments. Journal of Geophysical Research: Oceans 115, n/a-n/a.

Lai, Z., Chen, C., Cowles, G., Beardsley, R., 2010b. A nonhydrostatic version of FVCOM: 2. Mechanistic study of tidally generated nonlinear internal waves in Massachusetts Bay. Journal of Geophysical Research: Oceans $115, \mathrm{n} / \mathrm{a}-\mathrm{n} / \mathrm{a}$.

Leonard, B.P., 1979. A stable and accurate convective modelling procedure based on quadratic upstream interpolation. Comput. Meth. App. Mech. 19, 59-98.

Li, Q., Farmer, D.M., 2011. The generation and evolution of nonlinear internal waves in the deep basin of the South China Sea. Journal of Physical Oceanography 41, 1345 - 1363.

Liu, A.K., 1988. Analysis of nonlinear internal waves in the New York Bight. J. Geophys. Res. 93, 12317-12329. 
Liu, P.L.F., Wang, X., 2012. A multi-layer model for nonlinear internal wave propagation in shallow water. Journal of Fluid Mechanics 695, 341-365.

Llewellyn Smith, S., Young, W., 2002. Conversion of the barotropic tide. Journal of Physical Oceanography 32, 1554-1566.

Lynett, P., Liu, P., 2002. A two-dimensional, depth-integrated model for internal wave propagation over variable bathymetry. Wave Motion 36, 221 $-240$.

Mahadevan, A., Oliger, J., Street, R., 1996a. A nonhydrostatic mesoscale ocean model. Part I: Implementation and scaling. J. Phys. Oceanogr. 26, $1860-1879$.

Mahadevan, A., Oliger, J., Street, R., 1996b. A nonhydrostatic mesoscale ocean model. Part II: Numerical implementation. J. Phys. Oceanogr. 26, $1880-1900$.

Mandli, K.T., 2013. A numerical method for the two layer shallow water equations with dry states. Ocean Modelling 72, 80 - 91.

Marshall, J., Adcroft, A., Hill, C., Perelman, L., Heisey, C., 1997a. A finitevolume, incompressible Navier-Stokes model for studies of the ocean on parallel computers. J. Geophys. Res. 102, 5753-5766.

Marshall, J., Hill, C., Perelman, L., Adcroft, A., 1997b. Hydrostatic, quasihydrostatic, and nonhydrostatic ocean modeling. J. Geophys. Res. 102, $5733-5752$. 
Miao, C., Chen, H., Lü, X., 2011. An isopycnic-coordinate internal tide model and its application to the South China Sea. Chinese Journal of Oceanology and Limnology 29, 1339-1356.

Miles, J.W., 1961. On the stability of heterogeneous shear flows. J. Fluid Mech 10, 496-508.

Montgomery, R., 1940. The present evidence on the importance of lateral mixing processes in the ocean. Bull. Amer. Meteor. Soc 21, 87-94.

Müller, P., 2006. The equations of oceanic motions. Cambridge University Press.

Redi, M.H., 1982. Oceanic isopycnal mixing by coordinate rotation. Journal of Physical Oceanography 12, 1154-1158.

Schopf, P., Loughe, A., 1995. A reduced-gravity isopycnal ocean model: Hindcasts of El Niño. Monthly weather review 123, 2839-2863.

Scotti, A., Beardsley, R., Butman, B., Pineda, J., 2008. Shoaling of nonlinear internal waves in Massachusetts Bay. Journal of Geophysical Research: Oceans (1978-2012) 113.

Scotti, A., Beardsley, R.C., Butman, B., 2007. Generation and propagation of nonlinear internal waves in Massachusetts Bay. Journal of Geophysical Research: Oceans 112, n/a-n/a. 
Scotti, A., Mitran, S., 2008. An approximated method for the solution of elliptic problems in thin domains: Application to nonlinear internal waves. Ocean Modelling 25, 144-153.

Simmons, H., Chang, M., Chang, Y., Chao, S., Fringer, O., Jackson, C., Ko, D., 2011. Modeling and prediction of internal waves in the South China Sea. Oceanography-Oceanography Society 24, 88.

Simmons, H.L., Hallberg, R.W., Arbic, B.K., 2004. Internal wave generation in a global baroclinic tide model. Deep Sea Research Part II: Topical Studies in Oceanography 51, $3043-3068$.

Smolarkiewicz, P.K., Margolin, L.G., 1998. MPDATA: A finite-difference solver for geophysical flows. Journal of Computational Physics 140, 459 480.

Solomon, H., 1971. On the representation of isentropic mixing in ocean circulation models. Journal of Physical Oceanography 1, 233-234.

Steinmoeller, D., Stastna, M., Lamb, K., 2012. Fourier pseudospectral methods for 2d Boussinesq-type equations. Ocean Modelling 52-53, 76 - 89.

Stelling, G., Zijlema, M., 2003. An accurate and efficient finite-difference algorithm for non-hydrostatic free-surface flow with application to wave propagation. International Journal for Numerical Methods in Fluids 43, $1-23$. 
Stelling, G.S., Duinmeijer, S.P.A., 2003. A staggered conservative scheme for every froude number in rapidly varied shallow water flows. International Journal for Numerical Methods in Fluids 43, 1329-1354.

Vitousek, S., Fringer, O.B., 2011. Physical vs. numerical dispersion in nonhydrostatic ocean modeling. Ocean Modelling 40, 72 - 86 .

Vitousek, S., Fringer, O.B., 2013. Stability and consistency of nonhydrostatic free-surface models using the semi-implicit $\theta$-method. International Journal for Numerical Methods in Fluids 72, 550-582.

Vlasenko, V., Sanchez Garrido, J.C., Stashchuk, N., Garcia Lafuente, J., Losada, M., 2009. Three-dimensional evolution of large-amplitude internal waves in the Strait of Gibraltar. Journal of Physical Oceanography 39, $2230-2246$.

Vlasenko, V., Stashchuk, N., 2007. Three-dimensional shoaling of largeamplitude internal waves. Journal of Geophysical Research: Oceans 112, $\mathrm{n} / \mathrm{a}-\mathrm{n} / \mathrm{a}$.

Vlasenko, V., Stashchuk, N., Guo, C., Chen, X., 2010. Multimodal structure of baroclinic tides in the South China Sea. Nonlinear Processes in Geophysics 17, 529-543.

Warn-Varnas, A., Hawkins, J., Lamb, K., Piacsek, S., Chin-Bing, S., King, D., Burgos, G., 2010. Solitary wave generation dynamics at Luzon Strait. Ocean Modelling 31, 9-27. 
White, A., Bromley, R., 1995. Dynamically consistent, quasi-hydrostatic equations for global models with a complete representation of the Coriolis force. Quarterly Journal of the Royal Meteorological Society 121, 399-418.

Willebrand, J., Barnier, B., Bning, C., Dieterich, C., Killworth, P.D., Le Provost, C., Jia, Y., Molines, J.M., New, A.L., 2001. Circulation characteristics in three eddy-permitting models of the North Atlantic. Progress in Oceanography 48, $123-161$.

Zhang, Z., Fringer, O.B., Ramp, S.R., 2011. Three-dimensional, nonhydrostatic numerical simulation of nonlinear internal wave generation and propagation in the South China Sea. J. Geophys. Res. 116.

Zijlema, M., Stelling, G.S., 2005. Further experiences with computing nonhydrostatic free-surface flows involving water waves. International Journal for Numerical Methods in Fluids 48, 169-197. 